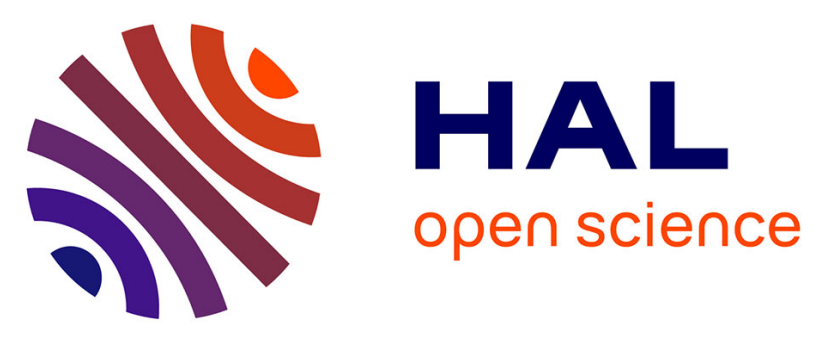

\title{
On 3D DDFV discretization of gradient and divergence operators. II. Discrete functional analysis tools and applications to degenerate parabolic problems.
}

Boris Andreianov, Mostafa Bendahmane, Florence Hubert

\section{- To cite this version:}

Boris Andreianov, Mostafa Bendahmane, Florence Hubert. On 3D DDFV discretization of gradient and divergence operators. II. Discrete functional analysis tools and applications to degenerate parabolic problems.. Computational Methods in Applied Mathematics, 2013, 13 (4), pp. 369-410. 10.1515/cmam-2013-0011 . hal-00567342v3

\section{HAL Id: hal-00567342 \\ https://hal.science/hal-00567342v3}

Submitted on 22 Apr 2013

HAL is a multi-disciplinary open access archive for the deposit and dissemination of scientific research documents, whether they are published or not. The documents may come from teaching and research institutions in France or abroad, or from public or private research centers.
L'archive ouverte pluridisciplinaire HAL, est destinée au dépôt et à la diffusion de documents scientifiques de niveau recherche, publiés ou non, émanant des établissements d'enseignement et de recherche français ou étrangers, des laboratoires publics ou privés.

\section{(ㄷ)(1) $\$$}

Distributed under a Creative Commons Attribution - NonCommerciall 4.0 International 


\title{
On 3D DDFV discretization of gradient and divergence operators. II. Discrete functional analysis tools and applications to degenerate parabolic problems.
}

\author{
B. Andreianov • M. Bendahmane • F. Hubert
}

\begin{abstract}
We present a detailed survey of discrete functional analysis tools (consistency results, Poincaré and Sobolev embedding inequalities, discrete $W^{1, p}$ compactness, discrete compactness in space and in time) for the so-called Discrete Duality (DDFV) Finite Volume schemes in three space dimensions. We concentrate mainly on the 3D CeVe-DDFV scheme presented in [3]. Some of our results are new, such as a general time-compactness result based upon the idea of Kruzhkov [65]; others generalize the ideas known for the 2D DDFV schemes or for traditional two-point finite volume schemes.

We illustrate the use of these tools by studying convergence of discretizations of nonlinear elliptic-parabolic problems of Leray-Lions kind, and provide numerical results for this example.
\end{abstract}

2010 Mathematical subject classification: 65N12; 65M12.

Keywords: finite volume approximation; discrete duality; 3D CeVe-DDFV; convergence; consistency; discrete compactness; Kruzhkov time compactness lemma; discrete Sobolev embeddings; degenerate parabolic problems.

\section{Introduction}

Duality formula linking discrete divergence and discrete gradient operators is a key for proving convergence of many known numerical schemes for elliptic and parabolic PDEs. The name of DDFV (Discrete Duality Finite Volume) was given to a particular kind of 2D schemes that possess this duality feature and work on "double" meshes (see Hermeline $[57,60]$ and references therein; Domelevo and Omnès [36]; cf. Nicolaïdes [69]). The importance of the discrete duality feature stems from the fact that it makes finite volume discretizations of numerous elliptic operators "structure-preserving" (which means e.g. that the discretization of monotone coercive operators is monotone and coercive). The other

Boris Andreianov

Laboratoire de Mathématiques de Besançon CNRS UMR 6623, Université de Franche-Comté,

16 route de Gray, 25030 Besançon, France

E-mail: boris.andreianov@univ-fcomte.fr.

Mostafa Bendahmane

Institut de Mathématiques de Bordeaux, Université Victor Segalen, 33076 Bordeaux Cedex, France

E-mail: mostafa.bendahmane@u-bordeaux2.fr.

Florence Hubert

LATP, Université de Provence, 39 rue F. Joliot-Curie, 13453 Marseille Cedex 13, France

E-mail: fhubert@cmi.univ-mrs.fr. 
important feature is that DDFV schemes can be used for anisotropic elliptic operators on almost general meshes, and for nonlinear elliptic problems. Many other types of schemes are able to cope with the same difficulties: we refer to $[55,45]$ for an extensive benchmark comparison in 2D and in 3D. For different applications of DDFV schemes, we refer to $[4,7,12,19,20,21,23,25,26,28,30,31,34,35,36,57,58,60,61,62,63,64,68,70,72]$. A priori error estimates for were obtained, e.g., in [36, 12, 20], and a posteriori estimates were recently derived in [67] for the case of the Stokes problem.

The main goal of this paper is to survey several tools for analysis of DDFV schemes and to demonstrate their usefulness by giving a convergence proof for one particular application (see also [36, 12, 20, 4, 7, 26, 66] for this purpose). To be specific, we study convergence for degenerate elliptic-parabolic equations and illustrate the convergence theorem by numerical experiments (see [14] for other tests). The present paper partially follows the guidelines of Andreianov, Boyer and Hubert [12], where the 2D DDFV method was used for discretization of nonlinear diffusion problems. But we focus on the 3D DDFV schemes from the works $[5,3,14]$ of the authors and Karlsen and Krell. In these works, a 3D generalization of the 2D DDFV scheme, called CeVe-DDFV, was described and the discrete duality property was justified. The 3D CeVe-DDFV construction coincides with the one given by Hermeline in $[60]^{1}$. Note that other kinds of 3D DDFV schemes are in use. First, a slightly different CeVeDDFV scheme was designed by Pierre in [72] (see also [32, 30, 31] and [29]). Developing the approach of Hermeline [59] with introduction of additional unknowns, Coudière and Hubert proposed a 3D scheme with a different idea for mesh and gradient construction, now called CeVeFE-DDFV: see [25, 26] and [27]. Note that the discrete functional analysis tools of this paper easily extend to 3D CeVeFE-DDFV schemes. A 3D DDFV construction inspired by [26] was recently proposed in [46]; this construction boils down to a generalization of the scheme of [72]. More information is given in [3]. Many other methods for linear anisotropic, or nonlinear, elliptic problems on general meshes have been proposed in the last decade; we refer to the survey paper [38] and the references given in [3, 38]. An extended numerical comparison of different schemes on linear 3D diffusion problems was carried out in the benchmark [45], see in particular [14, 27, 29] for different kinds of 3D DDFV schemes. For related numerical results for various 3D DDFV schemes, we refer to Hermeline [59, 60] and Coudière and Hubert [25] (in the context of linear heterogeneous anisotropic diffusion operators), to Coudière et al. [30, 32, 31] (in the context of electrocardiology, cf. [16, 17] and $[7])$.

Let us give an outline of the paper. In Section 2 we recall the basic notation for 3D DDFV schemes, spaces of discrete functions and fields, discrete divergence and discrete gradient operators; the details are given in [3]. This notation reflects the far-reaching analogy between the continuous framework and the discrete DDFV framework, and makes the convergence proofs quite similar to the proofs of structural stability (i.e., of the stability of solutions with respect to perturbation of data and nonlinearities) in the continuous framework, cf. [6].

In Section 3, we first give consistency results for the mesh projection operators and for the discrete gradient operator. Then, we discuss the discrete Poincaré and Sobolev embeddings for the DDFV schemes (cf. [12] and [22] for the 2D case), and sketch the proof of the embeddings in the case of the Neumann boundary conditions. Then we give two kinds of discrete compactness results. The principle of $W^{1, p}$ weak compactness in space is well

\footnotetext{
${ }^{1}$ The work [60] imposes unnecessary restrictions on the meshes but it treats the more delicate case of diffusion operators with discontinuous coefficients.
} 
known (see in particular [12] for the 2D case). The $L^{1}$ space-time compactness is a new result of independent interest: its proof is based upon the original idea of Kruzhkov [65]. We refer to $[49,51,2]$ and references therein for different approaches to time compactness. Two important technical issues are treated in appendices. In Appendix A, we introduce a penalization operator, helpful in some convergence studies, and in Appendix B we give a hint on discretization of reaction terms in the context of DDFV schemes.

As an application, we discuss several particular cases of the general triply nonlinear elliptic-parabolic-hyperbolic equation (see [6] and references therein)

$$
b(u)_{t}+\operatorname{div} F(u)=\operatorname{div} \varphi(\nabla A(u))+f,
$$

where $b(\cdot), A(\cdot)$ are continuous non-strictly increasing functions, $F(\cdot)$ is continuous and $\varphi(\cdot)$ is a nonlinearity of Leray-Lions type. The $p$-laplacian operator, i.e., the case of $\varphi(\xi)=|\xi|^{p-2} \xi$, is the main example in the applications; more generally, it could be combined with an anisotropy and heterogeneity, as for the case $\varphi(\xi, x)=|A(x) \xi|^{p-2} A(x) \xi,(A(x))_{x \in \Omega}$ being a uniformly elliptic bounded family of symmetric matrices. In Section 4.1 we give the proof of convergence of our 3D DDFV discretizations ${ }^{2}$ for the elliptic-parabolic case $(A=\mathrm{Id}$, $F=0$ ) with the homogeneous Dirichlet boundary conditions. We refer to [13] and references therein for previous results on finite volume approximation of elliptic-parabolic problems; this encompasses the Richards equation used to model one-phase flows in porous media. Further, in Section 4.2 we briefly discuss the additional tools needed to treat the case of hyperbolic degeneracy in (1) ([4]). One more aspect of problem (1) is exposed in the work $[15]$.

In Section 5, we report on numerical results for the problem treated in Section 4.1. Recall that the problem we solve numerically is a nonlinear one; Appendix C presents the coordination-decomposition iterative algorithm (see $[53,54,20])$ used in the numerical tests of the present paper.

\section{Notation for the scheme, operators and discrete duality}

Let us briefly recall our discrete framework, limited to the case of operators with homogeneous Dirichlet or Neumann boundary conditions. The cases of non-homogeneous and mixed boundary conditions are treated in [12] and in [7].

We only reproduce the notation needed to understand the statements of the paper and the applications in Section 4. Illustrative figures and additional notation that is only needed in some proofs of Section 3 are given without comment in Section 2.2 (for details, see the paper [3] of the authors and S. Krell).

DDFV schemes are designed for discretization in space of second-order elliptic operators in divergence form; therefore we concentrate on description of the meshes of a domain $\Omega \subset \mathbb{R}^{3}$ (notation related to the time dependence is given in Section 3.5). The 3D CeVe-DDFV meshes of $\Omega$ described in [3, 14] (cf. [5] and Hermeline [60]) are triples $\mathfrak{T}=\left(\overline{\mathfrak{M}^{\mathfrak{o}}}, \overline{\mathfrak{M}^{*}}, \mathfrak{D}\right)$; actually both $\overline{\mathfrak{M}^{*}}$ and $\mathfrak{D}$ are constructed from $\overline{\mathfrak{M}^{o}}$ which is given.

\footnotetext{
${ }^{2}$ Note that we add the penalization operator to the scheme in order to ensure convergence, as explained in Section 3.4. Numerically, the non-penalized scheme converges as well. Proving convergence of the nonpenalized scheme is beyond the scope of this paper, but this question deserves further study.
} 
The mesh $\boldsymbol{T}$ consists of control volumes of two kinds, the primal ones (denoted $k \in \overline{\mathfrak{M}^{\mathfrak{o}}}$ ) and the dual ones (denoted $K^{*} \in \overline{\mathfrak{M}^{*}}$ ). Both primal and dual volumes form a partition of $\Omega$, up to a set of measure zero. The volumes are associated with centers $x_{K}$ and $x_{K^{*}}$, respectively; one may assume that $x_{K} \in K$ and $x_{K^{*}} \in K^{*}$. The dual volumes are constructed using, as vertices, the primal volume centers $\left(x_{K}\right)_{K}$, some face centers $\left(x_{K \mid L}\right)_{K \mid L}$ (K|L being the notation for a face separating neighbors $K$ and $L$ ) and the edge middlepoints $\left(x_{K^{*} \mid L^{*}}\right)_{K^{*} L^{*}}$ ( $K^{*}$ and $L^{*}$ being neighbor dual centers). For details, we refer to [3, Sec. 2] (see also [60]); important generalizations can be found in [3, Sec. 4].

The dual and primal volumes with centers lying on $\partial \Omega$ are considered as "boundary" volumes (denoted $K \in \partial \mathfrak{M}^{\circ}$ and $K^{*} \in \partial \mathfrak{M}^{*}$, resp.), in the case we look at Dirichlet boundary conditions. The primal boundary volumes are in fact fictive ones (they are flat: each face of $K \in \mathfrak{M}^{o}$ contained in $\partial \Omega$ gives rise to a boundary volume). With each (primal or dual) interior control volume (denoted $K \in \mathfrak{M}^{o}$ or $K^{*} \in \mathfrak{M}^{*}$, resp.), unknown values $u_{K}$, resp. $u_{K^{*}}$ for a discrete solution $u$ are associated (the value $u_{K}$, resp. $u_{K^{*}}$, is seen as the value of $u$ at the point $x_{K}$, resp. $x_{K^{*}}$ ); Dirichlet boundary conditions are imposed at the centers of the boundary volumes. The Neumann or Robin boundary conditions, when present, enter the definition of the discrete divergence operator (see $[21,62,23]$ and [7] for details). In the application, we will focus on the homogeneous Dirichlet condition.

Thus we consider the space $\mathbb{R}^{\mathfrak{T}}$ of discrete functions on interior volumes; a discrete function $u^{\mathfrak{T}} \in \mathbb{R}^{\mathfrak{T}}$ consists of one real value per primal or dual interior control volume. The space of discrete functions that, in addition, take the value zero in the boundary volumes is denoted by $\mathbb{R}_{0}^{\overline{\mathfrak{T}}}$; more generally, by $u^{\overline{\mathfrak{T}}} \in \mathbb{R}^{\overline{\mathfrak{T}}}$ we mean an extension of $u^{\mathfrak{T}} \in \mathbb{R}^{\mathfrak{T}}$ by values assigned in boundary volumes. On $\mathbb{R}^{\mathfrak{T}}$, we consider the appropriate scalar product $\llbracket[\cdot, \cdot]_{\Omega}$ (see formula (3) below).

A third mesh consisting of diamond volumes (denoted $D \in \mathfrak{D}$ ) is required to handle discrete gradients and other discrete fields on $\Omega$. A diamond $D$ is uniquely determined by a couple $K, L$ of primal volumes that have common interface $K \mid L$ contained within $D$; in this case, we denote it $D^{K L L}$. If $x_{K} \in K, x_{L} \in L$ and $K \mid L$ is convex, then the diamond $D^{K \mid L}$ is the union of convex hulls of $x_{K}, K \mid L$ and of $x_{L}, K \mid L$ (see [3, Sec.4] for several generalizations) ${ }^{3}$. The space $\left(\mathbb{R}^{\mathfrak{D}}\right)^{3}$ of discrete fields on $\Omega$ serves to define the fluxes through the boundaries of control volumes. A discrete field $\overrightarrow{\mathcal{F}}^{\mathfrak{T}} \in\left(\mathbb{R}^{\mathfrak{D}}\right)^{3}$ on $\Omega$ consists of one $\mathbb{R}^{3}$ value per diamond. On $\left(\mathbb{R}^{\mathfrak{D}}\right)^{3}$, one considers the appropriate scalar product $\{\{\cdot, \cdot\}\}_{\Omega}$ (see formula (4) below).

A Discrete Duality Finite Volume scheme is determined by the mesh, the discrete divergence operator $\operatorname{div}^{\mathfrak{T}}:\left(\mathbb{R}^{\mathfrak{D}}\right)^{3} \longrightarrow \mathbb{R}^{\mathfrak{T}}$ (see formula (5) below) obtained by the standard finite volume discretization procedure (taking into account the Neumann or Robin boundary condition, if necessary), and by the associated discrete gradient operator $\nabla^{\mathfrak{T}}: \mathbb{R}^{\mathfrak{T}} \longrightarrow\left(\mathbb{R}^{\mathfrak{D}}\right)^{3}$ (see formula (7) below) obtained diamond-wise, by a kind of affine interpolation (see [3] for the motivation behind the definition of $\nabla^{\mathfrak{T}}$ ). The essential property of the DDFV schemes is the discrete duality [3, Prop.3.2] (see also the proof of [60, Th.1]), stated as

$$
\left[\left[-\operatorname{div}^{\mathfrak{T}} \overrightarrow{\mathcal{F}}^{\mathfrak{T}}, v^{\mathfrak{T}}\right]\right]_{\Omega}=\left\{\left\{\overrightarrow{\mathcal{F}}^{\mathfrak{T}}, \nabla^{\mathfrak{T}} v^{\overline{\mathfrak{T}}}\right\}\right\}_{\Omega} ;
$$

\footnotetext{
${ }^{3}$ Diamonds are further cut into subdiamonds, denoted $S_{K^{*} L^{*}}^{K L}$ (or $S \in \mathfrak{S}$, for a generic subdiamond) that involve neighbors $K, L$ and dual neighbors $K^{*}, L^{*}$ intersecting $K, L$ (see Fig. 2); these are only needed inside some proofs of Section 3.
} 
this form is suitable either for $v$ satisfying the homogeneous Dirichlet boundary condition, or for $\overrightarrow{\mathcal{F}}$ satisfying the homogeneous Neumann (zero-flux) boundary condition (see [7, 62] for the general case including a boundary scalar product).

The discrete solution $u^{\mathfrak{z}}$ will be often identified with the piecewise constant function

$$
u^{\mathfrak{T}}:=\frac{1}{3} v^{\mathfrak{m}^{o}}+\frac{2}{3} v^{\mathfrak{M}^{*}}
$$

with $v^{\mathfrak{m}^{o}}$ and $v^{\mathfrak{m}^{*}}$ representing the piecewise constant discrete solutions on the primal and the dual mesh, respectively; e.g., $v^{\mathfrak{M}^{o}}(x)=\sum_{K \in \mathfrak{M}^{\circ}} v_{K} 1_{K}(x)$. Similarly, we identify $\nabla^{\mathfrak{T}} u^{\overline{\mathfrak{T}}}$ with the piecewise constant function $\nabla^{\mathfrak{T}} u^{\overline{\mathfrak{s}}}:=\sum_{D \in \mathfrak{D}} \nabla_{D} u^{\overline{\mathfrak{s}}} 1_{D}(x)$. This identification is related to the notion of reconstruction (lifting) operator used in the context of "gradient schemes", see Eymard, Guichard and Herbin [46].

We denote by $\operatorname{Vol}(A)$ the three-dimensional Lebesgue measure of $A$ which can stand for a control volume, a dual control volume, a diamond, etc.

\subsection{An illustration: Cartesian DDFV meshes in 3D}

As an illustration, let us reproduce the simple example of a 3D DDFV mesh given in [3, Sect. 2.3]. Take the unit cube $\Omega=[0,1]^{3}$ and partition it into $N^{3}$ primal cubic volumes of edge $\frac{1}{N}$. The diamonds are octahedra built on two primal cubes' centers $x_{K_{\odot}}, x_{K_{\oplus}}$ and on the square interface $K_{\odot} \mid K_{\oplus}$ between them. One chooses for $x_{K_{\odot} \mid K_{\oplus}}$ the center of symmetry of $K_{\odot} \mid K_{\oplus}$. The interior dual volumes are also cubes of the same edge $\frac{1}{N}$ centered at the vertices of the primal mesh that do not lie on $\partial \Omega$. Fig. 1 pictures the primal and dual mesh and one diamond.

On uniform cubic mesh, a notation simpler than that of the general case can be used; in particular, discrete gradient and divergence operators can be computed by evident formulas given in [3, Sect.2.3]. We will prove several results specific to these meshes. Notice that the DDFV approach on Cartesian meshes can be directly compared to the approach of [9] (developed in 2D) ${ }^{4}$. While this kind of schemes looks too complicated for linear problems on Cartesian meshes, it can be useful e.g. for image processing applications involving 1Laplacian kind diffusion operators (see [61]; cf. [48] and references therein).

\subsection{Notation and formulas used in some proofs}

For $w^{\mathfrak{T}}, v^{\mathfrak{T}} \in \mathbb{R}^{\mathfrak{T}}$ and for $\overrightarrow{\mathcal{F}}^{\mathfrak{T}}, \overrightarrow{\mathcal{G}}^{\mathfrak{T}} \in\left(\mathbb{R}^{3}\right)^{\mathfrak{P}}$, the scalar products are given by

$$
\begin{aligned}
\left.\llbracket\left[w^{\mathfrak{z}}, v^{\mathfrak{T}}\right]\right]_{\Omega}= & \frac{1}{3} \sum_{K \in \mathfrak{M}^{o}} \operatorname{Vol}(K) w_{K} v_{K}+\frac{2}{3} \sum_{K^{*} \in \mathfrak{M}^{*}} \operatorname{Vol}\left(K^{*}\right) w_{K^{*}} v_{K^{*}} ; \\
& \left\{\left\{\overrightarrow{\mathcal{F}}^{\mathfrak{T}}, \overrightarrow{\mathcal{G}}^{\mathfrak{z}}\right\}_{\Omega}=\sum_{D \in \mathfrak{D}} \operatorname{Vol}(D) \overrightarrow{\mathcal{F}}_{D} \cdot \overrightarrow{\mathcal{G}}_{D} .\right.
\end{aligned}
$$

The entries of the discrete divergence of a field $\mathcal{F}$ can be computed by the formulas

$$
\begin{aligned}
\operatorname{div}_{K} \overrightarrow{\mathcal{F}}^{\mathfrak{T}} & =\frac{1}{2 \operatorname{Vol}(K)} \sum_{\mathrm{S} \sim K}\left(-1 \epsilon^{\epsilon_{S}^{K}}\left\langle\overrightarrow{\mathcal{F}}_{\mathrm{S}}, \overrightarrow{x_{\odot, \oplus}^{*} x_{i, i+1}^{*}}, \overrightarrow{x_{i}^{*} x_{i+1}^{*}}\right\rangle,\right. \\
\operatorname{div}_{K^{*}} \overrightarrow{\mathcal{F}}^{\mathfrak{T}} & =\frac{1}{2 \operatorname{Vol}\left(K^{*}\right)} \sum_{\mathrm{S} \sim K^{*}}(-1)^{\epsilon_{S}^{*}}\left\langle\overrightarrow{\mathcal{F}}_{\mathrm{S}}, \overrightarrow{x_{\odot} x_{\oplus}}, \overrightarrow{x_{\odot, \oplus}^{*} x_{i, i+1}^{*}}\right\rangle .
\end{aligned}
$$

\footnotetext{
${ }^{4}$ In [9] and the subsequent works [10,11], the vector $\nabla_{D} u^{\mathfrak{T}}$ was reconstructed per quarter of diamond and its absolute value was reconstructed per diamond by averaging of the quarter-diamonds.
} 
(see the notation of Fig. 2). In formulas (5), we mean that each subdiamond $S$ associated with $K$ (resp., with $K^{*}$ ) has the form $S=S_{K_{i}^{*} \mid K_{i+1}^{*}}$, with some $K_{\odot}, K_{\oplus}, K_{i}^{*}, K_{i+1}^{*}$; the notation under the sign " $\sum$ " refers to $S=S_{K_{i}^{*} \mid K_{i+1}^{*}}^{K_{\odot} \mid K_{\oplus}}$. Because $K$ may coincide either with $K_{\odot}$ or with $K_{\oplus}$ (similarly, $K^{*}$ may be $K_{i}^{*}$ or $K_{i+1}^{*}$ ), the "sign selectors"

$$
\epsilon_{\mathrm{S}}^{K}:=\left\{\begin{array}{l}
0, \text { if } K=K_{\odot} \\
1, \text { if } K=K_{\oplus}
\end{array}, \quad \epsilon_{\mathrm{S}}^{K^{*}}:=\left\{\begin{array}{l}
0, \text { if } K^{*}=K_{i}^{*} \\
1, \text { if } K^{*}=K_{i+1}^{*}
\end{array}\right.\right.
$$

are introduced in (5). The meaning of formulas (5) is standard in the context of finite volume schemes: e.g., $\operatorname{Vol}(K) \operatorname{div}_{K} \overrightarrow{\mathcal{F}}$ expresses the boundary flux $\int_{\partial K} \overrightarrow{\mathcal{F}} \cdot \overrightarrow{\nu_{K}}$ (see $[3$, Lemma $3.1])$.

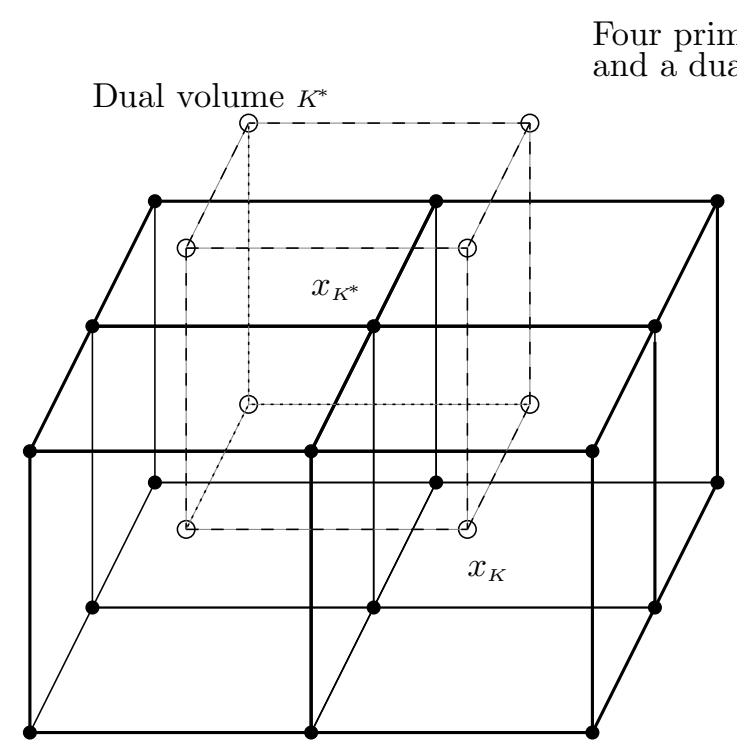

Primal volume $K$

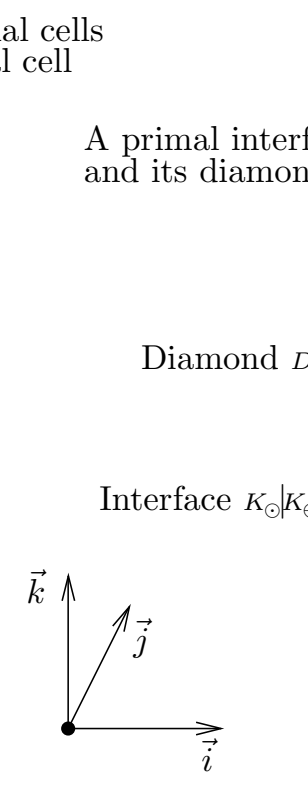

A primal interface and its diamond
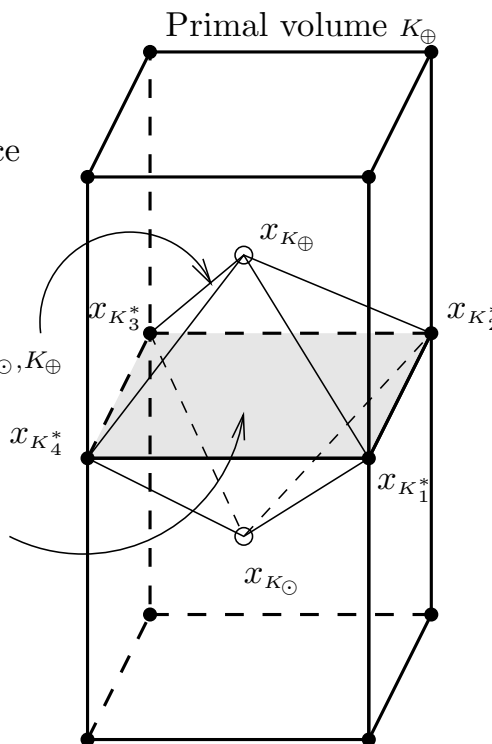

Primal volume $K_{\odot}$

Figure 1. Example of Cartesian DDFV mesh in 3D (left). Two neighbour volumes and a diamond (right).
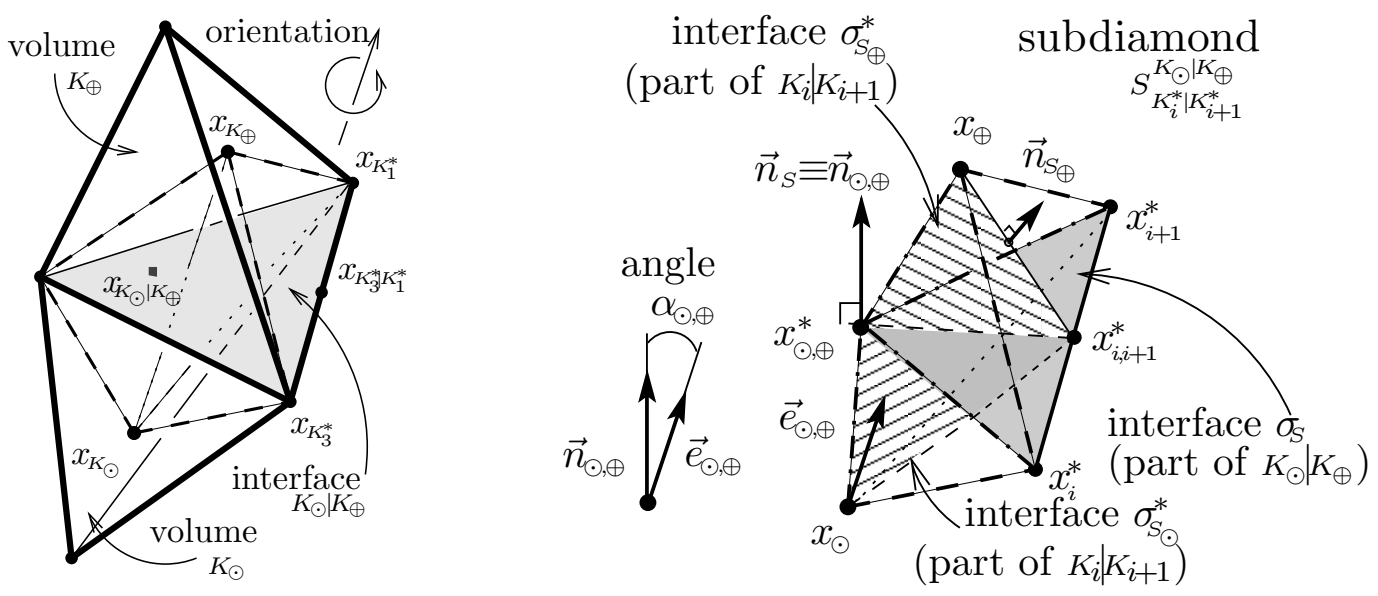

Figure 2. $3 \mathrm{D}$ neighbor volumes and the associated diamond $D^{K_{\odot}} K_{\oplus}$ (left). Zoom on a subdiamond (right). 
Similarly, the entries of the discrete gradient are calculated by the formula

$$
\nabla_{D} w^{\overline{\mathfrak{s}}}=\frac{1}{6 \operatorname{Vol}(D)} \sum_{i=1}^{l}\left\{\frac{\left\langle\overrightarrow{x_{\odot} x_{\oplus}}, \overrightarrow{x_{\odot, \oplus}^{*} x_{i, i+1}^{*}}, \overrightarrow{x_{i}^{*} x_{i+1}^{*}}\right\rangle}{\overrightarrow{x_{\odot} x_{\oplus}} \cdot \vec{n}_{\odot, \oplus}}\left(w_{\oplus}-w_{\odot}\right) \vec{n}_{\odot, \oplus}+2\left(w_{i+1}^{*}-w_{i}^{*}\right)\left[\overrightarrow{x_{\odot} x_{\oplus}} \times \overrightarrow{x_{\odot \oplus \oplus}^{*} x_{i, i+1}^{*}}\right]\right\}
$$

with the notation of Fig. 2 (the depicted case corresponds to primal interface $K_{\odot} \mid K_{\oplus}$ with $l=3$ vertices, with the convention " $l+1:=1$ "); in $(7), \cdot \times \cdot$ and $\langle\cdot, \cdot, \cdot\rangle$ denote the vector and the mixed products of vectors of $\mathbb{R}^{3}$, respectively. Let us explain the meaning of (7). The component of $\nabla_{D} w^{\overline{\mathfrak{s}}}$ along the direction $\overrightarrow{x_{\odot} x_{\oplus}}$ is denoted by $\operatorname{Proj}_{D}\left(\nabla_{D} w^{\overline{\mathfrak{s}}}\right)$, and $\operatorname{Proj}_{D}^{*}\left(\nabla_{D} w^{\overline{\mathfrak{s}}}\right)$ is used for the component along the face $K \mid L$; then setting $V=\sum_{i=1}^{l}\left\langle\vec{n}_{\odot, \oplus}, \overrightarrow{x_{\odot, \oplus}^{*} x_{i, i+1}^{*}}, \overrightarrow{x_{i}^{*} x_{i+1}^{*}}\right\rangle$ we have (see [3, Lemma 2.4])

$$
\nabla_{D} w^{\overline{\mathfrak{s}}} \text { is s.t. }\left\{\begin{array}{l}
\operatorname{Proj}_{D}\left(\nabla_{D} w^{\overline{\mathfrak{s}}}\right)=\frac{w_{\oplus}-w_{\odot}}{d_{\odot, \oplus}} \vec{e}_{\odot, \oplus}, \\
\operatorname{Proj}_{D}^{*}\left(\nabla_{D} w^{\overline{\mathfrak{s}}}\right)=\frac{2}{V} \sum_{i=1}^{l}\left(w_{i+1}^{*}-w_{i}^{*}\right)\left[\vec{n}_{\odot, \oplus} \times \overline{x_{\odot, \oplus}^{*} x_{i, i+1}^{*}}\right]
\end{array}\right.
$$

Let us stress that the discrete gradient is consistent with affine functions, see $[3$, Prop. 2.3]:

\section{Proposition 2.1 (Consistency of the discrete gradient).}

Let $w_{\odot}, w_{\oplus},\left(w_{i}^{*}\right)_{i=1}^{l}$ be the values at the points $x_{\odot}, x_{\oplus},\left(x_{i}\right)_{i=1}^{l}$, respectively, of an affine on $D=D^{K_{\odot} \mid K_{\oplus}}$ function $w$. Then $\nabla_{D} w^{\bar{\Sigma}}$ coincides with the value of $\nabla w$ on $D$.

\section{Discrete functional analysis tools}

In this section, we give a few "discrete functional analysis tools" related to CeVe-DDFV schemes (such properties or their analogues also hold for 2D DDFV schemes and for 3D CeVeFE-DDFV schemes). Notice that the proof of the asymptotic discrete compactness property of Proposition 3.4 is based upon the discrete duality; the duality is also used, in a much weaker form, in the proof of Proposition 3.5. This section is essentially self-contained; some details are given in references $[8,12,42]$, notation is taken from Section 2.2.In addition, two hints on DDFV discretization (namely, the penalization operator and a structurepreserving discretization of the reaction terms) that may be useful for coping with "double" nature of the CeVe-DDFV approximations are postponed to Appendices A and B.

For a given CeVe-DDFV mesh $\mathfrak{T}$ of $\Omega$ the size of $\mathfrak{T}$ is defined as

$\operatorname{size}(\mathfrak{T}):=\max \left\{\max _{K \in \overline{\mathfrak{M}^{o}}} \operatorname{diam}\left(K \cup\left\{x_{K}\right\}\right), \max _{K^{*} \in \overline{\mathfrak{M}^{*}}} \operatorname{diam}\left(K^{*} \cup\left\{x_{K^{*}}\right\}\right), \max _{D \in \mathfrak{D}} \operatorname{diam}(D)\right\}$.

Throughout this paper, we assume that the mesh satisfies one of the three following assumptions. When discrete Poincaré-kind inequalities are not needed, we assume that

$\boldsymbol{T}$ is a general 3D CeVe-DDFV mesh of the kind described in Section 2

(see [3, Sect. 2 and 4] for more details on the construction of the mesh).

When Poincaré-kind inequalities are essential, we assume either that

in addition to $(M)$, all the faces of primal volumes of $\mathfrak{T}$ are triangles, 
or that

the mesh $\mathfrak{T}$ is the cartesian mesh described in Section 2.1.

In many results, we will work with a family of meshes such that $\operatorname{size}(\boldsymbol{T})$ goes to zero.

\subsection{Regularity assumptions on the meshes}

In various finite volume methods, one always needs some qualitative restrictions on each mesh $\boldsymbol{T}$ considered (such as, e.g., the assumption that $x_{K} \in K$, or the convexity of volumes and/or diamonds, or the mesh orthogonality, or the Delaunay condition on a simplicial mesh). For the convergence analysis on families of such meshes, it is convenient (though not always necessary) to impose shape regularity assumptions on the family of meshes considered. These assumptions are quantitative: this means that the "distortion" of certain objects in a mesh is measured with the help of a regularity constant $\operatorname{reg}(\boldsymbol{T})$, which is finite for each individual mesh but may get unbounded if an infinite family of meshes is considered.

For the 3D DDFV meshes presented in this paper, there are two main mesh regularity assumptions. First, we require several lower bounds on $d_{K L}=\left|x_{K}-x_{L}\right|, d_{K^{*} L^{*}}=\left|x_{K^{*}}-x_{L^{*}}\right|$ :

\begin{tabular}{|l} 
For all primal neighbors $K, L, \quad \operatorname{diam}(K)+\operatorname{diam}(L) \leqslant \operatorname{reg}(\mathfrak{T}) d_{K L} ;$ \\
for all dual neighbors $K^{*}, L^{*}, \quad \operatorname{diam}\left(K^{*}\right)+\operatorname{diam}\left(L^{*}\right) \leqslant \operatorname{reg}(\mathfrak{T}) d_{K^{*} L^{*}} ;$ \\
for all diamond $D$ with vertices $x_{K}, x_{L}$ \\
and with neighbor dual vertices $x_{K^{*}}, x_{L^{*}}, \quad \operatorname{diam}(D) \leqslant \operatorname{reg}(\mathfrak{T}) \min \left\{d_{K L}, d_{K^{*} L^{*}}\right\}$.
\end{tabular}

Further, we need a bound on the inclination of the (primal and dual) interfaces with respect to the (dual or primal) edges (see Fig. 2):

For all primal neighbor volumes $K, L$, the angle $\alpha_{K, L}$ between $\overrightarrow{x_{K} x_{L}}$ and the plane $K \mid L$ is separated from 0 and $\pi$, meaning that $\operatorname{reg}(\boldsymbol{T}) \cos \alpha_{K, L} \geqslant 1 ;$ and

for all neighbor vertices $x_{K^{*}}, x_{L^{*}}$ of $K \mid L$, the angle $\alpha_{K^{*}, L^{*}}^{*}$ between $\overrightarrow{x_{K^{*}} x_{L^{*}}}$ and $\overrightarrow{x_{K^{*} \mid L^{*}} x_{K \mid L}}$ is separated from 0 and $\pi$, namely $\operatorname{reg}(\mathfrak{T}) \cos \alpha_{K^{*}, L^{*}}^{*} \geqslant 1$.

Also a uniform bound on the number of neighbors of volumes and diamonds is useful:

All primal volume $K$ has at $\operatorname{most} \operatorname{reg}(\boldsymbol{T})$ neighbor primal volumes;

all dual volume $K^{*}$ has at most $\operatorname{reg}(\mathfrak{T})$ neighbor dual volumes;

and all diamond $D$ has at $\operatorname{most} \operatorname{reg}(\mathfrak{T})$ vertices.

The last of the three assumptions in (11) is satisfied, e.g., if one works under assumptions $\left(M_{\Delta}\right)$ or $\left(M_{\square}\right)$.

Actually, for the results using Poincaré and Sobolev inequalities, assumptions $\left(M_{\Delta}\right)$ or $\left(M_{\square}\right)$ are imposed, in order to guarantee that the kernel of the discrete gradient operator is reduced to constant discrete functions. Moreover, for the Sobolev embedding inequalities and for strong compactness in $L^{q}, q>1$ we also require

For all primal volume $K$ and interface $K \mid L, \quad m_{K \mid L} d_{K L} \leqslant \operatorname{reg}(\boldsymbol{T}) \operatorname{Vol}(K)$;

For all dual volume $K$ and interface $K^{*} L^{*}, \quad m_{K^{*} \mid L^{*}} d_{K^{*} L^{*}} \leqslant \operatorname{reg}(\boldsymbol{T}) \operatorname{Vol}\left(K^{*}\right)$.

Whenever one is interested in error rate analysis, further constraints on the shape of volumes, dual volumes, diamonds, and their intersections are needed (see [12] for the 2D case and [26] for the 3D CeVEFE-DDFV case). In the 3D CeVe-DDFV case, shape-regularity constraints on the faces of the primal mesh would also be required. 


\subsection{Consistency of projections, discrete gradient and divergence operators}

Here we gather basic consistency results for the DDFV discretizations. Heuristically, for a given function $\varphi$ on $\Omega$, projection of $\varphi$ on a mesh $\boldsymbol{T}$ and subsequent application of the discrete gradient $\nabla^{\mathfrak{T}}$ should produce a discrete field sufficiently close (as $\operatorname{size}(\mathfrak{T})$ gets small) to $\nabla \varphi$. Similarly, for a given field $\overrightarrow{\mathcal{F}}$, the adequate projection on the mesh and the application of $\operatorname{div}^{\mathfrak{T}}$ to this projection should yield a discrete function close to div $\overrightarrow{\mathcal{F}}$. Actually, we state two different kinds of consistency results, those in a norm and those in a weaker formulation using duality.

We use two kinds of projection, the mean-value and the center-value ones. Namely, for scalar functions on $\Omega$, the following projections on $\mathbb{R}^{\mathfrak{I}}$ (which have two components, namely the projections on $\mathfrak{M}^{o}$ and on $\mathfrak{M}^{*}$ ) are used:

$$
\begin{gathered}
\mathbb{P}^{\mathfrak{T}}: \varphi \mapsto\left(\left(\frac{1}{\operatorname{Vol}(K)} \int_{K} \varphi\right)_{K \in \mathfrak{M}^{o}},\left(\frac{1}{\operatorname{Vol}\left(K^{*}\right)} \int_{K^{*}} \varphi\right)_{K^{*} \in \mathfrak{M}^{*}}\right)=:\left(\mathbb{P}^{\mathfrak{M}^{o}} \varphi, \mathbb{P}^{\mathfrak{M}^{*}} \varphi\right), \\
\mathbb{P}_{c}^{\mathfrak{T}}: \varphi \mapsto\left(\left(\varphi\left(x_{K}\right)\right)_{K \in \mathfrak{M}^{o}},\left(\varphi\left(x_{K^{*}}\right)\right)_{K^{*} \in \mathfrak{M}^{*}}\right)=:\left(\mathbb{P}_{c}^{\mathfrak{M}^{o}} \varphi, \mathbb{P}_{c}^{\mathfrak{M}^{*}} \varphi\right) .
\end{gathered}
$$

If, in addition, $\varphi$ is zero on $\partial \Omega$, then $\mathbb{P}_{c}^{\mathfrak{\tau}} \varphi \in \mathbb{R}^{\mathfrak{\tau}}$ is extended to $\mathbb{P}_{c}^{\overline{\boldsymbol{s}}} \varphi \in \mathbb{R}_{0}^{\overline{\boldsymbol{\tau}}} ; \mathbb{P}_{c}^{\overline{\boldsymbol{\Sigma}}} \varphi$ is the projection of $\varphi$ on $\mathbb{R}^{\overline{\mathfrak{s}} 5}$. For $\mathbb{R}^{3}$-valued fields on $\Omega$, we use the projection on $\left(\mathbb{R}^{3}\right)^{\mathcal{D}}$ by

$$
\overrightarrow{\mathbb{P}^{\mathfrak{I}}}: \overrightarrow{\mathcal{F}} \mapsto\left(\frac{1}{\operatorname{Vol}(D)} \int_{D} \overrightarrow{\mathcal{F}}\right)_{D \in \mathfrak{D}}
$$

Let us stress that for the study of weak compactness in Sobolev spaces and analysis of convergence of discrete solutions to a weak solution of PDEs, the consistency results can be formulated for source terms and for test functions only (and the consistency for $\operatorname{div}^{\mathfrak{I}} \circ \overrightarrow{\mathbb{P}}^{\mathfrak{T}}$ is formulated in a weak form, except on very symmetric meshes). These results are shown under the regularity restrictions $(9),(10),(11)$ on the mesh.

Proposition 3.1 (Consistency of discrete operators). Let $\mathfrak{T}$ be a $3 D$ CeVe-DDFV mesh of $\Omega$ satisfying the general assumption $(M)$. Assume that the number $\operatorname{reg}(\boldsymbol{T})$ quantify the mesh regularity in the sense $(9),(10),(11)$. Then

(i) For all $\varphi \in \mathcal{D}(\bar{\Omega})$ one has

$$
\left\|\varphi-\mathbb{P}^{\mathfrak{M}^{o}} \varphi\right\|_{L^{\infty}(\Omega)} \leqslant C(\varphi) \operatorname{size}(\mathfrak{T}), \quad\left\|\varphi-\mathbb{P}^{\mathfrak{M}^{*}} \varphi\right\|_{L^{\infty}(\Omega)} \leqslant C(\varphi) \operatorname{size}(\mathfrak{T})
$$

analogous properties hold for $\mathbb{P}_{c}^{\mathfrak{M}^{o}}, \mathbb{P}_{c}^{\mathfrak{M}^{*}}$. Similarly, for all $\overrightarrow{\mathcal{F}} \in(\mathcal{D}(\bar{\Omega}))^{3}$ one has

$$
\|\overrightarrow{\mathcal{F}}-\overrightarrow{\mathbb{P}} \mathfrak{\mathcal { F }} \overrightarrow{\mathcal{F}}\|_{L^{\infty}(\Omega)} \leqslant C(\overrightarrow{\mathcal{F}}) \operatorname{size}(\mathfrak{T}) .
$$

(ii) For all $\varphi \in \mathcal{D}(\Omega)$ one has

$$
\left\|\nabla \varphi-\nabla^{\mathfrak{T}}\left(\mathbb{P}_{c}^{\overline{\bar{s}} \varphi}\right)\right\|_{L^{\infty}(\Omega)} \leqslant C(\varphi, \operatorname{reg}(\mathfrak{T})) \operatorname{size}(\mathfrak{T}) .
$$

\footnotetext{
${ }^{5}$ In this paper, we only discuss DDFV discretizations of diffusion operators supplied with homogeneous Dirichlet or Neumann BC. In the homogeneous Dirichlet case, the boundary values of a discrete function are zero. In the homogeneous Neumann case, boundary values never appear. Consideration of non-homogeneous boundary conditions is a highly technical issue. We refer to the analysis of [12] for the Dirichlet case, and to $[21,62,7,23]$ for the non-homogeneous Neumann condition on a part of the boundary.
} 
(iii) Assume that the mesh is of the kind $\left(M_{\Delta}\right)$. Then for all $\overrightarrow{\mathcal{F}} \in(\mathcal{D}(\bar{\Omega}))^{3}$, for all $w^{\overline{\mathfrak{s}}} \in \mathbb{R}_{0}^{\bar{\Sigma}}$ one has

$$
\left|\left[\left[\mathbb{P}^{\mathfrak{T}}(\operatorname{div} \overrightarrow{\mathcal{F}})-\operatorname{div}^{\mathfrak{T}}\left(\overrightarrow{\mathbb{P}^{\mathfrak{T}}} \overrightarrow{\mathcal{F}}\right), w^{\mathfrak{T}}\right]\right]_{\Omega}\right| \leqslant C(\overrightarrow{\mathcal{F}}, \operatorname{reg}(\mathfrak{T})) \operatorname{size}(\mathfrak{T})\left\|\nabla^{\mathfrak{T}} w^{\overline{\mathfrak{T}}}\right\|_{L^{1}(\Omega)} .
$$

(iii-bis) $\quad$ Assume that the mesh is of the kind $\left(M_{\square}\right)$. Then for all $\overrightarrow{\mathcal{F}} \in(\mathcal{D}(\bar{\Omega}))^{3}$,

$$
\left\|\mathbb{P}^{\mathfrak{T}}(\operatorname{div} \overrightarrow{\mathcal{F}})-\operatorname{div}^{\mathfrak{T}}\left(\overrightarrow{\mathbb{P}^{\mathfrak{T}}} \overrightarrow{\mathcal{F}}\right)\right\|_{L^{\infty}(\Omega)} \leqslant C(\overrightarrow{\mathcal{F}}) \operatorname{size}(\mathfrak{T})
$$

Remark 1.

(i) One can perceive (iii),(iii-bis) as a kind of commutation relation, but actually these items also express consistency results. Indeed, by (i), $\mathbb{P}^{\mathfrak{T}}(\operatorname{div} \overrightarrow{\mathcal{F}})$ is $\operatorname{size}(\boldsymbol{T})$-close to $\operatorname{div} \overrightarrow{\mathcal{F}}$ in $L^{\infty}(\Omega)$. Therefore the item (iii) can be interpreted as a size $(\mathfrak{T})$-smallness of $\left(\operatorname{div} \overrightarrow{\mathcal{F}}-\operatorname{div}^{\mathfrak{T}}\left(\overrightarrow{\mathbb{P}^{\mathfrak{T}}} \overrightarrow{\mathcal{F}}\right)\right)$ in the weak-* topology of $W^{-1, \infty}(\Omega)=\left(W_{0}^{1,1}(\Omega)\right)^{*}$. Similarly, the item (iii-bis) means that, for the uniform Cartesian mesh, $\left(\operatorname{div} \overrightarrow{\mathcal{F}}-\operatorname{div}^{\mathfrak{T}}\left(\overrightarrow{\mathbb{P}^{\mathfrak{x}}} \overrightarrow{\mathcal{F}}\right)\right)$ is of order $\operatorname{size}(\mathfrak{T})$ in $L^{\infty}$ norm.

(ii) The items (iii),(iii-bis) reflect a technical difficulty specific to the DDFV context. Indeed, in the context of finite volume schemes with two-point gradient reconstruction (iii),(iii-bis) holds just with $C=0$, for an appropriately defined field projection operator. To be specific, instead of the DDFV double mesh $\mathfrak{T}$ consider the primal mesh $\mathfrak{M}^{\circ}$ alone. One keeps the same definition for diamonds $D^{K \mid L}$, but sets $\overrightarrow{\mathbb{P}}^{\mathfrak{M}^{o}} \overrightarrow{\mathcal{F}}=\left(\frac{1}{m_{K \mid L}} \int_{K \mid L} \overrightarrow{\mathcal{F}}\right)_{D^{K \mid L} \in \mathfrak{P}}$. One only looks at the first components of the DDFV operators $\mathbb{P}^{\mathfrak{T}}$ and $\operatorname{div}^{\mathfrak{T}}$ (it is natural to denote them $\mathbb{P}^{\mathfrak{M}^{\circ}}$ and $\operatorname{div}^{\mathfrak{M}^{\circ}}$, respectively). Then from the Green-Gauss formula it is straightforward that

$$
\forall \overrightarrow{\mathcal{F}} \in(\mathcal{D}(\bar{\Omega}))^{3} \quad \mathbb{P}^{\mathfrak{M}^{o}}\left(\operatorname{div}^{\mathfrak{M}}{ }^{o} \overrightarrow{\mathcal{F}}\right)=\operatorname{div}^{\mathfrak{M}^{o}}\left(\overrightarrow{\mathbb{P}}^{\mathfrak{M}^{o}} \overrightarrow{\mathcal{F}}\right)
$$

Because in the DDFV context, for $D=D_{K^{*} \mid L^{*}}^{K \mid L}$, the values $\frac{1}{m_{K \mid L}} \int_{K \mid L} \overrightarrow{\mathcal{F}}$ and $\frac{1}{m_{K^{*} \mid L^{*}}} \int_{K^{*} \mid L^{*}} \overrightarrow{\mathcal{F}}$ may differ, the analogue of the commutation relation (13) cannot be achieved, whatever definition is chosen for $\overrightarrow{\mathbb{P}^{\mathfrak{T}}}$.

Notice that error analysis for diffusion operators would require consistency properties also for functions $\varphi$ and fields $\overrightarrow{\mathcal{F}}$ in Sobolev spaces; this requires more regularity restrictions on the meshes, and much finer techniques (see e.g. [12]). Here, we only need the following additional property generalizing (i) and applicable to source terms:

Corollary 3.1. Let $p \in[1,+\infty)$. For all $\varphi \in L^{p}(\Omega)$ one has

$$
\left\|\varphi-\mathbb{P}^{\mathfrak{M}^{o}} \varphi\right\|_{L^{p}(\Omega)} \rightarrow 0, \quad\left\|\varphi-\mathbb{P}^{\mathfrak{M}^{*}} \varphi\right\|_{L^{p}(\Omega)} \rightarrow 0 \text { as size }(\boldsymbol{T}) \rightarrow 0 .
$$

ProOF : The proof is a straightforward combination of the density of $\mathcal{D}(\bar{\Omega})$ in $L^{p}(\Omega)$, of Proposition 3.1(i) and of the uniform boundedness of the projection operators $\mathbb{P}^{\mathfrak{M}^{\circ}}$, $\mathbb{P}^{\mathfrak{M}^{*}}$ seen as operators from $L^{p}(\Omega)$ to itself. 


\section{Proof of Proposition 3.1:}

(i) The properties are evident from the Lipschitz continuity of $\varphi$ and of $\overrightarrow{\mathcal{F}}$, respectively.

(ii) Here the consistency of the discrete gradient on affine functions, stated in Proposition 3.1 , is used together with the $C^{2}$ regularity of $\varphi$. Namely, we compare $\nabla^{\mathfrak{s}} \mathbb{P}^{\overline{\boldsymbol{s}}} \varphi$ to $\nabla \varphi$ diamond-wise. From the regularity assumption (9), it is clear that some $C(\operatorname{reg}(\mathfrak{T})) \operatorname{diam}(D)$ neighborhood $N_{D}$ of a given diamond $D=D^{K \mid L} \in \mathfrak{D}$ contains the two primal volumes $K, L$ and the dual volumes $K_{i}^{*}, i=1, \ldots, l$, used to reconstruct $\nabla_{D} \mathbb{P}^{\overline{\boldsymbol{\Sigma}}} \varphi$. Let $w$ denote the affine Taylor polynomial of $\varphi$ at some fixed point of $D$ (when $D$ touches the boundary $\partial \Omega$, we pick a point at the boundary and get $w \equiv 0)$. Then

$$
\|\nabla \varphi-\nabla w\|_{L^{\infty}(D)} \leqslant C(\varphi) \operatorname{size}(\boldsymbol{T}), \quad\left|\varphi_{K}-w_{K}\right|,\left|\varphi_{L}-w_{L}\right|,\left|\varphi_{K_{i}^{*}}-w_{K_{i}^{*}}\right| \leqslant C(\varphi)(\operatorname{diam}(D))^{2},
$$

where $\varphi_{K}, \varphi_{K_{i}^{*}}$, etc. denote the entries of the discrete function $\mathbb{P}_{c}^{\overline{\mathfrak{z}}} \varphi$, while $w_{K}:=w\left(x_{K}\right)$, $w_{K_{i}^{*}}:=w\left(x_{K_{i}^{*}}\right)$ are the values of $\mathbb{P}_{c}^{\overline{\mathbf{s}}} w$.

According to Proposition 3.1, the values $\nabla_{D} w^{\overline{\mathfrak{s}}}$ and $\left.\nabla w\right|_{D}$ coincide. In order to estimate the difference $\left\|\nabla \varphi-\nabla^{\mathfrak{\tau}}\left(\mathbb{P}^{\overline{\mathfrak{\Sigma}}} \varphi\right)\right\|_{L^{\infty}(D)}$, it remains to compare the values $\nabla_{D} w^{\overline{\mathfrak{\Sigma}}}$ and $\nabla_{D}\left(\mathbb{P}^{\overline{\mathfrak{\Sigma}}} \varphi\right)$. Notice that thanks to the previous $(\operatorname{diam}(D))^{2}$ bounds on $\left|\varphi_{K}-w_{K}\right|,\left|\varphi_{L}-w_{L}\right|$ and to the bound $\operatorname{reg}(\mathfrak{T}) d_{K L} \geqslant \operatorname{diam}(D)$ in $(9)$, we have

$$
\left|\frac{w_{L}-w_{K}}{d_{K L}}-\frac{\varphi_{L}-\varphi_{K}}{d_{K L}}\right| \leqslant C(\operatorname{reg}(\mathfrak{T})) \operatorname{diam}(D) \leqslant C(\operatorname{reg}(\mathfrak{T})) \operatorname{size}(\mathfrak{T}) .
$$

In view of the expression of the discrete gradient (formula (7)) and in view of the mesh inclination bound (10) (notice that $\cos \alpha_{K, L}$ appears as a factor in the denominator $\operatorname{Vol}(D)$ of formula (7)), the contribution of the values in $K, L$ into $\left|\nabla_{D} w^{\overline{\boldsymbol{s}}}-\nabla_{D}\left(\mathbb{P}^{\overline{\mathbf{x}}} \varphi\right)\right|$ is estimated by $C(\operatorname{reg}(\mathfrak{T})) \operatorname{size}(\boldsymbol{T})$. Looking closely at the $\operatorname{Proj}_{D}^{*}$ component in formula (8) (notice in particular that $\cos \alpha_{K_{i}^{*}, K_{i+1}^{*}}^{*}$ is a factor in the denominator $\operatorname{Vol}(D)$ in $\left.(8)\right)$, using in addition

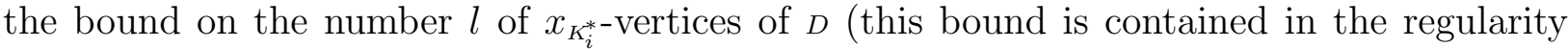
assumption (11)), we estimate in the same way the contribution of the values in $K_{i}^{*}$ into $\left|\nabla_{D} w^{\overline{\mathfrak{T}}}-\nabla_{D}\left(\mathbb{P}^{\overline{\mathfrak{x}}} \varphi\right)\right|$.

(iii) We refer to Section 2.2 for the notation used in the proof.

First, using the Green-Gauss formula, we can rewrite the value $(\operatorname{div} \overrightarrow{\mathcal{F}})_{K}$ of the discrete function $\mathbb{P}^{\mathfrak{r}}(\operatorname{div} \overrightarrow{\mathcal{F}})$ in $K$ under the form reminiscent of the form (5) of the discrete divergence operator:

$$
(\operatorname{div} \overrightarrow{\mathcal{F}})_{K}=\sum_{\mathrm{S} \sim K} \int_{\sigma_{\mathrm{S}}} \overrightarrow{\mathcal{F}} \cdot(-1)^{\epsilon_{S}^{K}} \vec{n}_{\mathrm{S}}=\sum_{\mathrm{S} \sim K} m_{\mathrm{S}} \overrightarrow{\mathcal{F}}_{\sigma_{\mathrm{S}}} \cdot(-1)^{\epsilon_{S}^{K}} \vec{n}_{\mathrm{S}}
$$

Here $\overrightarrow{\mathcal{F}}_{\sigma_{\mathrm{S}}}$ is the mean value of the field $\overrightarrow{\mathcal{F}}$ on the part $\sigma_{\mathrm{S}} \subset S$ of the primal interface defining the subdiamond $S$. One represents analogously the value $(\operatorname{div} \overrightarrow{\mathcal{F}})_{K^{*}}$ of $\mathbb{P}^{\mathfrak{r}}(\operatorname{div} \overrightarrow{\mathcal{F}})$ in $K^{*}$, using the mean values $\overrightarrow{\mathcal{F}}_{\sigma_{S_{\odot}}^{*}}, \overrightarrow{\mathcal{F}}_{\sigma_{\mathrm{S}_{\oplus}}^{*}}$ of $\overrightarrow{\mathcal{F}}$ on the parts $\sigma_{S_{\odot}}^{*}, \sigma_{S_{\oplus}}^{*} \subset S$ of the dual interface defining $S$. Recall that $S$ is contained in a diamond $D$ (or associated with $D$, see [3, Sec. 4]). It is convenient to define $\overrightarrow{\mathcal{F}}_{\mathrm{S}}:=\overrightarrow{\mathcal{F}}_{D}$ for all subdiamond $S$ of $D$. In turn, $\overrightarrow{\mathcal{F}}_{D}$ denotes the value in $D$ of the discrete field $\overrightarrow{\mathbb{P}^{\mathfrak{T}}} \overrightarrow{\mathcal{F}}$; recall that this is the mean value of the field $\overrightarrow{\mathcal{F}}$ on the diamond $D$. 
With this notation in hand, using the definition (3) of the scalar product $\left[[\cdot, \cdot]_{\Omega}\right.$ and the summation-by parts procedure (recall that $w^{\overline{\mathfrak{\tau}}}$ is zero on the boundary volumes), analogously to the proof of [3, Prop. 3.1] we get

$$
\begin{aligned}
& \left.\left.\llbracket \mathbb{P}^{\mathfrak{T}}(\operatorname{div} \overrightarrow{\mathcal{F}})-\operatorname{div}^{\mathfrak{T}}\left(\vec{P}^{\mathfrak{T}} \overrightarrow{\mathcal{F}}\right), w^{\mathfrak{T}}\right]\right]_{\Omega} \\
& =\frac{1}{3} \sum_{S \in \mathfrak{S}}\left(\left(m_{\mathrm{S}} d_{\odot, \oplus}\right) \frac{w_{\oplus}-w_{\odot}}{d_{\odot, \oplus}} \vec{n}_{\odot, \oplus} \cdot\left(\overrightarrow{\mathcal{F}}_{\sigma_{\mathrm{S}}}-\overrightarrow{\mathcal{F}}_{\mathrm{S}}\right)\right. \\
& \left.\quad+2 \frac{\left(w_{i+1}^{*}-w_{i}^{*}\right)}{d_{i, i+1}^{*}}\left\{\left(m_{\mathrm{S}_{\odot}}^{*} d_{i, i+1}^{*}\right) \vec{n}_{\mathrm{S}_{\odot}}^{*} \cdot\left(\overrightarrow{\mathcal{F}}_{\sigma_{\odot}^{*}}-\overrightarrow{\mathcal{F}}_{\mathrm{S}}\right)+\left(m_{\mathrm{S}_{\oplus}}^{*} d_{i, i+1}^{*}\right) \vec{n}_{\mathrm{S}_{\oplus}}^{*} \cdot\left(\overrightarrow{\mathcal{F}}_{\sigma_{\mathrm{S}_{\oplus}^{*}}^{*}}-\overrightarrow{\mathcal{F}}_{\mathrm{S}}\right)\right\}\right) .
\end{aligned}
$$

Here $m_{\mathrm{S}}, m_{\mathrm{S}_{\odot}}^{*}, m_{\mathrm{S} \oplus}^{*}$ are two-dimensional measures of parts of faces represented in Fig. 2, and the summation runs over all subdiamonds $s \in \mathfrak{S}$ represented as $S=S_{K_{i}^{*} \mid K_{*+1}^{*}}^{K_{*}^{*}}$. Now, notice that the mesh inclination bound (10) implies that for a subdiamond $S=S_{K_{i}^{*} \mid K_{i+1}^{*}}^{K_{\oplus} \mid}$,

$$
\operatorname{reg}(\boldsymbol{T}) \operatorname{Vol}\left(S_{K_{i}^{*}\left|K_{i+1}^{*}\right| K_{\oplus}}^{K_{1+1}}\right) \geqslant m_{\mathrm{S}} d_{\odot, \oplus}, \quad \operatorname{reg}(\mathfrak{T}) \operatorname{Vol}\left(S_{K_{i}^{*} \mid K_{i+1}^{*}}^{K_{\oplus} \mid K_{\oplus}}\right) \geqslant m_{\mathrm{S}_{\odot}}^{*} d_{i, i+1}^{*}+m_{\mathrm{S}_{\oplus}}^{*} d_{i, i+1}^{*},
$$

for the case of the meshes described in [3, Sec. 2]. Remark that, if one allows for subdiamonds of negative volume as in [3, Sec. 4], then (14) may loose sense; yet exploiting the restriction on the number of vertices of $k \mid L$, one can replace the bound (14) used in the below calculation by the bound

$$
\operatorname{reg}(\mathfrak{T}) \operatorname{Vol}\left(D^{K_{\odot} \mid K_{\oplus}}\right) \geqslant m_{\mathrm{S}} d_{\odot, \oplus}, \quad \operatorname{reg}(\mathfrak{T}) \operatorname{Vol}\left(D^{K_{\odot} \mid K_{\oplus}}\right) \geqslant m_{\mathrm{S} \odot}^{*} d_{i, i+1}^{*}+m_{\mathrm{S} \oplus}^{*} d_{i, i+1}^{*},
$$

which is always true.

From the Lipschitz continuity of $\overrightarrow{\mathcal{F}}$, it is clear that

$$
\left|\overrightarrow{\mathcal{F}}_{\sigma_{\mathrm{S}}}-\overrightarrow{\mathcal{F}}_{\mathrm{S}}\right|,\left|\overrightarrow{\mathcal{F}}_{\sigma_{S_{\odot}}^{*}}-\overrightarrow{\mathcal{F}}_{\mathrm{S}}\right|,\left|\overrightarrow{\mathcal{F}}_{\sigma_{\mathrm{S}_{\oplus}}^{*}}-\overrightarrow{\mathcal{F}}_{\mathrm{S}}\right| \leqslant C(\overrightarrow{\mathcal{F}}) \operatorname{size}(\mathfrak{T}) ;
$$

hence using (14), we get the estimate

$$
\begin{aligned}
& \left.\mid \| \mathbb{P}^{\mathfrak{T}}(\operatorname{div} \overrightarrow{\mathcal{F}})-\operatorname{div}^{\mathfrak{T}}\left(\overrightarrow{\mathbb{P}}^{\mathfrak{T}} \overrightarrow{\mathcal{F}}\right), w^{\mathfrak{T}}\right]_{\Omega} \mid \\
& \qquad C(\overrightarrow{\mathcal{F}}, \operatorname{reg}(\mathfrak{T})) \operatorname{size}(\mathfrak{T}) \sum_{S \in \mathfrak{S}} \operatorname{Vol}(S)\left(\frac{\left|w_{\oplus}-w_{\odot}\right|}{d_{\odot, \oplus}}+\frac{\left|w_{i+1}^{*}-w_{i}^{*}\right|}{d_{i, i+1}^{*}}\right) .
\end{aligned}
$$

It remains to notice that if $\nabla_{S} w^{\bar{\Sigma}}=\nabla_{D} w^{\bar{\tau}}$ denotes the value in $S \sim D$ (which means $S \subset D$, for the meshes of $\left[3\right.$, Sec. 2]) of the discrete gradient $\nabla^{\mathfrak{T}} w^{\overline{\mathfrak{z}}}$, then

$$
\frac{\left|w_{\oplus}-w_{\odot}\right|}{d_{\odot, \oplus}}=\left|\operatorname{Proj}_{D}\left(\nabla_{D} w^{\overline{\tilde{\tau}}}\right)\right| \leqslant\left|\nabla_{D} w^{\overline{\mathfrak{\tau}}}\right| .
$$

In addition, because the dual interface $K_{\odot} \mid K_{\oplus}$ is assumed to be a triangle (assumption $\left(M_{\Delta}\right)$ ), according to [3, page 6 and Rem. A.2] each divided difference $\frac{w_{i+1}^{*}-w_{i}^{*}}{d_{i, i+1}^{*}}$ is precisely the projection 
of the $2 D$ vector $\operatorname{Proj}_{D}^{*}\left(\nabla_{D} w^{\bar{\tau}}\right)$ on the direction of $\overrightarrow{x_{i+1}^{*} x_{i}^{*}}$. Therefore we also have ${ }^{6}$

$$
\frac{\left|w_{i+1}^{*}-w_{i}^{*}\right|}{d_{i, i+1}^{*}} \leqslant\left|\operatorname{Proj}_{D}^{*}\left(\nabla_{D} w^{\bar{\tau}}\right)\right| \leqslant\left|\nabla_{D} w^{\bar{\tau}}\right| .
$$

Combining (15) with the two latter estimates, we deduce

$$
\begin{aligned}
& \left.||\left[\mathbb{P}^{\mathfrak{T}}(\operatorname{div} \overrightarrow{\mathcal{F}})-\operatorname{div}^{\mathfrak{T}}\left(\overrightarrow{\mathbb{P}}^{\mathfrak{\mathfrak { T }}} \overrightarrow{\mathcal{F}}\right), w^{\mathfrak{T}}\right]\right]_{\Omega}\left|\leqslant C(\overrightarrow{\mathcal{F}}, \operatorname{reg}(\mathfrak{T})) \operatorname{size}(\mathfrak{T}) \sum_{S \in \mathfrak{S}} \operatorname{Vol}(S)\right| \nabla_{\mathrm{S}} w^{\overline{\mathfrak{T}}} \mid \\
& \quad=C(\overrightarrow{\mathcal{F}}, \operatorname{reg}(\mathfrak{T})) \operatorname{size}(\mathfrak{T}) \sum_{D \in \mathfrak{D}} \operatorname{Vol}(D)\left|\nabla_{D} w^{\overline{\mathfrak{T}}}\right|=C(\overrightarrow{\mathcal{F}}, \operatorname{reg}(\mathfrak{T})) \operatorname{size}(\mathfrak{T})\left\|\nabla^{\mathfrak{T}} w^{\overline{\mathfrak{T}}}\right\|_{L^{1}(\Omega)} .
\end{aligned}
$$

(iii-bis) Although (16) is not fulfilled on Cartesian mesh, we gain even better consistency property due to the symmetry of the mesh. Indeed, let $\overrightarrow{\mathcal{F}}_{D}, \overrightarrow{\mathcal{F}}_{K L L}$ and $\overrightarrow{\mathcal{F}}_{K^{*} L^{*}}$ denote the mean values of $\overrightarrow{\mathcal{F}}$ on $D=D_{K^{*} L^{*}}^{K \mid}$, on $K \mid L$ and on $K^{*} L^{*}$, respectively. The claim stems from the fact that on a uniform Cartesian mesh, the differences $\overrightarrow{\mathcal{F}}_{D}-\overrightarrow{\mathcal{F}}_{K \mid L}, \overrightarrow{\mathcal{F}}_{D}-\overrightarrow{\mathcal{F}}_{K^{*} \mid L^{*}}$ are upper bounded by $C(\overrightarrow{\mathcal{F}})(\operatorname{size}(\mathfrak{T}))^{2}$. This follows from the cancellation of the order one terms in the Taylor expansion of $\overrightarrow{\mathcal{F}}$ (e.g., we expand $\overrightarrow{\mathcal{F}}$ at each point of $K \mid L$ and use the expansion to calculate the mean value $\overrightarrow{\mathcal{F}}_{D}$ on the diamond $D=D_{K^{*} L^{*}}^{K \mid L}$ which is symmetrical with respect to $\left.K \mid L\right)$.

Further, let $K$ be an interior primal volume. For the six diamonds that intersect $K$, we introduce the specific notation $D_{\text {abv }}(K), D_{\text {blw }}(K), D_{\mathrm{E}}(K), D_{\mathrm{W}}(K)$ and $D_{\mathrm{N}}(K), D_{\mathrm{S}}(K)$ with the obvious meaning of the subscripts (e.g., the diamond pictured on the right in Figure 1 is $D_{\text {blw }}\left(K_{\oplus}\right)$ and at the same time, it is $\left.D_{\text {abv }}\left(K_{\odot}\right)\right)$. Further, denote by $\sigma_{\mathrm{E}}(K), \sigma_{\mathrm{W}}(K), \sigma_{\mathrm{N}}(K), \sigma_{\mathrm{S}}(K)$, $\sigma_{\mathrm{abv}}(K), \sigma_{\mathrm{blw}}(K)$ the six interfaces surrounding $K$. Applying the evident formula for discrete divergence (see [3, Sect. 2.3, page 13]), using the the Green-Gauss theorem, as in the proof of (iii) we can write

$\mathbb{P}^{\mathfrak{T}}(\operatorname{div} \overrightarrow{\mathcal{F}})-\operatorname{div}^{\mathfrak{T}}\left(\overrightarrow{\mathbb{P}}^{\mathfrak{T}} \overrightarrow{\mathcal{F}}\right)=\frac{1}{1 / N^{3}}\left\{\frac{1}{N^{2}}\left(\overrightarrow{\mathcal{F}}_{\sigma_{\mathrm{E}}(K)}-\overrightarrow{\mathcal{F}}_{D_{\mathrm{E}}(K)}\right) \cdot \vec{i}+\cdots+\frac{1}{N^{2}}\left(\overrightarrow{\mathcal{F}}_{\sigma_{\mathrm{blw}}(K)}-\overrightarrow{\mathcal{F}}_{D_{\mathrm{blw}}(K)}\right) \cdot(-\vec{k})\right\}$.

Thus the claim of (iii-bis) is direct from the above bound of order $(\operatorname{size}(\boldsymbol{T}))^{2}=\frac{1}{N^{2}}$ on the differences $\overrightarrow{\mathcal{F}}_{D_{\mathrm{E}}(K)}-\overrightarrow{\mathcal{F}}_{\sigma_{\mathrm{E}}(K)}, \ldots, \overrightarrow{\mathcal{F}}_{D_{\mathrm{blw}}(K)}-\overrightarrow{\mathcal{F}}_{\sigma_{\mathrm{blw}}(K)}$.

\subsection{Discrete Poincaré inequality, Sobolev embeddings and strong compactness}

Discrete embedding inequalities have been treated extensively in the finite volume literature. We refer to [33, 41, 13, 42, 52, 66, 22] for various approaches and results on different kinds of $2 \mathrm{D}$ and $3 \mathrm{D}$ schemes. For instance, paper [22] contains a section devoted specifically to Sobolev inequalities for 2D DDFV schemes. Yet the proofs of Poincaré and Sobolev inequalities need not be specific to the DDFV case (except if one is interested in the optimal value of the embedding constant, as it is the case in the work [66]). Indeed, the key fact for the 3D CeVe-DDFV scheme is the following remark:

Assuming $\left(M_{\Delta}\right)$ (e.g, each face $K \mid L$ of the mesh $\mathfrak{M}^{o}$ is a triangle), one gets the same embedding results on the 3D CeVe-DDFV meshes as the results known for the two-point discrete gradients on $\mathfrak{M}^{o}$ and on $\mathfrak{M}^{*}$.

\footnotetext{
${ }^{6}$ only at this point the assumption of triangular faces is needed
} 
Indeed, it has been already observed in the proof of Proposition 3.1(iii) that the restriction $l=3$ on the number $l$ of dual vertices of a diamond $D^{K_{\odot} \mid K_{\oplus}}$ allows for a control by $\left|\nabla_{D} w^{\overline{\bar{x}}}\right|$ of the divided differences:

$$
\frac{\left|w_{\oplus}-w_{\odot}\right|}{d_{\odot, \oplus}} \leqslant\left|\nabla_{D} w^{\overline{\underline{\tau}}}\right|, \quad \frac{\left|w_{i+1}^{*}-w_{i}^{*}\right|}{d_{i, i+1}^{*}} \leqslant\left|\nabla_{D} w^{\overline{\mathrm{s}}}\right|
$$

(here $i=1,2,3$ and by our convention, $w_{4}^{*}:=w_{1}^{*}, d_{3,4}:=d_{1,3}$; see Fig. 2). Therefore for a proof of the different embeddings, we can treat the primal and the dual meshes in $\mathfrak{T}$ separately, as if our scheme was a scheme with the two-point gradient reconstruction.

Note that, when the number $l$ of vertices of a face $K \mid L$ exceeds three, the kernel of the linear form (7) used to reconstruct the discrete gradient in $D^{K \mid L}$ is not reduced to discrete functions constant at the vertices of $K \mid L$. If the number $l$ of vertices of a face of $\mathfrak{M}^{o}$ is large, it is not difficult to construct examples of non-zero discrete functions on $\Omega$, null on $\partial \Omega$, and with non-zero discrete gradient. In general, the situation with $l \leqslant 4$ vertices is not clear; e.g. the discrete Poincaré inequality holds on every individual mesh, but it is not an easy task to prove that the embedding constant is uniform, even under rigid proportionality assumptions on the meshes. The uniform Cartesian meshes is one case with $l=4$ that can be treated thoroughly, see Proposition 3.3 for the Poincaré inequality. Yet Remark 4 below shows that a control on the discrete gradient still allows for oscillations in discrete solutions, thus compactness of embeddings may fail. Indeed, the compactness of sub-critical embeddings is false when the primal meshes with quadrangular faces are considered, unless the solution on the dual mesh $\mathfrak{M}^{*}$ is further separated into two components (actually, this corresponds to a 3D CeVeFE-DDFV scheme in the spirit of [26]). Finally, from the numerical point of view, higher values of $l$ do not lead to troubles for the test cases we have examined (see [14] and [3, Sec. 5]).

Let us first give discrete DDFV versions of the embeddings of the discrete $W_{0}^{1, p}(\Omega)$ spaces. We mean the embedding into $L^{p}(\Omega)$ (the Poincaré inequality), into $L^{p^{*}}(\Omega)$ with $p^{*}:=\frac{3 p}{3-p}$, $p<3$ (the critical Sobolev embedding), as well as the compact embeddings into $L^{q}(\Omega)$ for all $q<p^{*}$.

\section{Proposition 3.2 (Poincaré-Sobolev inequalities, case of triangular faces).}

(i) Let $\mathfrak{T}$ be a $3 D$ CeVe-DDFV mesh of $\Omega$ satisfying $\left(M_{\Delta}\right)$. Let $\operatorname{reg}(\mathfrak{T})$ quantify the mesh regularity in the sense (10) and (12).

Let $w^{\overline{\mathfrak{s}}} \in \mathbb{R}_{0}^{\overline{\mathfrak{\tau}}}$. Then for all $p \in[1,+\infty)$,

$$
\left\|w^{\mathfrak{M}^{o}}\right\|_{L^{p}(\Omega)},\left\|w^{\mathfrak{M}^{*}}\right\|_{L^{p}(\Omega)} \leqslant C(p, \Omega, \operatorname{reg}(\mathfrak{T}))\left\|\nabla^{\mathfrak{T}} w^{\overline{\mathfrak{s}}}\right\|_{L^{p}(\Omega)} .
$$

Moreover, if $p \in[1,3)$ and $p^{*}:=\frac{3 p}{3-p}$, then

$$
\left\|w^{\mathfrak{M}^{o}}\right\|_{L^{p^{*}}(\Omega)},\left\|w^{\mathfrak{M}^{*}}\right\|_{L^{p^{*}}(\Omega)} \leqslant C(p, \Omega, \operatorname{reg}(\mathfrak{T}))\left\|\nabla^{\mathfrak{T}} w^{\overline{\mathfrak{x}}}\right\|_{L^{p}(\Omega)} .
$$

(ii) Let $w^{\overline{\boldsymbol{s}}_{h}} \in \mathbb{R}_{0}^{\overline{\boldsymbol{\tau}}_{h}}$ be discrete functions on a family $\left(\boldsymbol{\mathfrak { T }}_{h}\right)_{h}$ of $3 D$ CeVe-DDFV meshes of $\Omega$ satisfying $\left(M_{\Delta}\right)$, parametrized by $h \geqslant \operatorname{size}\left(\boldsymbol{T}_{h}\right)$. Assume that $\sup _{h \in\left(0, h_{\text {max }}\right]} \operatorname{reg}\left(\boldsymbol{T}_{h}\right)<+\infty$, where $\operatorname{reg}\left(\boldsymbol{\mathfrak { T }}_{h}\right)$ quantifies the regularity of $\boldsymbol{\mathfrak { T }}_{h}$ in the sense (10) and (12).

Assume that the family $\left(\nabla^{\mathfrak{x}_{h}} w^{\overline{\mathfrak{x}}_{h}}\right)_{h \in\left(0, h_{\max }\right]}$ is bounded in $L^{p}(\Omega)$ for some $p<+\infty$.

Then for all sequence $\left(h_{i}\right)_{i}$ converging to zero, each of the families $\left(w^{\mathfrak{M}^{o}} h_{i}\right)_{i},\left(w^{\mathfrak{M}_{h_{i}}^{*}}\right)_{i}$ is relatively compact in $L^{q}(\Omega)$ for all $q<+\infty$ (if $p \geqslant 3$ ) or $q<p^{*}($ if $1 \leqslant p<3$ ). 
Notice that for the Poincaré inequality (the first statement of (i)), the assumption (12) is not needed: we refer to [13] for a proof of this fact. Actually, with the hint of [13, Lemma 2.6] the Sobolev embeddings for $q \leqslant p \times 1^{*}=\frac{3 p}{2}$ can be obtained without using (12).

The statements (i),(ii) follow in a very direct way from the proofs given in [41, 24, 42]. Because of (17), the assumption that the primal mesh faces are triangles (i.e., $l=3$ ) is a key assumption for the proof. In some of the proofs we refer to, admissibility assumptions on the mesh (such as the mesh orthogonality and assumptions of the kind " $\left|x_{K}-x_{L}\right| \leqslant$ $\operatorname{reg}(\mathfrak{T})\left|x_{K}-x_{K \mid L}\right| "$, see $[41,42]$ ) were imposed. Yet, as in [12] (where the proof of the Poincaré inequality is given for the $2 D$ case), these assumptions are easily replaced with the bounds

$$
\begin{aligned}
m_{K \mid L} d_{K L} & \leqslant C(\operatorname{reg}(\mathfrak{T})) \min \left\{\operatorname{Vol}\left(D^{K \mid L}\right), \operatorname{Vol}(K), \operatorname{Vol}(L)\right\}, \\
m_{K^{*} \mid L^{*}} d_{K^{*} L^{*}} & \leqslant C(\operatorname{reg}(\mathfrak{T})) \min \left\{\operatorname{Vol}\left(S_{K^{*} \mid L^{*}}^{K \mid L}\right), \operatorname{Vol}\left(K^{*}\right), \operatorname{Vol}\left(L^{*}\right)\right\}
\end{aligned}
$$

that stem from the mesh regularity assumptions (12) and (10).

Remark 2. The corresponding embeddings of the discrete space $W^{1, p}(\Omega)$ contain an additional term in the right-hand side, which is usually taken to be either the mean value of $w^{\bar{s}}$ on some fixed part $\Gamma$ of the boundary $\partial \Omega$ (used when a non-homogeneous Dirichlet boundary condition on $\Gamma$ is imposed), or the mean value of $w^{\bar{\Sigma}}$ on some subdomain $\omega$ of $\Omega$ (the simplest choice is $\omega=\Omega$, used for the pure Neumann boundary conditions). Let us point out that the strategy of Eymard, Gallouët and Herbin in [42] actually allows to obtain Sobolev embeddings for the "Neumann case" as soon as the Poincaré inequality is obtained. For the proof, one bootstraps the estimate of $\int_{\Omega}|u|^{\alpha}$. First obtained from the Poincare inequality with $\alpha=p$, it is extended to $\alpha=p \times 1^{*}$ with the discrete variant [42, Lemma 5.2] (where one can exploit (18)) of the Nirenberg technique. In the same way, the bound of $\int_{\Omega}|u|^{\alpha}$ is further extended to $\alpha=p\left(1^{*}\right)^{2}$ and so on, until one reaches the critical exponent $p^{*}$. The details are given in [8]. Moreover, the Poincaré inequality for the "Neumann case" and $p=2$ (i.e., the embedding into $L^{2}(\Omega)$ of the discrete space $\left.\left\{u \in H^{1}(\Omega) \mid \int_{\Omega} u=0\right\}\right)$ was shown in [42], [52]. The assumption that $p=2$ is not essential in these proofs, and thus we can consider that the analogue of Proposition 3.2 with the additional terms $\left|\frac{1}{\operatorname{Vol}(\Omega)} \int_{\Omega} w^{\mathfrak{M}^{o}}\right|,\left|\frac{1}{\operatorname{Vol}(\Omega)} \int_{\Omega} w^{\mathfrak{M}^{*}}\right|$ in the right-hand side of the estimates is justified.

Remark 3. Notice that the same arguments that yield the Poincaré inequality with zero boundary condition yield the trace inequalities of the kind

$$
\left\|w^{\overline{\mathfrak{M}^{o}}}\right\|_{L^{p}(\Gamma)} \leqslant C(\Gamma, \Omega, \operatorname{reg}(\mathfrak{T}), p)\left(\left\|w^{\mathfrak{M}^{o}}\right\|_{L^{p}(\Omega)}+\left\|\nabla^{\mathfrak{T}} w^{\overline{\mathfrak{T}}}\right\|_{L^{p}(\Omega)}\right)
$$

(the inequality on the dual mesh is completely analogous). These inequalities are useful for treating non-homogeneous Neumann boundary conditions on a part $\Gamma$ of $\partial \Omega$ (see e.g. [7]).

Now we treat the case of uniform Cartesian DDFV meshes.

Proposition 3.3 (Poincaré-Sobolev inequalities, case of Cartesian meshes). Let $\Omega$ be the unit cubic domain, $N \in \mathbb{N}$ and let $\boldsymbol{T}$ be the mesh of $\Omega$ satisfying $\left(M_{\square}\right)$. Then the two claims of Proposition 3.2(i) (as well as the corresponding "Neumann-case" embedding inequalities with the additional term $\left|\frac{1}{\operatorname{Vol}(\Omega)} \int_{\Omega} w^{\mathfrak{T}}\right|$ in the right-hand side) still hold true.

Proof : Notice that we cannot apply directly the previous arguments because (17) is false for the cubic meshes. Indeed, for a cubic mesh described in Section 2.1, the discrete 
gradient in a diamond $D=D^{K_{\odot} \mid K_{\oplus}}$ is given by

$$
\nabla_{D} w^{\mathfrak{z}}=\frac{w_{K_{3}^{*}}-w_{K_{1}^{*}}}{\sqrt{2} / N}(\vec{i}+\vec{j})+\frac{w_{K_{4}^{*}}-w_{K_{2}^{*}}}{\sqrt{2} / N}(\vec{j}-\vec{i})+\frac{w_{K_{\oplus}}-w_{K_{\odot}}}{1 / N} \vec{k}
$$

(see Fig. 1 for the notation) where $1 / N$ is the edge length. Clearly, $\nabla_{D} w^{\mathfrak{z}}$ controls the divided differences $\left|\frac{w_{K_{\oplus}}-w_{K_{\odot}}}{1 / N}\right|$ of the values of $w^{\mathfrak{T}}$ in all neighbor primal volumes $K_{\odot}, K_{\oplus}$, and also the divided differences $\left|\frac{w_{K_{i+2}^{*}}-w_{K_{i}^{*}}}{\sqrt{2} / N}\right|$ along the diagonals of the faces of primal volumes.

Therefore the arguments that justify Proposition 3.2(i) also yield the embedding estimates for $w^{\mathfrak{m}^{o}}$. Further, let us replace the dual mesh $\overline{\mathfrak{M}^{*}}$ by two meshes $\overline{\mathfrak{M}_{\mathfrak{e}}^{*}}, \overline{\mathfrak{M}_{\mathfrak{o}}^{*}}$ such that the edges of each mesh are either the diagonals of the faces of primal volumes, or parts of $\partial \Omega$. Then the same arguments as in Proposition 3.2(i) apply to each of the two new meshes. To be specific, the family $\overline{\mathfrak{M}^{*}}$ of the dual volumes (their centers $\left(x_{K^{*}}\right)_{K^{*} \in \overline{\mathfrak{M}^{*}}}$ form a uniform Cartesian net of $\Omega=[0,1]^{3}$ ) is split into two families $\mathfrak{e}^{*}, \mathfrak{o}^{*}$. Namely, if a vertex $x_{K^{*}}$ has the coordinates $\left(\frac{n_{x}}{N}, \frac{n_{y}}{N}, \frac{n_{z}}{N}\right)$ in the canonic coordinates of $\mathbb{R}^{3}$, then the corresponding volume $K^{*}$ belongs to the family $\mathfrak{e}^{*}$ whenever $n_{x}+n_{y}+n_{z}$ is even, and it belongs to the family $\mathfrak{o}^{*}$ otherwise. Inside $\Omega$, one can connect the dual vertices $\left(x_{K^{*}}\right)_{K^{*} \in \mathfrak{e}^{*}}$ into a uniform tetrahedral graph of edge length $\sqrt{2} / N$; the same is true for $\left(x_{K^{*}}\right)_{K^{*} \in \mathfrak{o}^{*}}$. Now, the meshes $\overline{\mathfrak{M}_{\mathfrak{e}}^{*}}$ and $\overline{\mathfrak{M}_{\mathfrak{o}}^{*}}$

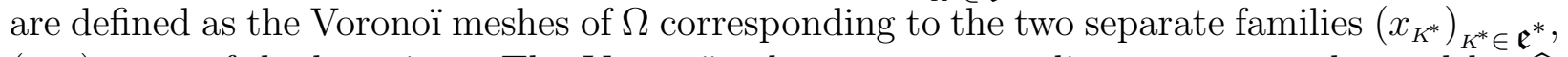
$\left(x_{K^{*}}\right)_{K^{*} \in \mathfrak{o}^{*}}$ of dual vertices. The Voronoï volumes corresponding to $x_{K^{*}}$ are denoted by $\widehat{K^{*}}$ (thus the union for $K^{*} \in \mathfrak{e}^{*} \cup \mathfrak{o}^{*}$ of all Voronoï volumes $\widehat{K^{*}}$ covers $\Omega$ twice). If we define

$$
w^{\mathfrak{M} \mathfrak{c}^{*}}:=\sum_{K^{*} \in \mathfrak{e}^{*}} w_{K^{*}} 1_{\widehat{K^{*}}}, \quad w^{\mathfrak{M} *}:=\sum_{K^{*} \in \mathfrak{o}^{*}} w_{K^{*}} 1_{\widehat{K^{*}}},
$$

then the embedding estimates of Proposition 3.2(i) are valid for $w^{\mathfrak{M}_{\varepsilon}^{*}}$ and for $w^{\mathfrak{M}_{\mathrm{r}}^{*}}$. By construction, $w^{\mathfrak{M}^{*}}$ takes alternatively the values of $w^{\mathfrak{M}_{\mathbf{*}}^{*}}$ and $w^{\mathfrak{M}_{\mathbf{6}}^{*}}$; more precisely,

$$
w^{\mathfrak{M}^{*}}=w^{\mathfrak{M}_{\mathfrak{k}}^{*}}\left(\sum_{K^{*} \in \mathfrak{e}^{*}} 1_{K^{*}}\right)+w^{\mathfrak{M}_{\mathfrak{o}}^{*}}\left(\sum_{K^{*} \in \mathfrak{o}^{*}} 1_{K^{*}}\right) .
$$

Thus $\left|w^{\mathfrak{M}^{*}}\right| \leqslant \max \left\{w^{\mathfrak{M}_{\mathfrak{*}}^{*}}, w^{\mathfrak{M}_{\mathfrak{b}}^{*}}\right\}$, and we get the desired estimates for $\left|w^{\mathfrak{M}^{*}}\right|$.

Remark 4. In the situation of Proposition 3.3, the compactness claim from Proposition 3.2(ii) gets wrong. More precisely, the compactness of the families $\left(w^{\mathfrak{M}_{h}^{*}}\right)_{h},\left(w^{\mathfrak{M}_{h}^{*} h}\right)_{h}$, and $\left(w^{\mathfrak{M}_{h}^{*}}\right)_{h}$ is true. This is shown with the same arguments borrowed from $[24,41,42]$. Yet let us stress that in general, there is no relation between the accumulation points of the three families. Therefore one can see from formula $(20)$ that the family $\left(w^{\mathfrak{M}^{*}} h\right)_{h}$ may present oscillations.

Let us illustrate the remark with an example. Take a smooth function $w \in \mathcal{D}(\Omega)$, non identically zero, and set

$$
\forall K \in \mathfrak{M}^{o} \quad w_{K}:=0 ; \quad \forall x_{K^{*}} \in \mathfrak{e}^{*} \quad w_{K^{*}}:=0 ; \quad \forall x_{K^{*}} \in \mathfrak{o}^{*} \quad w_{K^{*}}:=w\left(x_{K^{*}}\right) .
$$

The discrete gradient $\nabla^{\mathfrak{T}}$ does not couple the two families $\mathfrak{e}^{*}, \mathfrak{o}^{*}$ of dual vertices (see the proof of Proposition 3.3), therefore the discrete gradient $\nabla^{\mathfrak{T}} w^{\overline{\mathfrak{s}}}$ is bounded pointwise 
by $\|\nabla w\|_{L^{\infty}(\Omega)}$. Yet the family $w^{\mathfrak{M}^{*}}$ oscillates. Indeed, while $w^{\mathfrak{M}_{\varepsilon}^{*}}$ is identically zero, $w^{\mathfrak{M}_{0}^{*}}$ converges in $L^{\infty}(\Omega)$, as $\operatorname{size}(\boldsymbol{T})$ goes to 0 , to the non-zero function $w$. It is easy to see from (20) that $w^{\mathfrak{M}^{*}}$ converges weakly in $L^{q}(\Omega), q<+\infty$, to the same limit as $\frac{1}{2}\left(w^{\mathfrak{M}_{\mathfrak{k}}^{*}}+w^{\mathfrak{M}_{\mathfrak{o}}^{*}}\right)$; and the latter function converges strongly to $\frac{1}{2}(0+w)=\frac{w}{2}$. The family of the differences $\left(w^{\mathfrak{M}^{*}}-\frac{w}{2}\right)$ weakly converges to zero; yet from (20), it oscillates, roughly speaking, between $-\frac{w}{2}$ and $+\frac{w}{2}$. Thus in the above example, the family of $w^{\mathfrak{M}^{*}}$ it is not compact in the strong $L^{q}(\Omega)$ topologies.

\subsection{Discrete $W^{1, p}(\Omega)$ weak compactness}

In relation with Proposition 3.2(ii), let us stress that there is no reason that the components $w^{\mathfrak{M}_{h}}, w^{\mathfrak{M}_{h}^{*}}$ of a sequence $\left(w^{\mathfrak{T}_{h}}\right)_{h}$ of discrete functions with bounded in $L^{p}$ discrete gradients converge to the same limit. Counterexamples are constructed in the same way as in Remark 4 above, starting from two distinct smooth functions discretized, one on the primal mesh $\overline{\mathfrak{M}^{\circ}}$, the other on the dual mesh $\overline{\mathfrak{M}^{*}}$. To cope with this difficulty, the penalization technique of Appendix A can be useful.

The below result shows that in our 3D CeVe-DDFV framework, one should consider that the "true limit" of discrete functions $w^{\mathfrak{T}_{h}}=\left(w^{\mathfrak{M}_{h}^{o}}, w^{\mathfrak{M}_{h}^{*}}\right)$ is the limit of $\frac{1}{3} w^{\mathfrak{M}_{h}^{o}}+\frac{2}{3} w^{\mathfrak{M}_{h}^{*}}$.

Proposition 3.4 (Asymptotic compactness of DDFV discrete functions).

(i) Let $w^{\overline{\boldsymbol{s}}_{h}} \in \mathbb{R}_{0}^{\overline{\boldsymbol{T}}_{h}}$ be discrete functions on a family $\left(\boldsymbol{\mathfrak { T }}_{h}\right)_{h}$ of $3 D$ CeVe-DDFV meshes of $\Omega$ satisfying $\left(M_{\Delta}\right)$, parametrized by $h \geqslant \operatorname{size}\left(\boldsymbol{T}_{h}\right)$. Let us assimilate $w^{\mathfrak{T}_{h}}$ to the piecewise constant functions

$$
w^{\mathfrak{T}_{h}}(x):=\frac{1}{3} w^{\mathfrak{M}^{o} h}+\frac{2}{3} w^{\mathfrak{M}^{*} h}=\frac{1}{3} \sum_{K \in \mathfrak{M}^{o} h} w_{K} 1_{K}(x)+\frac{2}{3} \sum_{K^{*} \in \mathfrak{M}^{*} h} w_{K^{*}} \mathbb{1}_{K^{*}}(x) .
$$

Assume that $\sup _{h \in\left(0, h_{\text {max }}\right]} \operatorname{reg}\left(\boldsymbol{\mathfrak { T }}_{h}\right)<+\infty$, where $\operatorname{reg}\left(\boldsymbol{\mathfrak { T }}_{h}\right)$ measures the regularity of $\boldsymbol{\mathfrak { T }}_{h}$ in the sense (9),(10),(11) and (12). Assume that the family $\left(\nabla^{\mathfrak{T}_{h}} w^{\overline{\mathfrak{s}}_{h}}\right)_{h \in\left(0, h_{\max }\right]}$ is bounded in $L^{p}(\Omega)$ for some $p \in(1,+\infty)$.

Then for all sequence $\left(h_{i}\right)_{i}$ converging to zero there exists $w \in W_{0}^{1, p}(\Omega)$ such that, along a subsequence,

$w^{\mathfrak{T}} h_{i}$ converges to $w=\frac{1}{3} w^{o}+\frac{2}{3} w^{*}$ weakly in $L^{q}(\Omega), q \leqslant p^{*}$

(the components $w^{\mathfrak{M}_{h}^{o}}, w^{\mathfrak{M}_{h}^{*}}$ converge to $w^{o}$, $w^{*}$, resp., strongly in $L^{q}(\Omega), q<p^{*}$ )

and $\nabla^{\mathfrak{T}} h_{i} w^{\overline{\mathfrak{s}}} h_{i}$ converges to $\nabla w$ weakly in $L^{p}(\Omega)$.

(ii) Under the same hypotheses, if $w^{\overline{\boldsymbol{s}}_{h}} \in \mathbb{R}^{\overline{\boldsymbol{x}}_{h}}$ are not assumed to be zero in the boundary volumes, and if the additional assumption of uniform boundedness of

$$
m_{w^{\mathfrak{M}_{h}^{o}}}:=\frac{1}{\operatorname{Vol}(\Omega)} \int_{\Omega} w^{\mathfrak{M}^{o}{ }_{h}}, \quad m_{w^{\mathfrak{M}_{h}^{*}}}:=\frac{1}{\operatorname{Vol}(\Omega)} \int_{\Omega} w^{\mathfrak{M}_{h}^{*}}
$$

is imposed, then (22) holds with $w \in W^{1, p}(\Omega)$. 
(iii) For the meshes satisfying $\left(M_{\square}\right)$, the statement analogous to (i) holds with

$$
\begin{aligned}
w^{\mathfrak{T} h}(x):=\frac{1}{3} w^{\mathfrak{M}_{h}^{o}}+\frac{1}{3} w^{\mathfrak{M}_{\mathfrak{c}, h}^{*}}+\frac{1}{3} w^{\mathfrak{M}_{\mathfrak{b}, h}^{*}} & \\
= & \frac{1}{3} \sum_{K \in \mathfrak{M}_{h}^{o}} w_{K} \mathbb{1}_{K}(x)+\frac{1}{3} \sum_{K^{*} \in \mathfrak{e}_{h}^{*}} w_{K^{*}} 1_{\widehat{K^{*}}}(x)+\frac{1}{3} \sum_{K^{*} \in \mathfrak{o}_{h}^{*}} w_{K^{*}} 1_{\widehat{K^{*}}}(x) ;
\end{aligned}
$$

the strong convergence concerns each of the components $w^{\mathfrak{M}_{h}^{o}}, w^{\mathfrak{M}_{\varepsilon, h}^{*}}, w^{\mathfrak{M}_{6, h}^{*}}$.

The statement analogous to (ii) holds if uniform bounds on the mean values of $w^{\mathfrak{m}^{o}}$ and of $w^{\mathfrak{M}_{\boldsymbol{*}}^{*}}, w^{\mathfrak{M}_{\mathrm{b}}^{*}}$ on $\Omega$ are imposed.

It should be noticed that formula (23) is analogous to the natural reconstruction formula for the 3D CeVEFE-DDFV schemes as considered by Coudière and Hubert in [25] and by Eymard, Guichard and Herbin [46].

Remark 5.

(i) In the case $p=1$, the claim remains true with the limit $w$ that belongs to $B V(\Omega) \cap L^{1^{*}}(\Omega)$, and with discrete gradients converging weakly-* in $B V$ to $\nabla w$.

(ii) The compactness claim for sequences of discrete functions with non-homogeneous boundary conditions on a part of the boundary can be obtained as in [12, Lemma 3.8].

Proof of Proposition 3.4:

Let us prove (i). The strong compactness claim for $\left(w^{\mathfrak{m}_{h}^{o}}\right)_{h}$ and $\left(w^{\mathfrak{M}_{h}^{*}}\right)_{h}$ follows by Proposition 3.2(ii); the weak $L^{p^{*}}$ compactness of $\left(w^{\mathfrak{T}_{h}}\right)_{h}$ comes from Proposition 3.2(i). The weak $L^{p}$ compactness of the family $\left(\nabla^{\mathfrak{x}_{h}} w^{\overline{\mathfrak{x}}_{h}}\right)_{h}$ is immediate from its $L^{p}(\Omega)$ boundedness. Thus if $w$ is the weak $L^{p}$ limit of a sequence $w^{\mathfrak{T}_{h}}=\frac{1}{3} w^{\mathfrak{M}_{h}^{o}}+\frac{2}{3} w^{\mathfrak{M}_{h}^{*}}$ as $h \rightarrow 0$ and $\chi$ is the weak $L^{p}$ limit of the associated sequence of discrete gradients $\nabla^{\mathfrak{T}_{h}} w^{\overline{\mathfrak{x}}_{h}}$, it only remains to show that $\chi=\nabla w$ in the sense of distributions and that $w$ has zero trace on $\partial \Omega$. These two statements follow from the identity

$$
\forall \overrightarrow{\mathcal{F}} \in \mathcal{D}(\bar{\Omega})^{3} \quad \int_{\Omega} \chi \cdot \overrightarrow{\mathcal{F}}+\int_{\Omega} w \operatorname{div} \overrightarrow{\mathcal{F}}=0
$$

that we now prove. We exploit the discrete duality (2) and the consistency property of Proposition (3.1)(i),(iii).

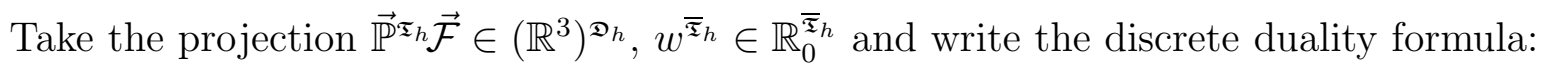

$$
\left.\left\{\left\{\nabla^{\mathfrak{T}_{h}} w^{\overline{\mathfrak{x}}_{h}}, \overrightarrow{\mathbb{P}}^{\mathfrak{\mathfrak { T }}_{h}} \overrightarrow{\mathcal{F}}\right\}\right\}_{\Omega}+\llbracket w^{\mathfrak{T}_{h}}, \operatorname{div}^{\mathfrak{x}_{h}}\left(\overrightarrow{\mathbb{P}}^{\mathfrak{\mathfrak { T }}_{h}} \overrightarrow{\mathcal{F}}\right)\right]_{\Omega}=0 .
$$

According to the definition (4) of $\{\{\cdot, \cdot\}\}_{\Omega}$, the first term in (25) is precisely the integral over $\Omega$ of the scalar product of the constant per diamond fields $\nabla^{\mathfrak{x}_{h}} w^{\overline{\mathfrak{s}}_{h}}$ and $\overrightarrow{\mathbb{P}}^{\mathfrak{x}_{h}} \overrightarrow{\mathcal{F}}$. By Proposition 3.1(i) and the definition of $\chi$, this term converges to the first term in (24) as $h \rightarrow 0$. Similarly, introducing the projection $\mathbb{P}^{\mathfrak{x}_{h}}(\operatorname{div} \overrightarrow{\mathcal{F}})$ of $\operatorname{div} \overrightarrow{\mathcal{F}}$ on $\mathbb{R}^{\mathfrak{x}_{h}}$, from the definition (3) of $[[\cdot, \cdot]]_{\Omega}$, Proposition 3.1(i) and the definition of $w^{\overline{\mathfrak{x}}_{h}}$ in $(21)$ we see that, as $h \rightarrow 0$,

$$
\left.\llbracket\left[w^{\mathfrak{T}_{h}}, \mathbb{P}^{\mathfrak{T}}(\operatorname{div} \overrightarrow{\mathcal{F}})\right]\right]_{\Omega} \longrightarrow \frac{1}{3} \int_{\Omega}\left(\lim _{h \rightarrow 0} w^{\mathfrak{m}^{o}}\right) \operatorname{div} \overrightarrow{\mathcal{F}}+\frac{2}{3} \int_{\Omega}\left(\lim _{h \rightarrow 0} w^{\mathfrak{M}^{*}{ }_{h}}\right) \operatorname{div} \overrightarrow{\mathcal{F}}=\int_{\Omega} w \operatorname{div} \overrightarrow{\mathcal{F}}
$$


It remains to invoke Proposition (3.1)(iii) and the $L^{1}(\Omega)$ bound on $\nabla^{\mathfrak{s}_{h}} w^{\overline{\mathfrak{s}}_{h}}$ to justify the fact that

$$
\lim _{h \rightarrow 0}\left[\left[w^{\mathfrak{T}_{h}}, \operatorname{div}^{\mathfrak{T}_{h}}\left(\overrightarrow{\mathbb{P}}^{\mathfrak{\mathfrak { T }}_{h}} \overrightarrow{\mathcal{F}}\right)\right]_{\Omega}=\lim _{h \rightarrow 0} \llbracket\left[w^{\mathfrak{T}_{h}}, \mathbb{P}^{\mathfrak{T}_{h}}(\operatorname{div} \overrightarrow{\mathcal{F}})\right]_{\Omega} .\right.
$$

For a proof of (ii), one uses the versions of the compact Sobolev embeddings with control by the mean value in $\Omega$, and uses test functions $\overrightarrow{\mathcal{F}}$ compactly supported in $\Omega$.

The point (iii) is shown with the same arguments, using Proposition 3.1(iii-bis) and (20).

\subsection{Discrete operators, functions and fields on $(0, T) \times \Omega$}

Whenever evolution equations are discretized in space with the help of the DDFV operators as described above, analogous consistency properties, Poincaré inequality and discrete $L^{p}\left(0, T ; W^{1, p}(\Omega)\right)$ compactness properties hold.

To be specific, given a CeVe-DDFV mesh $\mathfrak{T}$ of $\Omega$ and a time step $\Delta t$, one considers the additional projection operator

$$
\mathbb{S}^{\Delta t}: f \mapsto\left(f^{n}\right)_{n \in\left[1, N_{\Delta t}\right]} \subset L^{1}(\Omega), \quad f^{n}(x):=\frac{1}{\Delta t} \int_{(n-1) \Delta t}^{n \Delta t} f(t, x) d t .
$$

Here $f$ can mean a function in $L^{1}((0, T) \times \Omega)$ or a field in $\left(L^{1}((0, T) \times \Omega)\right)^{3}$. The smallest integer greater than or equal to $T / \Delta t$ is denoted by $N_{\Delta t}$. It is always meant that $n$ takes values in $[1, T / \Delta t] \cap \mathbb{N}$; in other words, $n$ takes the values $1, \ldots, N_{\Delta t}$.

One defines discrete functions $w^{\mathfrak{T}, \Delta t} \in\left(\mathbb{R}^{\mathfrak{T}}\right)^{N_{\Delta t}}$ on $(0, T) \times \Omega$ as collections of discrete functions $w^{\mathfrak{s}, n}$ on $\Omega$ parametrized by $n \in\left[1, N_{\Delta t}\right]$. Discrete functions $w^{\mathfrak{T}, \Delta t} \in\left(\mathbb{R}^{\overline{\mathfrak{T}}}\right)^{N_{\Delta t}}$ on $(0, T) \times \bar{\Omega}$ and discrete fields $\overrightarrow{\mathcal{F}}^{\mathfrak{T}, \Delta t} \in\left(\left(\mathbb{R}^{3}\right)^{\mathfrak{P}}\right)^{N_{\Delta t}}$ are defined similarly.

The associated norms are defined in a natural way; e.g., the discrete $L^{p}\left(0, T ; W_{0}^{1, p}(\Omega)\right)$ norm of a discrete function $w^{\overline{\mathfrak{s}}, n} \in\left(\mathbb{R}_{0}^{\overline{\mathfrak{x}}}\right)^{N_{\Delta t}}$ is computed as

$$
\sum_{n=1}^{N_{\Delta t}} \Delta t\left\|\nabla^{\mathfrak{T}} w^{\overline{\mathfrak{s}}, n}\right\|_{L^{p}(\Omega)} .
$$

To treat space-time dependent test functions and fields in the way of Proposition 3.1, one replaces the projection operators $\mathbb{P}^{\mathfrak{I}}$ (and its components $\mathbb{P}^{\mathfrak{M}^{o}}, \mathbb{P}^{\mathfrak{M}^{*}}$ ), $\mathbb{P}^{\overline{\mathfrak{I}}}$ and $\overrightarrow{\mathbb{P}}^{\mathfrak{\mathfrak { I }}}$ by their compositions with $\mathbb{S}^{\Delta t}$. Then the statement and the proof of Proposition 3.1 and Corollary 3.1 are extended in a straightforward way.

Also the statement of Proposition 3.4 extends naturally to this time-dependent case; one only has to replace the statement $(22)$ with the weak $L^{p}\left(0, T ; W^{1, p}(\Omega)\right)$ convergence statement:

$w^{\mathfrak{T}_{h}, \Delta t_{h}}$ converges to $w=\frac{1}{3} w^{o}+\frac{2}{3} w^{*}$ weakly in $L^{p}((0, T) \times \Omega)$

(and the components $w^{\mathfrak{M}_{h}^{o}}, w^{\mathfrak{M}_{h}^{*}}$ converge to $w^{o}, w^{*}$, resp., weakly in $L^{p}((0, T) \times \Omega)$ )

and $\nabla^{\mathfrak{T}_{h}} w^{\overline{\mathfrak{s}}_{h}, \Delta t_{h}}$ converges to $\nabla w$ weakly in $L^{p}(\Omega)$

as $\operatorname{size}\left(\boldsymbol{T}_{h}\right)+\Delta t_{h} \rightarrow 0$. It is natural that strong compactness on the space-time cylinder $(0, T) \times \Omega$ does not follow from the sole space discrete gradient bound; one also needs some control of time oscillations. It is also well known that this control can be a very weak one (cf. e.g. the well-known Aubin-Lions and Simon lemmas; see [51] for a discrete version). In the next section, we give the discrete version of a similar result due to Kruzhkov [65]. 


\subsection{Strong compactness in $L^{1}((0, T) \times \Omega)$}

Here we state a lemma that combines a basic space translates estimate (for the "compactness in space") with the Kruzhkov $L^{1}$ time compactness lemma (see [65]). Actually, the Kruzhkov lemma is, by essence, a local compactness result. For the sake of simplicity, we state and prove the version suitable for discrete functions null on the boundary; the general $L_{l o c}^{1}([0, T] \times$ $\Omega$ ) version can be shown with the same arguments (cf. [8]). Other techniques for time compactness of finite volume approximations of evolution equations can be found in [49] and [51]; see also the survey [2].

\section{Proposition 3.5 (Discrete Kruzhkov space-time compactness lemma).}

Let $\left(u^{\overline{\bar{s}}_{h}, \Delta t_{h}}\right)_{h} \in\left(\mathbb{R}_{0}^{\overline{\mathbf{s}}_{h}}\right)^{N_{\Delta t h}}$ be a family of discrete functions on the cylinder $(0, T) \times \Omega$ corresponding to a family $\left(\Delta t_{h}\right)_{h}$ of time steps and to a family $\left(\boldsymbol{T}_{h}\right)_{h}$ of $3 D$ CeVe-DDFV meshes of $\Omega$ satisfying $\left(M_{\Delta}\right)^{7}$; we mean that $h \geqslant \operatorname{size}\left(\boldsymbol{T}_{h}\right)+\Delta t_{h}$.

Assume that $\sup _{h \in\left(0, h_{\text {max }}\right]} \operatorname{reg}\left(\boldsymbol{T}_{h}\right)<+\infty$, where $\operatorname{reg}\left(\boldsymbol{T}_{h}\right)$ measures the regularity of $\boldsymbol{T}_{h}$ in the sense (9) and (10).

For all $h>0$, assume that discrete functions $u^{\overline{\mathfrak{s}}_{h}, \Delta t_{h}}$ satisfy the discrete evolution equations

$$
\text { for } n \in\left[1, N_{\Delta t_{h}}\right], \quad \frac{b\left(u^{\mathfrak{T}_{h}, n}\right)-b\left(u^{\mathfrak{T}_{h}, n-1}\right)}{\Delta t}=\operatorname{div}^{\mathfrak{x}_{h}} \overrightarrow{\mathcal{F}}^{\mathfrak{x}_{h}, n}+f^{\mathfrak{x}_{h}, n}
$$

with some fixed uniformly continuous ${ }^{8}$ non-decreasing function $b: \mathbb{R} \rightarrow \mathbb{R}$, with some initial data $b\left(u^{\mathfrak{T}_{h}, 0}\right) \in \mathbb{R}^{\mathfrak{x}_{h}}$, source terms $f^{\mathfrak{z}_{h}, \Delta t_{h}} \in\left(\mathbb{R}^{\mathfrak{T}_{h}}\right)^{N_{\Delta t h}}$ and discrete fields $\overrightarrow{\mathcal{F}}^{\mathfrak{x}_{h}, \Delta t_{h}} \in$ $\left(\left(\mathbb{R}^{3}\right)^{\mathfrak{D}_{h}}\right)^{N_{\Delta t h}}$.

Assume that there exists a constant $M$ such that the uniform $L^{1}((0, T) \times \Omega)$ estimates hold:

$$
\begin{aligned}
\sum_{n=1}^{N_{\Delta t_{h}}} \Delta t\left(\left\|b\left(u^{\mathfrak{M}^{o}, n}\right)\right\|_{L^{1}(\Omega)}\right. & +\left\|b\left(u^{\mathfrak{M}_{h}^{*}, n}\right)\right\|_{L^{1}(\Omega)} \\
& \left.+\left\|f^{\mathfrak{M}^{o}, n}\right\|_{L^{1}(\Omega)}+\left\|f^{\mathfrak{M}_{h}^{*}, n}\right\|_{L^{1}(\Omega)}+\left\|\overrightarrow{\mathcal{F}}^{\mathfrak{T}_{h}, n}\right\|_{L^{1}(\Omega)}\right) \leqslant M
\end{aligned}
$$

and, moreover, the uniform $L^{1}((0, T) \times \Omega)$ estimate on $\nabla^{\mathfrak{T}_{h}} u^{\mathfrak{T}_{h}, \Delta t_{h}}$ holds:

$$
\sum_{n=1}^{N_{\Delta t_{h}}} \Delta t\left\|\nabla^{\mathfrak{T}_{h}} u^{\mathfrak{T}_{h}, n}\right\|_{L^{1}(\Omega)} \leqslant M
$$

Assume that the families $\left(b\left(u^{\mathfrak{M}_{h}^{o}, 0}\right)\right)_{h},\left(b\left(u^{\mathfrak{M}_{h}^{*}, 0}\right)\right)_{h}$ are bounded in $L^{1}(\Omega)$.

Then for all sequence $\left(h_{i}\right)_{i}$ converging to zero there exist $\beta^{o}, \beta^{*} \in L^{1}((0, T) \times \Omega)$ such that, along a subsequence,

$$
b\left(u^{\mathfrak{M}^{o} h_{i}, \Delta t_{h_{i}}}\right) \longrightarrow \beta^{o} \text { and } b\left(u^{\mathfrak{M}^{*} h_{i}, \Delta t_{h_{i}}}\right) \longrightarrow \beta^{*} \quad \text { in } L^{1}((0, T) \times \Omega) \text { as } i \rightarrow \infty .
$$

Notice that nor the specific structure of the DDFV meshes is important for the above result, neither the dimension; we refer to [8] for a version of the lemma on the admissible finite volume meshes in the sense of [41]. Yet, contrarily to the situation with the Sobolev compact embedding results, in the proof of Proposition 3.5 the two meshes $\mathfrak{M}^{o}, \mathfrak{M}^{*}$ should

7 The proof under assumption $\left(M_{\square}\right)$ is analogous, it requires to replace (30) by slightly different estimates, as in the proof of Prop. 3.3.

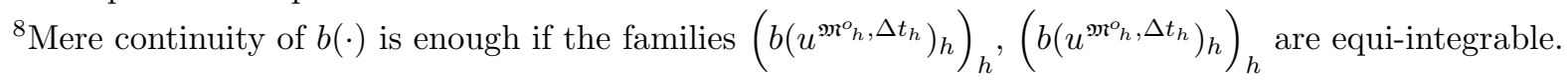


not be considered separately; and the discrete duality (2) is an important ingredient of the proof.

Before turning to the proof, let us mention that in view of the uniform $L^{1}((0, T) \times$ $\Omega$ ) bound on the two components of $b\left(u^{\tau_{h}, \Delta t_{h}}\right)$, the assumption that the components of $\left(b\left(u^{\mathfrak{T}_{h}, 0}\right)\right)_{h}$ are bounded in $L^{1}(\Omega)$ is clearly not essential (yet, it is not restrictive in practice). The only purpose of the assumption that $u^{\overline{\mathfrak{s}}_{h}, \Delta t_{h}}$ are zero in the boundary volumes of $\boldsymbol{T}_{h}$ is to make trivial the issue of extension of $u^{\overline{\mathfrak{s}}_{h}, \Delta t_{h}}$ in space to a small neighborhood of $\Omega$.

To stress the three aspects of the meshing that are important for the below proof, firstly, let us recall that the assumption $l=3$ yields the bounds (17) that we rewrite as

$$
\frac{\left|w_{L}-w_{K}\right|}{d_{K L}} \leqslant\left|\nabla_{\mathrm{S}} w^{\overline{\mathfrak{x}}}\right|, \quad \frac{\left|w_{L^{*}}-w_{K^{*}}\right|}{d_{K^{*} L^{*}}} \leqslant\left|\nabla_{\mathrm{S}} w^{\overline{\mathfrak{x}}}\right|
$$

for $S=S_{K^{*} L^{*}}^{K L}$. Secondly, notice that the construction of the discrete gradient and the assumptions (9) and (10) permit to get the bound

$$
\forall w^{o}, w^{*} \in \mathcal{D}(\Omega) \quad\left\|\nabla^{\mathfrak{T}}\left(\mathbb{P}^{\overline{\mathfrak{M}^{o} h}} w^{o}, \overline{\mathbb{P}^{\overline{\mathfrak{M}_{h}^{*}}}} w^{*}\right)\right\|_{L^{\infty}(\Omega)} \leqslant C(\operatorname{reg}(\mathfrak{T}))\left(\left\|\nabla w^{o}\right\|_{L^{\infty}(\Omega)}+\left\|\nabla w^{*}\right\|_{L^{\infty}(\Omega)}\right) .
$$

Thirdly, note that the discrete duality property readily yields the bound

$$
\left.\forall w^{\overline{\mathfrak{T}}} \in \mathbb{R}_{0}^{\overline{\mathfrak{T}}} \quad \forall \overrightarrow{\mathcal{F}}^{\mathfrak{T}} \in\left(\mathbb{R}^{3}\right)^{\mathfrak{D}} \quad||\left[-\operatorname{div}^{\mathfrak{\mathfrak { T }}} \overrightarrow{\mathcal{F}}^{\mathfrak{T}}, w^{\mathfrak{T}}\right]\right|_{\Omega} \leqslant\left\|\nabla^{\mathfrak{T}} w^{\mathfrak{T}}\right\|_{L^{\infty}(\Omega)}\left\|\overrightarrow{\mathcal{F}}^{\mathfrak{T}}\right\|_{L^{1}(\Omega)} .
$$

\section{Proof of Proposition 3.5:}

The proof is divided into four steps, the heart of it being Step 3.

First, let us fix some notation. Set $Q_{T}:=(0, T) \times \Omega$. We will denote by $u^{h, o}(t, x)$, $u^{h, *}(t, x)$ the components

$$
u^{h, o}(t, x):=\sum_{n=1}^{N_{\Delta t_{h}}} u^{\mathfrak{M}_{h}^{o}, n}(x) \mathbb{1}_{\left((n-1) \Delta t_{h}, n \Delta t_{h}\right]}(t), \quad u^{h, *}(t, x):=\sum_{n=1}^{N_{\Delta t_{h}}} u^{\mathfrak{M}_{h}^{*}, n}(x) \mathbb{1}_{\left((n-1) \Delta t_{h}, n \Delta t_{h}\right]}(t)
$$

of the discrete solution. These functions are extended by the constant in $t$ values $u^{\mathfrak{M}^{o}, N_{\Delta t_{h}}}(x)$, $u^{\mathfrak{M}_{h}^{*}, N_{\Delta t_{h}}}(x)$ on $\left(N_{\Delta t_{h}} \Delta t_{h},+\infty\right)$, then they are extended by zero on $(0,+\infty) \times\left(\mathbb{R}^{3} \backslash \Omega\right)$ (notice that both extensions do not introduce additional jumps).

Step 1: Property (30) and the uniform estimate (29) of the discrete gradient imply the uniform local estimate of the space translates of $u^{h}$ : the space translates of $u^{h, o}$ obey

$$
\sup _{|\Delta x| \leqslant \Delta} \int_{0}^{T} \int_{\mathbb{R}^{3}}\left|u^{h, o}(t, x+\Delta x)-u^{h, o}(t, x)\right| d x d t \leqslant \Delta M C\left(\operatorname{reg}\left(\boldsymbol{T}_{h}\right)\right),
$$

and the identical estimate holds for $u^{h, *}$.

The proof of (33) is standard; we give it here for the sake of completeness. A shorter proof can be derived from the arguments of [42, Lemma 5.1] (it is justified in this lemma that discrete $W^{1,1}$ estimates are in fact $B V$ estimates, and therefore the standard translation properties of the BV functions can be used). 
For $x \in \mathbb{R}^{3}$ and an interface $k \mid L$ of the mesh $\mathfrak{M}_{h}^{o}$, set $\bar{\psi}_{K \mid L}(x)=1$, in case the segment $[x, x+\Delta x]$ crosses $K \mid L$, and $\bar{\psi}_{K \mid L}(x)=0$ otherwise. Note that $\int_{\mathbb{R}^{3}} \bar{\psi}_{K \mid L}(x) d x \leqslant m_{K \mid L} \Delta$. Using (30) and property

$$
\operatorname{reg}\left(\boldsymbol{T}_{h}\right) \operatorname{Vol}\left(s_{K^{*} \mid L^{*}}^{K \mid L}\right) \geqslant m_{K \mid L} d_{K L}
$$

that comes from the inclination bound (10), we have

$$
\begin{gathered}
\int_{0}^{T} \int_{\Omega}\left|u^{h}(t, x)-u^{h}(t, x+\Delta x)\right| d x d t \leqslant \sum_{n=0}^{N_{\Delta t_{h}}} \sum_{\mathrm{S} \in \mathfrak{S}} \Delta t_{h}\left|u_{K}^{n}-u_{L}^{n}\right| \int_{\Omega} \bar{\psi}_{K \mid L}(x) d x \\
\leqslant \Delta \sum_{n=0}^{N_{\Delta t_{h}}} \Delta t_{h} \sum_{S \in \mathfrak{S}} m_{K \mid L}\left|u_{K}^{n}-u_{L}^{n}\right| \leqslant \Delta \sum_{n=0}^{N_{\Delta t_{h}}} \Delta t_{h} \sum_{S \in \mathfrak{S}} m_{K \mid L} d_{K L}\left|\nabla_{\mathfrak{S}} u^{\mathfrak{T}_{h}, n}\right| \\
\leqslant \operatorname{reg}\left(\mathfrak{T}_{h}\right) \Delta \sum_{n=1}^{N_{\Delta t_{h}}} \Delta t_{h} \sum_{S \in \mathfrak{S}} \operatorname{Vol}(S)\left|\nabla_{\mathbb{S}} u^{\mathfrak{T}_{h}, n}\right|
\end{gathered}
$$

meaning as usual that the summation runs over all the subdiamonds $S=S_{K^{*} L^{*}}^{K L}$. The righthand side of the above inequality is exactly $\operatorname{reg}\left(\mathfrak{T}_{h}\right) \Delta \sum_{n=1}^{N_{\Delta t_{h}}} \Delta t_{h}\left\|\nabla^{\mathfrak{T}_{h}} u^{\mathfrak{T}_{h}, n}\right\|_{L^{1}(\Omega)}$, and we conclude using (29).

The same arguments yield the space translation estimate on $u^{h, *}$.

Step 2: We replace the study of $u^{h, o}, u^{h, *}$ (constant per cylinder $Q_{K}^{n}:=K \times\left((n-1) \Delta t_{h}, n \Delta t_{h}\right)$ $\left.\overline{\text { or } Q_{K^{*}}^{n}}:=K^{*} \times\left((n-1) \Delta t_{h}, n \Delta t_{h}\right)\right)$ by the study of functions $\bar{u}^{h, o}, \bar{u}^{h, *}$ continuous in $t$ for all $x$, constant in $x$ for all volume $K$ or $K^{*}$, defined via

$$
\begin{aligned}
b\left(\bar{u}^{h, o}\right)(t, x) & =\sum_{n=1}^{N_{\Delta t_{h}}} \sum_{K \in \mathfrak{M}^{o}{ }_{h}} \frac{1}{\Delta t_{h}}\left(\left(t-(n-1) \Delta t_{h}\right) b\left(u_{K}^{n}\right)+\left(n \Delta t_{h}-t\right) b\left(u_{K}^{n-1}\right)\right) \mathbb{1}_{Q_{K}^{n}}(t, x), \\
\bar{b}\left(u^{h, *}\right)(t, x) & =\sum_{n=1}^{N_{\Delta t_{h}}} \sum_{K^{*} \in \mathfrak{M}^{*}{ }_{h}} \frac{1}{\Delta t_{h}}\left(\left(t-(n-1) \Delta t_{h}\right) b\left(u_{K^{*}}^{n}\right)+\left(n \Delta t_{h}-t\right) b\left(u_{K^{*}}^{n-1}\right)\right) \mathbb{1}_{Q_{K^{*}}^{n}}(t, x) .
\end{aligned}
$$

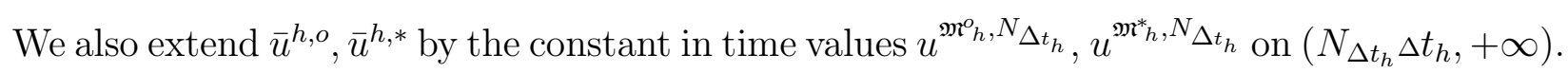

Similarly, we introduce the functions $f^{h}, f^{h, *}$ and $\overrightarrow{\mathcal{F}}^{h}$ in $L^{1}\left(Q_{T}\right)$; moreover, we define

$$
\operatorname{div}^{h, o} \overrightarrow{\mathcal{F}}^{h}:=\sum_{n=1}^{N_{\Delta t_{h}}} \sum_{K \in \mathfrak{M}^{o}{ }_{h}} \operatorname{div}_{K} \overrightarrow{\mathcal{F}}^{\mathfrak{r}, n} 1_{Q_{K}^{n}}(t, x), \quad \operatorname{div}^{h, *} \overrightarrow{\mathcal{F}}^{h}:=\sum_{n=1}^{N_{\Delta t_{h}}} \sum_{K^{*} \in \mathfrak{M}^{*} h} \operatorname{div}_{K^{*}} \overrightarrow{\mathcal{F}}^{\mathfrak{z}, n} 1_{Q_{K^{*}}^{n}}(t, x) .
$$

The functions, $f^{h}, f^{h, *}$ and $\mathcal{F}^{h}, \operatorname{div}^{h, o} \overrightarrow{\mathcal{F}}^{h}, \operatorname{div}^{h, *} \overrightarrow{\mathcal{F}}^{h}$ are extended by zero from $Q_{T}$ to $(0,+\infty) \times$ $\mathbb{R}^{3}$.

Considering the $t$-dependent discrete functions $\bar{u}^{\mathfrak{T}_{h}}(t)$ with the components $\bar{u}^{h, o}(t), \bar{u}^{h, *}(t)$ on the meshes $\mathfrak{M}_{h}^{o}$ and $\mathfrak{M}_{h}^{*}$, we are in a position to rewrite the discrete equations (27) under the form

$$
\frac{\partial}{\partial t} b\left(\bar{u}^{h, o}\right)=\operatorname{div}{ }^{h, o} \overrightarrow{\mathcal{F}}^{h}+f^{h, o}, \quad \frac{\partial}{\partial t} b\left(\bar{u}^{h, *}\right)=\operatorname{div}{ }^{h, *} \overrightarrow{\mathcal{F}}^{h}+f^{h, *}
$$


where the equation is satisfied in $W^{1,1}\left(\mathbb{R}^{+}\right)$in time, for a.e. $x \in \mathbb{R}^{3}$.

In addition, denoting by $\omega_{b}$ a concave modulus of continuity ${ }^{9}$ of $b$, by the definition of $\bar{u}^{h, o}$ and the Jensen inequality we have

$$
\begin{aligned}
& \int_{0}^{+\infty} \int_{\Omega}\left|b\left(\bar{u}^{h, o}\right)(t, x+\Delta x)-b\left(\bar{u}^{h, o}\right)(t, x)\right| d x d t \\
& \quad \leqslant 2 \int_{0}^{T} \int_{\Omega}\left|b\left(u^{h, o}\right)(t, x+\Delta x)-b\left(u^{h, o}\right)(t, x)\right| d x d t+2 \Delta t_{h} \int_{\Omega}\left|b_{0}^{h, o}(x)\right| d x \\
& \quad \leqslant T \operatorname{Vol}(\Omega) \omega_{b}\left(\frac{1}{T \operatorname{Vol}(\Omega)} \int_{0}^{T} \int_{\Omega}\left|u^{h, o}(t, x+\Delta x)-u^{h, o}(t, x)\right| d x d t\right)+2 \Delta t_{h} \int_{\Omega}\left|b_{0}^{h, o}(x)\right| d x
\end{aligned}
$$

where $b_{0}^{h, o}(x)=\sum_{K \in \mathfrak{m}^{o}} b\left(u_{K}^{0}\right) 1_{K}(x)$ is the first component of the initial datum $b\left(u^{\mathfrak{T} h}, 0\right)$.

By the result of Step 1, the assumption $\Delta t_{h} \rightarrow 0$ as $h \rightarrow 0$ and the uniform $L^{1}(\Omega)$ bound on $b\left(u^{\mathfrak{m o}_{h}, 0}\right)_{h}$, the space translates of $\bar{u}^{h, o}$ on $(0, T) \times \Omega$ are estimated uniformly for all sequence $\left(h_{i}\right)_{i}$ convergent to zero. In the same way, the space translates of $\bar{u}^{h, *}$ on $(0, T) \times \Omega$ are estimated. from

Finally, $b\left(\bar{u}^{h_{i}, o}\right), b\left(\bar{u}^{h_{i}, *}\right)$ are bounded in $L^{1}((0, T) \times \Omega)$ uniformly in $i$. Indeed, this comes

$$
\int_{0}^{T} \int_{\Omega}\left|b\left(\bar{u}^{h, o}\right)(t, x)\right| d x d t \leqslant 2 \int_{0}^{\Delta t_{h} N_{\Delta t_{h}}} \int_{\Omega}\left|b\left(u^{h, o}\right)(t, x)\right| d x d t+\Delta t_{h} \int_{\Omega}\left|b_{0}^{h, o}(x)\right| d x
$$

(the identical estimate holds for $b\left(\bar{u}^{h, *}\right)$ ) and from the assumptions of the proposition.

In the sequel, we drop the subscript $i$ in the notation.

Step 3: Now we adapt the idea of the Kruzhkov lemma ([65]). We show that, provided $\bar{u}^{h, o}, \bar{u}^{h, *}$ solve a discrete evolution equation of the form (34) with terms bounded in $L^{1}$ and an estimate of the space translates of $b\left(\bar{u}^{h, o}\right), b\left(\bar{u}^{h, *}\right)$ is available, there is also a uniform estimate of the time translates of $b\left(\bar{u}^{h, o}\right), b\left(\bar{u}^{h, *}\right)$ :

$$
\sup _{\theta \in(0, \tau]} \int_{0}^{+\infty} \int_{\Omega}\left(\frac{1}{3}\left|b\left(\bar{u}^{h, o}\right)(t+\theta, x)-b\left(\bar{u}^{h, o}\right)(t, x)\right|+\frac{2}{3}\left|b\left(\bar{u}^{h, *}\right)(t+\theta, x)-b\left(\bar{u}^{h, *}\right)(t, x)\right|\right) d x d t \leqslant \widetilde{\omega}(\tau) .
$$

Here $\widetilde{\omega}: \mathbb{R}^{+} \longrightarrow \mathbb{R}^{+}$is a modulus of continuity, i.e., a non-decreasing function such that $\lim _{\tau \rightarrow 0} \widetilde{\omega}(\tau)=0$.

Let us construct $\widetilde{\omega}(\cdot)$ verifying $(35)$. First fix $h$ and fix $\theta \in(0, \tau]$. Denote by $I^{h}(\theta)$ the integral in the left-hand side of $(35)$. For $t \geqslant 0$, set

$$
w^{h, o}(t, \cdot):=b\left(\bar{u}^{h, o}\right)(t+\theta, \cdot)-b\left(\bar{u}^{h, o}\right)(t, \cdot), \quad w^{h, *}(t, \cdot):=b\left(\bar{u}^{h, *}\right)(t+\theta, \cdot)-b\left(\bar{u}^{h, *}\right)(t, \cdot) .
$$

Notice that $w^{h, o}(t, \cdot) \equiv 0, w^{h, *}(t, \cdot) \equiv 0$ for large $t$.

Take a standard family $\left(\rho_{\delta}\right)_{\delta}$ of mollifiers on $\mathbb{R}^{3}$ defined as $\rho_{\delta}(x):=\delta^{-3} \rho(x / \delta)$, where $\rho$ is a Lipschitz continuous, nonnegative function supported in the unit ball of $\mathbb{R}^{3}$, and $\int_{\mathbb{R}^{l}} \rho(x) d x=1$. In particular, we have

$$
\left|\nabla \rho_{\delta}\right| \leqslant \frac{C}{\delta^{3+1}}
$$

\footnotetext{
${ }^{9}$ If $b\left(\bar{u}^{h_{i}, o}\right)$ are equi-integrable, a local modulus of continuity is enough.
} 
Here and throughout the proof, $C$ will denote a generic constant independent of $h$ and $\delta$. For all $t>0$, define the function $\varphi^{h, o}(t, \cdot): \mathbb{R}^{3} \longrightarrow \mathbb{R}$ by $\varphi^{h, o}(t):=\rho_{\delta} *\left(\operatorname{sign} w^{h, o}(t)\right)$. In order to lighten the notation, we do not stress the dependence of $\varphi^{h, o}$ on $\delta$. Define $\varphi^{h, *}(t)$ similarly, starting from $w^{h, *}(t)$.

Now discretize $\varphi^{h, o}(t, \cdot)$ on the mesh $\mathfrak{M}_{h}^{o}$ by setting $\varphi^{\mathfrak{M}^{o}}(t):=\mathbb{P}^{\mathfrak{M}^{o}}{ }_{h} \varphi^{h, o}(t, \cdot)$; recall that this means that

$$
\varphi_{K}(t)=\frac{1}{\operatorname{Vol}(K)} \int_{K} \varphi^{h, o}(t, x) d x .
$$

Further, discretize $\varphi^{h, *}(t, \cdot)$ on the mesh $\mathfrak{M}_{h}^{*}$ by setting $\varphi^{\mathfrak{M}^{*}}(t):=\mathbb{P}^{\mathfrak{M}^{*}}{ }_{h} \varphi^{h, *}(t, \cdot)$ Denote by $\varphi^{h}(t)$ the discrete function on the CeVe-DDFV mesh $\boldsymbol{T}_{h}$ of $\Omega$ with the two components $\varphi^{\mathfrak{M}_{h}^{o}}(t), \varphi^{\mathfrak{M}_{h}^{*}}(t)$. Denote by $w^{h}(t)$ the discrete function on $\boldsymbol{T}_{h}$ with the components $w^{h, o}(t), w^{h, *}(t)$.

Now for all fixed $t$, we integrate equations (34) in $t \in[s, s+\theta]$, then take the scalar product $\llbracket \cdot, \cdot \rrbracket_{\Omega}$ of the result by $\varphi^{\mathfrak{T}_{h}}(t)$. Finally, we integrate the obtained equality in $s$ over $\mathbb{R}^{+}$to get

$$
\left.\int_{0}^{+\infty} \llbracket\left[w^{h}(s), \varphi^{h}(s)\right]\right]_{\Omega} d s=\int_{0}^{+\infty} \int_{s}^{s+\theta}\left[\left[\operatorname{div}^{\mathfrak{x}^{h}} \overrightarrow{\mathcal{F}}^{h}(t)+f^{h}(t), \varphi^{h}(s)\right]\right]_{\Omega} d t d s .
$$

Denote by $I_{\delta}^{h}(\theta)$ the left-hand side of (36). Using property (32), the definitions of discrete norms and the Fubini theorem, we infer

$$
\begin{aligned}
I_{\delta}^{h}(\theta) \leqslant \theta( & \left\|\nabla^{\mathfrak{T}} w^{\mathfrak{T}}\right\|_{L^{\infty}(\Omega)}\left\|\mathcal{F}^{h}\right\|_{L^{1}\left(Q_{T}\right)} \\
& \left.+\max \left\{\left\|\varphi^{\mathfrak{M}_{h}^{o}}\right\|_{L^{\infty}\left(Q_{T}\right)},\left\|\varphi^{\mathfrak{M}^{o}}\right\|_{L^{\infty}\left(Q_{T}\right)}\right\} \times\left(\left\|f^{h, o}\right\|_{L^{1}\left(Q_{T}\right)}+\left\|f^{h, *}\right\|_{L^{1}\left(Q_{T}\right)}\right)\right) .
\end{aligned}
$$

Now the $L^{1}([0, T] \times \Omega)$ bounds $(28)$ on $\left(\mathcal{F}^{h}\right)_{h},\left(f^{h, o}\right)_{h}$ and $\left(f^{h, *}\right)_{h}$, the bounds

$$
\left|\varphi^{h, o}(t, \cdot)\right| \leqslant 1,\left|\varphi^{h, *}(t, \cdot)\right| \leqslant 1, \quad\left|\nabla \varphi^{h, o}(t, \cdot)\right| \leqslant C / \delta^{4},\left|\nabla \varphi^{h, *}(t, \cdot)\right| \leqslant C / \delta^{4}
$$

and property (31) yield the estimate

$$
I_{\delta}^{h}(\Delta t) \leqslant \theta C\left(\operatorname{reg}\left(\boldsymbol{T}_{h}\right)\right) M\left(1+\delta^{-4}\right)
$$

for all $h$ and $\delta$ small enough, uniformly in $h$. Now, notice that, $w^{h, o}$ and $\varphi^{h, o}$ being constant per $K \in \mathfrak{M}_{h}^{o}$, by the definition of $\varphi_{K}(t)$ we have

$$
\begin{aligned}
\operatorname{Vol}(K)\left(\left|w_{K}(t)\right|-w_{K}(t) \varphi_{K}(t)\right)= & \operatorname{Vol}(K)\left|w^{h, o}(t, x)\right|-w_{K}(t) \int_{K} \varphi^{h, o}(t, x) d x \\
& =\int_{K}\left(\left|w^{h, o}(t, x)\right|-w^{h, o}(t, x) \varphi^{h, o}(t, x)\right) d x
\end{aligned}
$$

the identical equality holds on dual volumes $K^{*}$. Therefore (recalling again the definition of $\left.\llbracket \cdot, \cdot \rrbracket_{\Omega}\right)$

$$
\begin{aligned}
& I^{h}(\theta)-I_{\delta}^{h}(\theta)=\int_{0}^{+\infty} \int_{\Omega}\left(\frac{1}{3}\left(\left|w^{h, o}(t, x)\right|-w^{h, o}(t, x) \varphi^{h, o}(t, x)\right)\right. \\
&+\left.\frac{2}{3}\left(\left|w^{h, *}(t, x)\right|-w^{h, *}(t, x) \varphi^{h, *}(t, x)\right)\right) d x d t
\end{aligned}
$$


Starting from this point, the argument of Kruzhkov [65] applies exactly as for the "continuous" case. Note the key inequality, valid for all monotone $b$ such that $b(0)=0$ :

$$
\forall \alpha, \gamma \in \mathbb{R} \quad|| \alpha|-\alpha \operatorname{sign} \gamma| \leqslant 2|\alpha-\gamma|
$$

Setting $\sigma:=(x-y) / \delta$, we upper bound $\left|I^{h}(\theta)-I_{\delta}^{h}(\theta)\right|$ by

$$
\begin{aligned}
& 2 \int_{0}^{+\infty} \int_{\Omega} \int_{\mathbb{R}^{3}} \rho_{\delta}(x-y)\left(\frac{1}{3}\left|w^{h, o}(t, x)-w^{h, o}(t, y)\right|+\frac{2}{3}\left|w^{h, *}(t, x)-w^{h, *}(t, y)\right|\right) d y d x d t \leqslant \\
& \leqslant \frac{2}{3} \int_{\mathbb{R}^{3}} \rho(\sigma) \int_{0}^{+\infty} \int_{\Omega}\left(\left|b\left(\bar{u}^{h, o}\right)(t, x)-b\left(\bar{u}^{h, o}\right)(t, x-\delta \sigma)\right|+\left|b\left(\bar{u}^{h, *}\right)(t, x)-b\left(\bar{u}^{h, *}\right)(t, x-\delta \sigma)\right|\right) d x d t d \sigma
\end{aligned}
$$

therefore if $\omega(\cdot)$ is the modulus of continuity controlling the space translates of $\bar{b}\left(u^{h, o}\right)$ and of $\bar{b}\left(u^{h, *}\right)$ in $L^{1}((0, T) \times \Omega)$, then

$$
\left|I^{h}(\theta)-I_{\delta}^{h}(\theta)\right| \leqslant 2 \omega(\delta)
$$

Recall that, by Steps 1 and 2 of the proof, one can choose $\omega(\cdot)$ independent of $h$. Combining (37) with (38), we conclude that the function

$$
\widetilde{\omega}(\tau):=\inf _{\delta>0} C\left\{\tau\left(1+\delta^{-4}\right)+2 \omega(\delta)\right\}
$$

upper bounds the quantity $\sup _{\theta \in(0, \tau]} I^{h}(\theta)$. Because $\widetilde{\omega}(\tau)$ tends to 0 as $\tau \rightarrow 0$, (35) follows. Step 4: By the Frechet-Kolmogorov compactness criterion, the relative compactness of

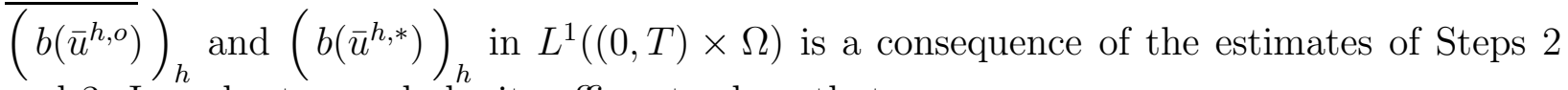
and 3. In order to conclude, it suffices to show that

$$
\left\|b\left(u^{h, o}\right)-b\left(\bar{u}^{h, o}\right)\right\|_{L^{1}((0, T) \times \Omega)} \rightarrow 0 \text { and }\left\|b\left(u^{h, *}\right)-b\left(\bar{u}^{h, *}\right)\right\|_{L^{1}((0, T) \times \Omega)} \rightarrow 0
$$

as $h \rightarrow 0$. An easy calculation shows that

$$
\text { for all } \alpha, \gamma \in \mathbb{R}, \int_{0}^{1}|\kappa \alpha+(1-\kappa) \gamma| d \kappa \geqslant \frac{1}{2}(|\alpha|+|\gamma|) \text {. }
$$

Applying this inequality to $\alpha:=b\left(u^{\mathfrak{M}_{h}^{o}, n+1}\right)-b\left(u^{\mathfrak{M}_{h}^{o}, n}\right), \gamma:=b\left(u^{\mathfrak{M}_{h}^{o}, n}\right)-b\left(u^{\mathfrak{M}_{h}^{o}, n-1}\right)$, from the definition of $\bar{u}^{h}$ we deduce

$$
\int_{0}^{T} \int_{\Omega}\left|b\left(u^{h, o}\right)(t, x)-b\left(\bar{u}^{h, o}\right)(t, x)\right| d x d t \leqslant 2 \int_{0}^{T+\Delta t_{h}} \int_{\Omega}\left|b\left(\bar{u}^{h, o}\right)\left(t+\Delta t_{h}, x\right)-b\left(\bar{u}^{h, o}\right)(t, x)\right| d x d t
$$

The identical estimate holds with $b\left(u^{h, o}\right), b\left(\bar{u}^{h, o}\right)$ replaced by $b\left(u^{h, *}\right), b\left(\bar{u}^{h, *}\right)$. Since $\Delta t_{h}$ tends to zero as $h \rightarrow 0$, estimate (35) of Step 3 implies that the right-hand side of the above inequality converges to zero as $h$ tends to zero. This ends the proof of the proposition. 


\section{CeVe-DDFV scheme for a degenerate parabolic equation and its convergence}

Equation (1) combines two different kinds of degeneracy. In the present paper, we are mainly concerned with one of the difficulties, which is the elliptic degeneracy. It appears, e.g., in the celebrated Richards model for flows in porous media. We refer to [47, 40, 13] and references therein for previous works on this topic. Two other difficulties related to finite volume approximation of the general triply nonlinear equation (1) were treated in [4] and [15].

When $b(\cdot)$ is constant on some interval of $\mathbb{R},(1)$ is an elliptic-parabolic problem. Here, the notion of a variational solution (following Alt and Luckhaus [1]) is enough to establish the well-posedness theory; but some technical difficulties appear, such as time compactness of sequences of solutions. The problem of time compactness is particularly delicate if the "structure condition"

$$
b(z)=b(\hat{z}) \Rightarrow F(z)=F(\hat{z})
$$

is not satisfied (see Bénilan and Wittbold [18]). In Section 4.1, we focus on the case where $A=\mathrm{Id}$ (thus the hyperbolic degeneracy is avoided) and where $F=0$ (thus (39) is trivially satisfied). Convergence of finite volume approximations in the case where (39) fails is treated in [15] using hints that are very different from those presented in the present paper.

On the other hand, when $b(\cdot)$ is constant on some interval of $\mathbb{R}$, equation (1) exhibits hyperbolic features. In particular, the notion of entropy solution is needed to achieve a well-posedness theory. Analysis of the finite volume scheme is particularly involved in this case, it uses entropy techniques in addition to the variational techniques of Section 4.1. In Section 4.2 , we only recall the key ingredient of [4] on convergence of the $3 \mathrm{D}$ CeVe-DDFV scheme to the entropy solution in the case $b=\mathrm{Id}$.

Our main goal in this section is to illustrate the results and techniques of Section 3 and to prove convergence of 3D CeVe-DDFV approximations for the elliptic-parabolic case of the general triply nonlinear scalar equation (1). The role of the weak formulation of the scheme (coming from the discrete duality property) in the convergence proof is emphasized.

Notice that the analytic techniques needed for different kinds of degeneracy in equation (1) can be combined (see in particular [6]). We expect that convergence of finite volume schemes for this general equation can be proved by a careful combination of the arguments of $[6,4,15]$ and of Section 4.1 .

\subsection{Degenerate parabolic problems with Leray-Lions nonlinear diffusion}

Following Alt and Luckhaus [1] (see also [71]), we consider the problem

$$
\left\{\begin{array}{l}
b(u)_{t}=\operatorname{div} \varphi(\nabla u)+f \\
u_{(0, T) \times \partial \Omega}=0, \quad b(u)_{t=0}=b_{0},
\end{array}\right.
$$

for $b: \mathbb{R} \rightarrow \mathbb{R}$ a continuous non-decreasing function with $b(0)=0$, and for

$$
-\operatorname{div} \varphi(\nabla \cdot): W_{0}^{1, p}(\Omega) \rightarrow W^{-1, p^{\prime}}(\Omega)
$$

which is a Leray-Lions operator; namely,

$$
\left\{\begin{array}{l}
\varphi \in C\left(\mathbb{R}^{3}, \mathbb{R}^{3}\right), \quad \forall \xi \neq \eta(\varphi(\xi)-\varphi(\eta)) \cdot(\xi-\eta)>0 \\
\forall \xi \varphi(\xi) \cdot \xi \geqslant c|\xi|^{p}, \quad|\varphi(\xi)|^{p^{\prime}} \leqslant C\left(1+|\xi|^{p}\right)
\end{array}\right.
$$


with $p \in(1,+\infty)$ and $p^{\prime}$ the conjugate exponent of $p$. Due to the non-strict monotonicity of $b$ and the possible degeneracy/singularity of the diffusion coefficient $\varphi^{\prime}(\nabla u)$, problem (40) is a degenerate parabolic problem (for $b \equiv 0$ and $f$ constant in $t$, it reduces to an elliptic problem).

We consider $f \in L^{p^{\prime}}(Q)$ the so-called "finite energy initial data" $b_{0}$, i.e.,

$$
b_{0}: \Omega \mapsto \text { Range }(b), \quad b_{0}=b\left(u_{0}\right) \text { with the restriction } B\left(u_{0}\right)=\int_{0}^{u_{0}} s d b(s) \in L^{1}(\Omega) .
$$

Finite volume discretization of (40) in the parabolic case and its convergence were studied in [13]. Different finite volume schemes and their convergence in the elliptic case were studied in $[9,12,37,43]$; error estimates were obtained, in three different situations, in $[9,10,11]$. Important refinements of the 2D DDFV scheme for the case with discontinuous coefficients were introduced in [20].

Let us consider the following time-implicit DDFV scheme for (40):

$$
\begin{aligned}
& \text { find } u^{\mathfrak{T}, \Delta t}=\left(u^{\mathfrak{\mathfrak { T }}, n}\right)_{n=1 . . N_{\Delta t} \in \mathbb{R}_{0}^{\overline{\mathfrak{T}}} \text { such that }} \\
& \forall n=1 . . N_{\Delta t} \quad \frac{b\left(u^{\mathfrak{T}, n}\right)-b\left(u^{\mathfrak{T}, n-1}\right)}{\Delta t}=\operatorname{div}^{\mathfrak{\mathfrak { x }}} \varphi\left(\nabla^{\mathfrak{T}} u^{\overline{\mathfrak{s}}, n}\right)+f^{\mathfrak{T}, n}+\mathcal{P}^{\mathfrak{T}}\left[u^{\overline{\mathfrak{I}}, n}\right]
\end{aligned}
$$

with the initial conditions and source term given by

$$
b\left(u^{\mathfrak{T}, 0}\right)=\mathbb{P}^{\mathfrak{T}} b_{0}, \quad f^{\mathfrak{T}, n}=\mathbb{P}^{\mathfrak{T}}\left(\mathbb{S}^{\Delta t} f\right)^{n} .
$$

The notation is the one of Sections 2 and 3.5, except for the penalization operator $\mathcal{P}^{\mathfrak{T}}[\cdot]$ introduced and explained in Appendix B. The penalization term serves for the convergence proof; in practice the numerical scheme without this term converges as well. Penalization can also be replaced by a more intricate approximation of the time evolution term; see Remark 6 in Appendix C.

The scheme thus leads to a nonlinear system to be solved at each time step; for a description of the strategy for solving the resulting nonlinear algebraic equations, see [20] and Appendix D.

Proposition 4.1 (Weak discrete formulation of the scheme). A discrete function $u^{\mathfrak{\tau}, \Delta t}$ solves (42) if and only if there holds the weak formulation:

$$
\begin{aligned}
& \forall n=1 . . N_{\Delta t} \quad \forall v^{\mathfrak{T}} \in \mathbb{R}_{0}^{\overline{\mathfrak{T}}} \\
& \left.\left.\llbracket \frac{b\left(u^{\mathfrak{T}, n}\right)-b\left(u^{\mathfrak{T}, n-1}\right)}{\Delta t}, v^{\mathfrak{T}}\right]_{\Omega}+\left\{\left\{\varphi\left(\nabla^{\mathfrak{T}} u^{\overline{\mathfrak{s}}, n}\right), \nabla^{\mathfrak{T}} v^{\overline{\mathfrak{T}}}\right\}\right\}_{\Omega}=\llbracket f^{\mathfrak{T}, n}, v^{\mathfrak{T}}\right]_{\Omega}+\llbracket\left[\mathcal{P}^{\mathfrak{T}} u^{\overline{\mathfrak{s}}, n}, v^{\mathfrak{T}}\right]_{\Omega} .
\end{aligned}
$$

Formulation (42) with the initial condition implies the following weak space-and-time formulation:

$$
\begin{aligned}
& -\sum_{n=0}^{N_{\Delta t}-1} \Delta t\left[\left[b\left(u^{\mathfrak{s}, n}\right), \frac{v^{\mathfrak{T}, n+1}-v^{\mathfrak{T}, n}}{\Delta t}\right]\right]_{\Omega}+\sum_{n=1}^{N_{\Delta t}} \Delta t\left\{\left\{\varphi\left(\nabla^{\mathfrak{T}} u^{\overline{\mathfrak{s}}, n}\right), \nabla^{\mathfrak{T}} v^{\overline{\mathfrak{s}}, n}\right\}\right\}_{\Omega} \\
& =\sum_{n=1}^{N_{\Delta t}} \Delta t\left[f^{\mathfrak{\tau}, n}, v^{\mathfrak{\tau}, n}\right]_{\Omega}+\llbracket\left[b\left(u^{\mathfrak{\tau}, 0}\right), v^{\mathfrak{\tau}, 0}\right]_{\Omega}+\sum_{n=1}^{N_{\Delta t}} \Delta t\left[\left[\mathcal{P}^{\mathfrak{\tau}} u^{\overline{\mathfrak{z}}, n}, v^{\mathfrak{\tau}, n}\right]_{\Omega}\right.
\end{aligned}
$$

for all test function $v^{\mathfrak{s}, \Delta t}$ such that $v^{\mathfrak{T}, N}=0$. 
For the proof, the deduction of $(44),(45)$ from (42) is straightforward using the discrete duality, Proposition 6.1 and (for getting (45)) using the Abel transformation of the sum in $n$. Conversely, to get from (44) to (42) one uses the test functions $v^{\mathfrak{z}}$ with only one non-zero entry.

Theorem 4.1 (Convergence of the scheme (42)). Assume that $b, b_{0}$ and $\varphi$ satisfy the assumptions of the beginning of this section. Let $\boldsymbol{T}$ be a CeVe-DDFV mesh satisfying $\left(M_{\Delta}\right)$ or $\left(M_{\square}\right)$. Let $\Delta t>0$ be a time step. There exists a unique solution to scheme $(42),(43)$.

Further, assume we are given a family $\left(\Delta t_{h}\right)_{h}$ of time steps and a family $\left(\boldsymbol{\mathfrak { T }}_{h}\right)_{h}$ of CeVeDDFV meshes (with $\Delta t_{h}+\operatorname{size}\left(\boldsymbol{\mathfrak { T }}_{h}\right) \leqslant h$ ) satisfying the uniform regularity assumptions (9),(10),(11) and (12). Let $u^{\mathfrak{T}_{h}, \Delta t_{h}}$ denote the corresponding discrete solution, and $\nabla^{\mathfrak{T}} u^{\overline{\mathfrak{T}}_{h}, \Delta t_{h}}$ denote the corresponding discrete gradient of the solution (both are considered as functions of $Q)$. Then as $h \rightarrow 0$, there holds

$$
b\left(u^{\mathfrak{T}_{h}, \Delta t_{h}}\right) \rightarrow b(u) \text { in } L^{1}(Q), \quad u^{\mathfrak{T}_{h}, \Delta t_{h}} \rightarrow u \text { in } L^{p}(Q), \quad \text { and } \nabla^{\mathfrak{T}} u^{\overline{\mathfrak{T}}_{h}, \Delta t_{h}} \rightarrow \nabla u \text { in } L^{p}(Q),
$$

where $u \in L^{p}\left(0, T ; W_{0}^{1, p}(\Omega)\right)$ is the unique solution of $(40)$.

Now we turn to the proof of Theorem 4.1. The proof is not specific to dimension three, the same result and proof apply for the 2D DDFV schemes (cf. [4]). The weak formulations (44),(45) (coming from the discrete duality) are the main tool, along with the "discrete versions" of the arguments of Alt and Luckhaus [1].

ProOF T: emporarily (as long as $h$ is fixed), we drop the subscript $h$ from the notation $\Delta t_{h}$ and $\boldsymbol{T}_{h}$. Uniqueness of a discrete solution follows from the monotonicity of $b(\cdot), \varphi(\cdot)$. Indeed, by induction in $n$, we obtain the equality

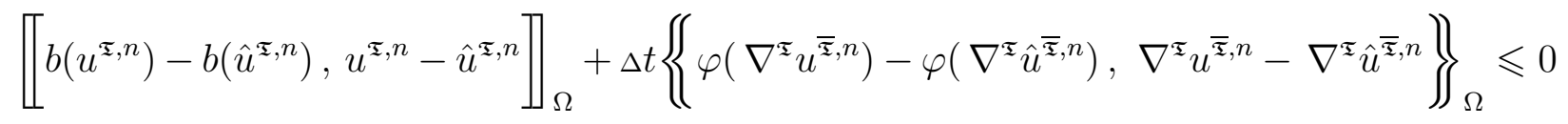

(here $u^{\mathfrak{s}, \Delta t}, \hat{u}^{\mathfrak{s}, \Delta t}$ are two discrete solutions, and we have used (44) at time step $n$ with the test function $v^{\mathfrak{T}}=u^{\mathfrak{T}, n}-\hat{u}^{\mathfrak{T}, n}$; the inequality " $\leqslant$ " comes from the penalization term which is dropped, because it is non-negative). The strict monotonicity of $\varphi(\cdot)$ implies that $\nabla^{\mathfrak{s}} u^{\overline{\mathfrak{s}}, n}=$ $\nabla^{\mathfrak{T}} \hat{u}^{\overline{\mathfrak{s}}, n}$, whence $u^{\overline{\mathfrak{s}}, n}=\hat{u}^{\overline{\mathfrak{s}}, n}$ from the discrete Poincaré inequality of Proposition 3.2(ii) or Proposition 3.3.

Further, existence of a discrete solution follows by an application of the Brouwer fixedpoint theorem or of the finite-dimensional topological degree theory from the a priori estimates we now prove (see e.g. $[13,12,37,43]$ ). Indeed, first note the following convexity inequality:

$$
(b(z)-b(\hat{z})) z \geqslant B(z)-B(\hat{z}) \text { with } B(z)=\int_{0}^{z} s d b(s) .
$$

Assume $u^{\mathfrak{T}, \Delta t}$ is a solution of (42),(43). Using $v^{\mathfrak{T}}=u^{\mathfrak{T}, n}$ as test function in formulation (44) at time step $n$, summing in $n$ from 1 to $k, k \leqslant N_{\Delta t}$ and using (46) for the time evolution terms, the coercivity (41) in the diffusion term and the Young and discrete Poincaré inequalities in the source term, we find the a priori bound

$$
\begin{aligned}
\max _{1 \leqslant k \leqslant N_{\Delta t}}\left(\sum_{K} m_{K} B\left(u_{K}\right)+\right. & \left.\sum_{K^{*}} m_{K^{*}} B\left(u_{K^{*}}\right)\right)+\sum_{n=1}^{N_{\Delta t}} \Delta t \sum_{D} m_{D}\left|\nabla_{D} u^{\overline{\mathfrak{s}}, n}\right|^{p} \\
& +\sum_{n=1}^{N_{\Delta t}} \Delta t \llbracket\left[\mathcal{P}^{\mathfrak{s}} u^{\overline{\mathfrak{s}}, n}, u^{\overline{\mathfrak{s}}, n}\right]_{\Omega} \leqslant \operatorname{const}\left(\|f\|_{L^{p^{\prime}(Q)}}^{p^{\prime}}+\left\|B\left(u_{0}\right)\right\|_{L^{1}(\Omega)}\right) .
\end{aligned}
$$


Notice that in the variational case, i.e., for $\varphi=\nabla \Phi$, the discrete duality to show (see e.g. [12], note also Proposition 6.1) that the discrete solution $u^{\mathfrak{T}, n}$ at time step $n$ is the unique minimizer of the following convex coercive functional:

$$
\begin{aligned}
J^{n}\left[u^{\mathfrak{T}}\right]:= & \frac{1}{3} \sum_{K} m_{K} D\left(u_{K}\right)+\frac{2}{3} \sum_{K^{*}} m_{K^{*}} D\left(u_{K^{*}}\right)+\Delta t \sum_{D} m_{D} \Phi\left(\nabla_{D} u^{\overline{\mathfrak{T}}}\right) \\
& \left.\left.-\llbracket \Delta t f^{\mathfrak{T}, n}+b\left(u^{\mathfrak{T}, n-1}\right), u^{\mathfrak{T}}\right]\right]_{\Omega}+\frac{1}{3} \sum_{K \in \overline{\mathfrak{M}^{o}}, K^{*} \in \overline{\mathfrak{M}^{*}}} \operatorname{Vol}\left(K \cap K^{*}\right) \frac{\left(u_{K}-u_{K^{*}}\right)^{2}}{\operatorname{size}(\mathfrak{T})},
\end{aligned}
$$

where $D(\cdot)$ is a primitive of $b(\cdot)$. This variational point of view may be useful for a practical implementation of the nonlinear scheme using descent algorithms (cf. [9, 12]); the coordination-decomposition approach of [54] is a more general and more efficient alternative, see [20]. We recall this algorithm in Appendix C. Notice that the Newton method may fail because of the degeneracy of $b(\cdot)$.

Now, estimate (47) contains, in particular, a uniform $L^{p}(Q)$ bound on the discrete functions $\nabla^{\mathfrak{T}_{h}} u^{\overline{\mathfrak{T}}_{h}, \Delta t_{h}}$. Then the compactness result (26) permits to extract a subsequence (here and in the sequel, extracted subsequences are not relabelled) such that

$$
\nabla^{\mathfrak{T}_{h}} u^{\overline{\mathfrak{s}}_{h}, \Delta t_{h}} \rightarrow \nabla u \text { in } L^{p}(Q) \text { weakly with } u \in L^{p}\left(0, T ; W_{0}^{1, p}(\Omega)\right)
$$

and $u:=\frac{1}{3} u^{o}+\frac{2}{3} u^{*}$ with $u^{\mathfrak{M}_{h}^{o}, \Delta t_{h}} \rightarrow u^{o}$ and $u^{\mathfrak{M}_{h}^{*}, \Delta t_{h}} \rightarrow u^{*}$ in $L^{p}(Q)$ weakly

(the lack of control of time oscillations precludes us from getting the strong convergence here). Moreover, Proposition 6.2 means that $u^{o}=u^{*}=u$. From the growth assumption in (41) we infer the convergence $\varphi\left(\nabla^{\mathfrak{s}_{h}} u^{\overline{\mathfrak{z}}_{h}, \Delta t_{h}}\right) \rightarrow \chi$ weakly in $L^{p^{\prime}}(Q)$. Furthermore, the $L^{1}(Q)$ estimate of $\nabla^{\mathfrak{T}_{h}} u^{\overline{\mathfrak{T}}_{h}, \Delta t_{h}}$, the discrete evolution equations (42) and the discrete Kruzhkov lemma (Proposition 3.5) permit to get the strong convergences

$$
b\left(u^{\mathfrak{M}_{h}^{o}, \Delta t_{h}}\right) \rightarrow \beta^{o} \text { and } b\left(u^{\mathfrak{M}_{h}^{*}, \Delta t_{h}}\right) \rightarrow \beta^{*} \text { in } L^{1}(Q)
$$

(notice that (47) and the definition of $B(\cdot)$ imply equi-integrability of $b\left(u^{\mathfrak{M}_{h}^{o}, \Delta t_{h}}\right), b\left(u^{\mathfrak{M}^{*}}{ }_{h}, \Delta t_{h}\right)$, so that mere continuity of $b(\cdot)$ is enough). The monotonicity of $b(\cdot)$ permits to identify both $\beta^{o}$ and $\beta^{*}$ with $b(u)$, using the equi-integrability of $\left(u^{\mathfrak{M}_{h}^{o}, \Delta t_{h}}\right)_{h},\left(u^{\mathfrak{M}_{h}^{*}, \Delta t_{h}}\right)_{h}$ and the Minty argument. Consequently, we also have the strong convergence

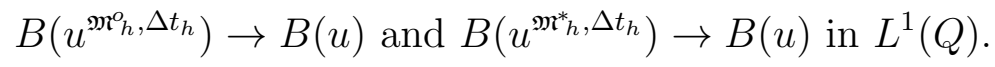

Without loss of restriction, we may assume that the above convergence also takes place in $L^{1}(\Omega)$ for $t=T$ (this takes place for a.e. $T>0$ ). Now, we can pass to the limit in the weak discrete formulation (45) (using, in particular, Corollary 3.1 for the initial condition term, using the parabolic version of Corollary 3.1 for the source term, and using the parabolic analogue of Proposition 3.1 for the test function) to get

$$
-\iint_{Q} b(u) v_{t}-\int_{\Omega} b_{0} v(0, \cdot)+\iint_{Q} \chi \cdot \nabla v=\iint_{Q} f v
$$

with regular test functions $v$ that are zero at $t=T$. Then, in the way of Alt and Luckhaus [1] we can write (48) under the equivalent "variational" formulation with test functions 
$v \in X=L^{p}\left(0, T ; W_{0}^{1, p}(\Omega)\right)$, using the duality product to give sense to the product of $v$ by $b(u)_{t} \in X^{*}=L^{p^{\prime}}\left(0, T ; W^{-1, p^{\prime}}(\Omega)\right)$ :

$$
-\iint_{Q} b(u) v_{t}-\int_{\Omega} b_{0} v(0, \cdot) \text { becomes } \int_{0}^{T}<b(u)_{t}, v>_{W^{-1, p^{\prime}}, W_{0}^{1, p}} .
$$

The key step is to identify $\chi$ (the weak limit of $\chi^{\mathfrak{T}_{h}, \Delta t_{h}}:=\varphi\left(\nabla^{\mathfrak{T}_{h}} u^{\overline{\mathfrak{s}}_{h}, \Delta t_{h}}\right)$ ) with $\varphi(\nabla u)$ (see, e.g., [1]). This is done starting from the weak formulation (44), with $v^{\mathfrak{T}_{h}}=u^{\mathfrak{T}_{h}, n}$ at the time step $n$ : summing in $n$ from 1 to $N_{\Delta t_{h}}$ and using the convexity inequality (46), we get

$$
\begin{aligned}
\frac{1}{3} \sum_{K} m_{K} B\left(u_{K}^{N_{\Delta t_{h}}}\right)+\frac{2}{3} \sum_{K^{*}} m_{K^{*}} B\left(u_{K^{*}}^{N_{\Delta t_{h}}}\right)+\sum_{n=1}^{N_{\Delta t_{h}}} \Delta t_{h}\left\{\left\{\varphi\left(\nabla^{\mathfrak{\mathfrak { T }}_{h}} u^{\overline{\mathfrak{T}}_{h}, \Delta t_{h}}\right), \nabla^{\mathfrak{\mathfrak { T }}_{h}} u^{\overline{\mathfrak{T}}_{h}, \Delta t_{h}}\right\}\right\}_{\Omega} \\
\leqslant \sum_{n=1} \Delta t_{h}\left[\left[f^{\mathfrak{T}_{h}, n}, u^{\mathfrak{T}_{h}, n}\right]\right]_{\Omega}+\frac{1}{3} \sum_{K} m_{K} B\left(u_{K}^{0}\right)+\frac{2}{3} \sum_{K^{*}} m_{K^{*}} B\left(u_{K^{*}}^{0}\right) .
\end{aligned}
$$

Notice that the penalization term has the good sign and it is dropped in the above inequality. Using Corollary 3.1 and the continuity of $B(z)$ as function of $b(z)$ (for the initial condition term) and using the parabolic version of Corollary 3.1 (for the source term), from the previously obtained weak convergences we deduce

$$
\lim _{h \rightarrow 0} \sum_{n=1}^{N_{\Delta t_{h}}} \Delta t_{h}\left\{\left\{\varphi\left(\nabla^{\mathfrak{s}_{h}} u^{\overline{\mathfrak{s}}_{h}, \Delta t_{h}}\right), \nabla^{\mathfrak{s}_{h}} u^{\overline{\mathfrak{s}}_{h}, \Delta t_{h}}\right\}\right\}_{\Omega} \geqslant \iint_{Q} f u-\int_{\Omega}\left(B(u(T))-B\left(u_{0}\right)\right) ;
$$

at this point, we have used that $B\left(u^{o}\right) \equiv B\left(u^{*}\right) \equiv B(u)$. By the integration-by-parts argument of $[1,71]$,

$$
\int_{\Omega}\left(B(u(T))-B\left(u_{0}\right)\right)=\int_{0}^{T}<b(u)_{t}, u>_{W^{-1, p^{\prime}}, W_{0}^{1, p}} .
$$

Thus the right-hand side of (49) can be written as

$$
\iint_{Q} f u-\int_{0}^{T}<b(u)_{t}, u>_{W^{-1, p^{\prime}}, W_{0}^{1, p}} \text { and then, due to (48), as } \iint_{Q} \chi \cdot \nabla u .
$$

Hence we get the inequality

$$
\iint_{Q} \chi \cdot G \geqslant \liminf _{h \rightarrow 0} \sum_{n=1}^{N_{\Delta t_{h}}} \Delta t_{h}\left\{\left\{\varphi\left(\nabla^{\mathfrak{T}_{h}} u^{\overline{\mathfrak{x}}_{h}, \Delta t_{h}}\right), \nabla^{\mathfrak{x}_{h}} u^{\overline{\mathfrak{s}}_{h}, \Delta t_{h}}\right\}\right\}_{\Omega}=\liminf _{h \rightarrow 0} \varphi\left(G_{h}\right) \cdot G_{h}
$$

with $G=\nabla u$ and $G_{h}=\nabla^{\mathfrak{T}_{h}} u^{\overline{\mathfrak{z}}_{h}, \Delta t_{h}}$. With (50) in hand, the monotonicity of $\varphi(\cdot)$ in (41) and the classical Minty-Browder argument (see e.g. [1]; cf. [12, 37, 43] for its use in finite volumes) ensures that $\chi=\varphi(G)$ and, moreover, the strict monotonicity of $\varphi(\cdot)$ yields strong convergence in $L^{p}(Q)$ of $G_{h}$ to $G$.

With $\chi=\varphi(\nabla u)$, we see that (48) is indeed the weak formulation of problem (40); thus $u$ is a solution of the problem. The uniqueness of a solution permits to get the convergence results as $h \rightarrow 0$ without extracting subsequences.

More general problems of kind (40) can be discretized and their convergence can be proved in much the same spirit; for the elliptic case, see [12, 20, 43] and [37] for the cases of $x$-dependent and $u$-dependent Leray-Lions type nonlinearities $\varphi(x, u, \nabla u)$, respectively. 


\subsection{Degenerate convection-diffusion problems and discrete entropy dissipation}

The DDFV schemes were initially designed as one among many other solutions to the problem of approximating anisotropic diffusion problems or even isotropic problems on general meshes (Hermeline [57, 56, 59, 60], Domelevo and Omnès [36], Pierre et al. [72, 32]). They turned out to be well suited for nonlinear diffusion problems ([12, 20, 26] and the above Section 4.1). The common feature here is that these problems are analyzed using variational techniques, i.e. using the solution itself as the test function. The discrete duality feature permits to get energy conservation properties that lead to a priori estimates; with the estimates (and thus the weak compactness properties) in hand, one recasts the scheme under the weak form (see Proposition 4.1 in Section 4.1; cf. [7]) and then proves convergence.

Many important applications require the use of nonlinear test functions. To be specific, we focus on the equation (1) in the degenerate parabolic-hyperbolic case, i.e., $b=\mathrm{Id}$ and $F \neq 0$, i.e.,

$$
u_{t}-\operatorname{div}(\varphi(\nabla A(u))-F(u))=f
$$

with $A$ continuous, increasing but non-strictly increasing, and with $\varphi(\cdot)$ of the kind (41). In this context, the convergence analysis requires the use of nonlinear test functions and of dissipation inequalities in the place of energy conservation identities. The same is true for linear problems analyzed by nonlinear methods, such as generalized solutions of $-\Delta u=$ $f$ with $L^{1}$ or measure datum $f$ (see Droniou, Gallouët et Herbin [39],[50] and references therein). In both cases, the analysis methods heavily rely on nonlinear chain rule arguments that are not natural in the discrete setting.

While the DDFV discretization of the diffusion term appears as suitable in both cases (and on quite general meshes, as it was the case in Section 4.1), the tools of stability and convergence analysis seem to require the orthogonality condition on the meshes, in order to get appropriate "chain rule kind"-inequalities. Indeed, in the previous works on the subject the condition $\overrightarrow{x_{K} x_{L}} \perp K \mid L$ was always exploited (see [50,39] and the conclusion of the survey paper [38] for the linear case; see [44,4] for the case $(51))$. The schemes of $[50,39,44]$ are two-point schemes in the spirit of Eymard, Gallouët and Herbin [41] (the diffusion being linear and isotropic in these cases); while in [4], the diffusion is nonlinear and therefore, an "orthogonal" DDFV scheme, in $3 D$ and in $2 D$, was used. The scheme takes the form

$$
\begin{aligned}
& \text { find } u^{\mathfrak{T}, \Delta t}=\left(u^{\mathfrak{T}, n}\right)_{n=1 . . N_{\Delta t}} \in \mathbb{R}_{0}^{\overline{\mathfrak{T}}} \text { such that } \\
& \forall n=1 . . N_{\Delta t} \quad \frac{u^{\mathfrak{T}, n}-u^{\mathfrak{T}, n-1}}{\Delta t}+\operatorname{divc}^{\mathfrak{T}} F\left(u^{\overline{\mathfrak{T}}, n}\right)=\operatorname{div}^{\mathfrak{T}} \varphi\left(\nabla^{\mathfrak{T}} A\left(u^{\overline{\mathfrak{z}}, n}\right)\right)+\mathcal{P}^{\mathfrak{T}}\left[u^{\overline{\mathfrak{z}}, n}\right]
\end{aligned}
$$

where $u^{\mathfrak{T}} \mapsto \operatorname{divc}^{\mathfrak{T}} F\left(u^{\mathfrak{T}}\right)$ is a discrete convection operator (approximation of $u \mapsto \operatorname{div} F(u)$ ) using standard two-point monotone consistent flux approximation (see $[41,44,4]$ ).

Let us point out the key ingredient of the convergence analysis of [4], which can be seen as a discrete entropy dissipation inequality for diffusion terms. The analogous entropy dissipation inequality for convection terms is well known $([44,4])$. Notice that a discrete maximum principle for DDFV approximations of (51) is deduced from this result.

Proposition 4.2 (Chain rule inequality, see [4]).

Let $\boldsymbol{T}$ de a DDFV mesh in $2 D$ or in $3 D$; in the latter case, assume all the primal interfaces $\kappa \mid L$ are triangles. Impose the following orthogonality restriction:

for all neighbors $K, L$ (resp., $\left.K^{*}, L^{*}\right)$ there holds $\overrightarrow{x_{K} x_{L}} \perp K \mid L$ (resp., $\left.\overrightarrow{x_{K^{*}} x_{L^{*}}} \perp K^{*} L^{*}\right)$, 
and assume that $\varphi(\xi)=k(|\xi|) \xi$ with a nonnegative (possibly singular at zero) function $k(\cdot)$. Let $A$ be a continuous nondecreasing function on $\mathbb{R}$; given a non-decreasing function $\theta$ on $\mathbb{R}$, set $A_{\theta}(z)=\int_{0}^{z} \theta(s) d A(s)$.

Let $u^{\overline{\mathbf{s}}} \in \mathbb{R}_{0}^{\overline{\mathbf{\Sigma}}}$ and $\psi^{\mathfrak{\tau}} \in \mathbb{R}_{0}^{\overline{\mathbf{\Sigma}}}$ (the zero boundary condition for $\psi^{\mathfrak{\tau}}$ can be replaced by the requirement $\theta(0)=0$ ), with $\psi^{\mathfrak{T}} \geqslant 0$. Then the following "dissipative chain rule property" holds:

$$
\begin{aligned}
\left.\llbracket \operatorname{div}^{\mathfrak{T}}\left[k\left(\nabla^{\mathfrak{T}} A\left(u^{\overline{\mathfrak{T}}}\right)\right) \nabla^{\mathfrak{T}} A\left(u^{\overline{\mathfrak{T}}}\right)\right], \theta\left(u^{\mathfrak{T}}\right) \psi^{\mathfrak{T}}\right]_{\Omega} \\
\leqslant-\left\{\left\{k\left(\nabla^{\mathfrak{T}} A\left(u^{\overline{\mathfrak{T}}}\right)\right) \nabla^{\mathfrak{T}} A_{\theta}\left(u^{\overline{\mathfrak{T}}}\right), \nabla^{\mathfrak{T}} \psi^{\overline{\mathfrak{T}}}\right\}\right\}_{\Omega} .
\end{aligned}
$$

Inequality (53) comes from the convexity inequality that replaces the chain rule $A^{\prime}(z) \theta(z)=$ $\left(A_{\theta}\right)^{\prime}(z)$ :

$$
(A(z)-A(\hat{z})) \theta(\hat{z}) \leqslant A_{\theta}(z)-A_{\theta}(\hat{z}) \quad \text { for all } z, \hat{z} \in \mathbb{R} .
$$

The proof is straightforward: it uses the summation-by-parts procedure, the particular structure $\varphi(\xi)=k(|\xi|) \xi$ of $\varphi(\cdot)$ which is, in particular, isotropic, and also the particular expression of the discrete gradient under the orthogonality condition that avoids mixing the primal and the dual unknowns (except in the term $k\left(\nabla^{\mathfrak{T}} A\left(u^{\overline{\mathfrak{z}}}\right)\right)$ that is not transformed). In absence of the orthogonality condition (or for anisotropic $\varphi$ ) we are not aware of any proof of properties that could play the role of (53) in the convergence analysis for (51).

\section{Numerical results for elliptic-parabolic problems}

We illustrate the convergence behavior of the CeVe-DDFV scheme for the parabolic problem (40) in two situations. Test 1 corresponds to a linear anisotropic problem, whereas Test 2 is a fully non linear one.

\section{Test 1}

$$
\begin{aligned}
b & =I d \\
\varphi(\xi) & =A \xi, \text { with } A=\left(\begin{array}{ccc}
1 & 0.5 & 0 \\
0.5 & 1 & 0 \\
0 & 0.5 & 1
\end{array}\right)
\end{aligned}
$$

\section{Test 2}

$$
b(s)=\frac{1}{2}(1-\cos (\pi s)) \mathbb{1}_{[0,1]}(s)+\mathbb{1}_{[1,+\infty[}(s)
$$$$
\varphi(\xi)=|\xi|^{p-2} \xi \text {, with } p=3
$$

The tests are performed in the 3D unit square, on three families of meshes: cubic meshes, tetrahedral meshes and prismatic meshes with general faces as illustrated in Figure 3. We
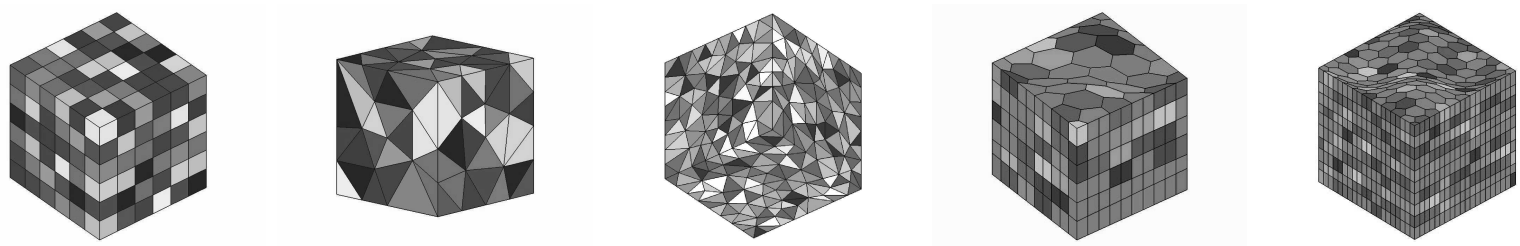

Figure 3. Cubic mesh level $0(6 \times 6 \times 6)$, Tetrahedral meshes level 0 and level 1 , Prismatic meshes level 0 $(5 \times 5 \times 5)$ and level $1(10 \times 10 \times 10)$

take the exact solution $u(t, x)=v(t) w(x, y, z)=e^{-t} \sin (\pi x) \sin (\pi y) \sin (\pi z)$ (now $(x, y, z)$ 


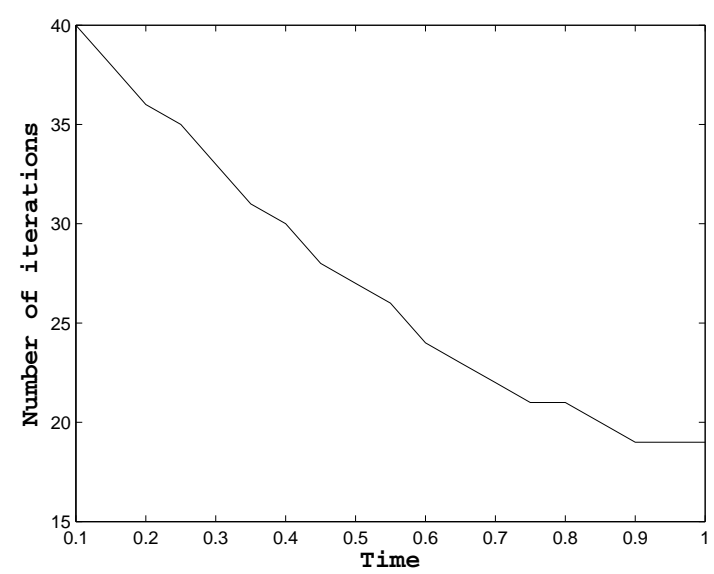

Figure 4. Number of iterations of the decomposition coordination algorithm for Test 2 (Prismatic mesh level 0 is used).

denotes a generic point of the unit cube), with the homogeneous Dirichlet boundary condition (note that $\left.w\right|_{\partial \Omega}=0$ ), and we calculate the source term corresponding to the solution $u$ of (40). The penalization operator used in the convergence proof of Section 4.1, is omitted. In case of Test 2, the scheme is nonlinear; it is solved thanks to the decomposition-coordination algorithm described in Appendix D.

Behavior of the decomposition-coordination algorithm. We fixed here the value of the penalization parameters $r$ and $\gamma$ to 1. Optimal choice of this parameters will not be investigated here. The number of iterations of the decomposition coordination decreases with time, as it is shown in Figure 4 (with the tolerance tol that has been fixed to $10^{-5}$ ). Note that no more than three iterations are needed in the Newton steps of the algorithm.

Accuracy of the scheme. To put the discrete and the exact solutions "at the same level", we use the projection $\mathbb{P}^{\sqrt{s}} u_{e}$ of the exact solution and the associated discrete gradient reconstruction $\overrightarrow{\nabla^{\mathfrak{I}}} \mathbb{P}^{\overline{\mathfrak{s}}} u_{e}$. The $L^{p}\left(0 . T ; L^{p}(\Omega)\right)$ norm of the error $e^{\mathfrak{T}}:=u^{\mathfrak{T}}-\mathbb{P}^{\mathfrak{s}} u_{e}(p=2$ for Test 1$)$, as well as the $L^{\infty}\left(0 . T ; L^{p}(\Omega)\right)$ norm of the gradient $\overrightarrow{\nabla^{\mathfrak{\Sigma}}} e^{\bar{\tau}}:=\vec{\nabla}^{\mathfrak{\Sigma}} u^{\overline{\mathfrak{s}}}-\vec{\nabla}^{\mathfrak{\Sigma}} \mathbb{P}^{\bar{\Sigma}} u_{e}$ and the $L^{\infty}\left(0 . T ; L^{1}(\Omega)\right)$ of $\delta^{\mathfrak{T}} b=b\left(u^{\mathfrak{T}}\right)-b\left(\mathbb{P}^{\mathfrak{T}} u_{e}\right)$ are reported in Tables $1-5$. We focus here on the accuracy of the diffusive part of the discrete operator. Therefore we have adapted the time step $\Delta t$ to the size of the mesh by choosing $\left.\Delta t_{i}=\Delta t_{0}\left((\# \text { unknowns })_{i} /(\# \text { unknowns })_{0}\right)\right)^{\frac{1}{3}}$. Note that we obtain super-convergence for cubic meshes even in the nonlinear case; this was observed for other kinds of schemes (see, e.g., [11]).

\section{B. Appendix A: A penalization operator}

Penalization of a DDFV scheme may be useful in order to guarantee that the two components of a discrete "double" function $w^{\mathfrak{T}_{h}}$ converge to the same limit.

Indeed, we have seen that in the context of Proposition 3.4, the two components $w^{\mathfrak{m}^{o} h}$, $w^{\mathfrak{M}_{h}^{*}}$ of discrete functions $w^{\mathfrak{T}_{h}}$ with bounded discrete $W_{0}^{1, p}$ norm may converge to two distinct limits. This can complicate the analysis of the DDFV discretizations for certain PDEs (although the fact that the components $w^{\mathfrak{M}_{h}^{o}}, w^{\mathfrak{M}_{h}^{*}}$ converge to a common limit can be 


\begin{tabular}{|c|c|c|c|c|c|c|c|}
\hline Mesh level & $\Delta t$ & $\left\|\tilde{e}^{\mathfrak{T}}\right\|_{L^{p}\left(L^{p}\right)}$ & rate & $\left\|\overrightarrow{\nabla^{\mathfrak{T}}} \overline{e^{\bar{T}}}\right\|_{L^{p}\left(L^{p}\right)}$ & rate & $\left\|\delta^{\mathfrak{T}} b\right\|_{L^{\infty}\left(L^{1}\right)}$ & rate \\
\hline $0-6 \times 6 \times 6$ & $5.0 \mathrm{E}-02$ & $0.455 \mathrm{E}-01$ & - & $0.520 \mathrm{E}-01$ & - & $0.390 \mathrm{E}-01$ & - \\
\hline $1-9 \times 9 \times 9$ & $2.4 \mathrm{E}-02$ & $0.198 \mathrm{E}-01$ & 1.933 & $0.228 \mathrm{E}-01$ & 1.918 & $0.174 \mathrm{E}-01$ & 1.878 \\
\hline $2-12 \times 12 \times 12$ & $1.25 \mathrm{E}-02$ & $0.111 \mathrm{E}-01$ & 1.938 & $0.127 \mathrm{E}-01$ & 1.931 & $0.979 \mathrm{E}-02$ & 1.905 \\
\hline $3-18 \times 18 \times 18$ & $5.07 \mathrm{E}-03$ & $0.488 \mathrm{E}-02$ & 1.951 & $0.564 \mathrm{E}-02$ & 1.947 & $0.436 \mathrm{E}-02$ & 1.933 \\
\hline $4-24 \times 24 \times 24$ & $2.79 \mathrm{E}-03$ & $0.274 \mathrm{E}-02$ & 1.962 & $0.317 \mathrm{E}-02$ & 1.960 & $0.245 \mathrm{E}-02$ & 1.953 \\
\hline
\end{tabular}

Table 1. Test 1-Cubic meshes

\begin{tabular}{|c|c|c|c|c|c|c|c|}
\hline Mesh level & $\Delta t$ & $\left\|\tilde{e}^{\mathfrak{I}}\right\|_{L^{p}\left(L^{p}\right)}$ & rate & $\left\|\overrightarrow{\nabla^{\mathfrak{\tau}}} \bar{e}^{\bar{x}}\right\|_{L^{p}\left(L^{p}\right)}$ & rate & $\left\|\delta^{\mathfrak{T}} b\right\|_{L^{\infty}\left(L^{1}\right)}$ & rate \\
\hline 0 & $5.0 \mathrm{E}-02$ & $0.856 \mathrm{E}-01$ & - & $0.164 \mathrm{E}+00$ & - & $0.774 \mathrm{E}-01$ & - \\
\hline 1 & $1.16 \mathrm{E}-02$ & $0.187 \mathrm{E}-01$ & 1.998 & $0.685 \mathrm{E}-01$ & 1.148 & $0.154 \mathrm{E}-01$ & 2.122 \\
\hline 2 & $7.02 \mathrm{E}-03$ & $0.117 \mathrm{E}-01$ & 2.072 & $0.535 \mathrm{E}-01$ & 1.098 & $0.103 \mathrm{E}-01$ & 1.799 \\
\hline 3 & $4.42 \mathrm{E}-03$ & $0.742 \mathrm{E}-02$ & 1.987 & $0.396 \mathrm{E}-01$ & 1.308 & $0.671 \mathrm{E}-02$ & 1.850 \\
\hline 4 & $2.79 \mathrm{E}-03$ & $0.485 \mathrm{E}-02$ & 1.852 & $0.313 \mathrm{E}-01$ & 1.015 & $0.445 \mathrm{E}-02$ & 1.786 \\
\hline
\end{tabular}

Table 2. Test 1-Tetrahedral meshes

\begin{tabular}{|c|c|c|c|c|c|c|c|}
\hline Mesh & $\Delta t$ & $\left\|\tilde{e}^{\mathfrak{T}}\right\|_{L^{p}\left(L^{p}\right)}$ & rate & $\left\|\overrightarrow{\nabla^{\mathfrak{T}}} \overline{\tilde{e}}\right\|_{L^{p}\left(L^{p}\right)}$ & rate & $\left\|\delta^{\mathfrak{T}} b\right\|_{L^{\infty}\left(L^{1}\right)}$ & rate \\
\hline $0-5 \times 5 \times 5$ & $5.0 \mathrm{E}-02$ & $0.462 \mathrm{E}-01$ & - & $0.584 \mathrm{E}-01$ & - & $0.418 \mathrm{E}-01$ & - \\
\hline $1-10 \times 10 \times 10$ & $1.51 \mathrm{E}-02$ & $0.143 \mathrm{E}-01$ & 1.886 & $0.202 \mathrm{E}-01$ & 1.710 & $0.134 \mathrm{E}-01$ & 1.831 \\
\hline $2-15 \times 15 \times 15$ & $6.18 \mathrm{E}-03$ & $0.631 \mathrm{E}-02$ & 1.916 & $0.101 \mathrm{E}-01$ & 1.617 & $0.595 \mathrm{E}-02$ & 1.895 \\
\hline
\end{tabular}

Table 3. Test 1-Prismatic meshes with general faces

\begin{tabular}{|c|c|c|c|c|c|c|c|}
\hline $\mathrm{Me}$ & $\Delta t$ & $\left\|e^{\mathfrak{T}}\right\|_{L^{p}\left(L^{p}\right)}$ & rate & $\left\|\overrightarrow{\nabla^{\mathfrak{T}} \overline{e^{\mathfrak{x}}}}\right\|_{L^{p}\left(L^{p}\right)}$ & rate & $\left\|\delta^{\mathfrak{T}} b\right\|_{L^{\infty}\left(L^{1}\right)}$ & rate \\
\hline $0-$ & $\overline{0 \mathrm{E}-0}$ & $0.456 \mathrm{E}-01$ & - & $0.497 \mathrm{E}-01$ & & $0.525 \mathrm{E}-01$ & - \\
\hline $1-9 x 9 x 9$ & $2.4 \mathrm{E}-02$ & $0.19^{\prime}$ & .950 & $0.217 \mathrm{E}-01$ & .921 & $0.222 \mathrm{~F}$ & 1.993 \\
\hline $2-12 \times 12 \times 12$ & $.25 \mathrm{E}-02$ & $0.110 \mathrm{E}-01$ & 1.942 & $0.122 \mathrm{E}-01$ & 1.934 & 0.123 & 1.976 \\
\hline $3-18 \times 18 \times 18$ & $5.07 \mathrm{E}-03$ & $0.485 \mathrm{E}-02$ & 1.952 & $0.539 \mathrm{E}-02$ & 1.943 & $0.539 \mathrm{E}-02$ & 1.965 \\
\hline
\end{tabular}

Table 4. Test 2 : Cubic meshes

\begin{tabular}{|c|c|c|c|c|c|c|c|}
\hline Mesh level & $\Delta t$ & $\left\|\tilde{e}^{\mathfrak{T}}\right\|_{L^{p}\left(L^{p}\right)}$ & rate & $\left\|\overrightarrow{\nabla^{\mathfrak{T}}} \overline{\tilde{\widetilde{x}}}\right\|_{L^{p}\left(L^{p}\right)}$ & rate & $\left\|\delta^{\mathfrak{I}} b\right\|_{L^{\infty}\left(L^{1}\right)}$ & rate \\
\hline $0-5 \times 5 \times 5$ & $5.0 \mathrm{E}-02$ & $0.472 \mathrm{E}-01$ & - & $0.588 \mathrm{E}-01$ & - & $0.593 \mathrm{E}-01$ & - \\
\hline $1-10 \times 10 \times 10$ & $1.51 \mathrm{E}-02$ & $0.143 \mathrm{E}-01$ & 1.922 & $0.214 \mathrm{E}-01$ & 1.626 & $0.175 \mathrm{E}-01$ & 1.961 \\
\hline $2-15 \times 15 \times 15$ & $6.18 \mathrm{E}-03$ & $0.620 \mathrm{E}-02$ & 1.950 & $0.115 \mathrm{E}-01$ & 1.456 & $0.765 \mathrm{E}-02$ & 1.939 \\
\hline
\end{tabular}

Table 5. Test 2-Prismatic meshes with general faces 
implied by the structure of the PDE considered; see [12] for one particular case). In the context of the work [4], this difficulty turned out to be an obstacle for proving convergence of the DDFV scheme. Therefore the idea to penalize the differences $w_{K}-w_{K^{*}}$ for $K \cap K^{*} \neq \varnothing$ was introduced. Let us give some details.

On the set $\mathbb{R}^{\overline{\mathfrak{z}}}$ of discrete functions $w^{\overline{\mathfrak{s}}}$ on $\bar{\Omega}$, following [4] we define the operator $\mathcal{P}^{\mathfrak{s}}[\cdot]$ of "double" mesh penalization by

$$
\mathcal{P}^{\mathfrak{T}}: w^{\mathfrak{T}} \in \mathbb{R}^{\mathfrak{T}} \mapsto \mathcal{P}^{\mathfrak{T}} w^{\mathfrak{T}}=\left(\left(\mathcal{P}_{K} w^{\mathfrak{T}}\right)_{K \in \mathfrak{M}^{\circ}},\left(\mathcal{P}_{K^{*}} w^{\mathfrak{T}}\right)_{K^{*} \in \mathfrak{M}^{*}}\right) \in \mathbb{R}^{\mathfrak{T}}
$$

where the entries $\mathcal{P}_{K} w^{\mathfrak{T}}, \mathcal{P}_{K^{*}} w^{\mathfrak{T}}$ of the discrete function $\mathcal{P}^{\mathfrak{T}} w^{\mathfrak{T}}$ on $\Omega$ are given by

$$
\begin{aligned}
\mathcal{P}_{K} w^{\mathfrak{T}} & :=2 \frac{1}{\operatorname{size}(\mathfrak{T})} \frac{1}{\operatorname{Vol}(K)} \sum_{K^{*} \in \overline{\mathfrak{M}^{*}}} \operatorname{Vol}\left(K \cap K^{*}\right)\left(w_{K}-w_{K^{*}}\right) ; \\
\mathcal{P}_{K^{*}} w^{\mathfrak{T}} & :=\frac{1}{\operatorname{size}(\mathfrak{T})} \frac{1}{\operatorname{Vol}\left(K^{*}\right)} \sum_{K \in \overline{\mathfrak{M}^{o}}} \operatorname{Vol}\left(K \cap K^{*}\right)\left(w_{K^{*}}-w_{K}\right) .
\end{aligned}
$$

The definitions are designed to get the following summation-by-parts formula:

Proposition 6.1 (Summation-by-parts for penalization operator). Let $w^{\overline{\mathfrak{s}}} \in \mathbb{R}^{\overline{\mathfrak{z}}}$ and $\varphi^{\overline{\mathfrak{s}}} \in \mathbb{R}_{0}^{\overline{\mathfrak{x}}}$. Then

$$
\left.\llbracket \mathcal{P}^{\mathfrak{T}} w^{\overline{\mathfrak{T}}}, \varphi^{\mathfrak{T}}\right]_{\Omega}=\frac{2}{3} \sum_{K \in \overline{\mathfrak{M}^{o}}, K^{*} \in \overline{\mathfrak{M}^{*}}} \operatorname{Vol}\left(K \cap K^{*}\right) \frac{\left(w_{K}-w_{K^{*}}\right)\left(\varphi_{K}-\varphi_{K^{*}}\right)}{\operatorname{size}(\mathfrak{T})} .
$$

The proof is straightforward from the definitions of $\llbracket \cdot, \cdot \rrbracket_{\Omega}$ in $(3)$ and of the operator $\mathcal{P}^{\mathfrak{x}}$.

Notice that adding such a penalization term into discrete equations corresponds, roughly speaking, to adding a small amount of discrete diffusion (e.g. on the uniform Cartesian DDFV meshes of Section 2.1, the penalization operator is in fact a size $(\boldsymbol{T})$-small multiple of a discrete Laplacian). Therefore it is clear that this additional term does not affect the convergence of the schemes. In addition, except in some degenerate situations (e.g. on the meshes satisfying the orthogonality condition, the equations corresponding to primal and dual volumes are actually not coupled) adding this term does not enlarge the stencil of the scheme.

Adding the penalization leads to an additional estimate on discrete solutions that can be used as follows.

Proposition 6.2 (Compactness in presence of the penalization operator). In the assumptions of Proposition 3.4, let us require in addition that

$$
\llbracket\left[\mathcal{P}^{\mathfrak{x}_{h}} w^{\overline{\mathfrak{s}_{h}}}, w^{\mathfrak{T}_{h}}\right]_{\Omega} \leqslant \text { const }
$$

uniformly in $h$. Then (upon extraction of convergent subsequences) the limits of the families $\left(w^{\mathfrak{M}^{o}}{ }_{h}\right.$ and $\left(w^{\mathfrak{M}_{h}^{*}}\right)_{h}$ coincide.

The proof is straightforward (see [4]).

In a similar manner, for the case of Cartesian meshes one could penalize the differences between the neighbor values on $\kappa^{*} \in \mathfrak{e}_{h}^{*}$ and on $\kappa^{*} \in \mathfrak{o}_{h}^{*}$, to ensure that $w^{\mathfrak{M}_{*}^{*}, h}$ and $w^{\mathfrak{M}_{,}^{*}, h}$ converge to the same limit (notice that in case of such penalization, the example of Remark 4 would not be compatible with the uniform estimate (54)). 


\section{C. Appendix B: DDFV discretization of reaction terms}

Recall that (unless the penalization technique of the previous paragraph is used) the two components $w^{\mathfrak{M}_{h}^{o}}, w^{\mathfrak{M}_{h}^{*}}$ of discrete functions $w^{\mathfrak{T}_{h}}$ may converge to two distinct limits $w^{o}, w^{*}$. Therefore, discretizing a function $\Psi:=\psi(w)$ on $\mathfrak{T}$ in the most straightforward way, namely

$$
\Psi^{\mathfrak{T}}:=\left(\left(\psi\left(w_{K}\right)\right)_{K \in \mathfrak{M}^{\circ}},\left(\psi\left(w_{K^{*}}\right)\right)_{K^{*} \in \mathfrak{M}^{*}}\right)
$$

may lead to a difficulty. Indeed, at the limit e.g. of $\left.I_{h}:=\llbracket(\psi(w))^{\mathfrak{T}_{h}}, w^{\mathfrak{\tau}_{h}}\right]_{\Omega}$ we will find (see the definition (3) of the scalar product on $\mathbb{R}^{\mathfrak{T}}$ )

$$
\int_{\Omega}\left(\frac{1}{3} \psi\left(w^{o}\right) w^{o}+\frac{2}{3} \psi\left(w^{*}\right) w^{*}\right)
$$

Because we have seen that the function $\frac{1}{3} w^{o}+\frac{2}{3} w^{*}$ should be considered as the natural limit of $w^{\mathfrak{T}_{h}}$ (namely, $\nabla^{\mathfrak{T}_{h}} w^{\overline{\mathfrak{x}}_{h}}$ converges to the gradient of this function), as long as we cannot prove that $w^{o}=w^{*}$ we need to find

$$
I:=\int_{\Omega} \psi\left(\frac{1}{3} w^{o}+\frac{2}{3} w^{*}\right)\left(\frac{1}{3} w^{o}+\frac{2}{3} w^{*}\right) .
$$

at the limit of $I_{h}$, in the place of (55). Therefore we suggest that either penalization operators (see Appendix A) be used in order to enforce the equality $w^{o}=w^{*}$; or that the reaction terms be discretized on a 3D CeVe-DDFV mesh by taking, for discretization of $\Psi=\psi(w)$, the expression

$$
\begin{gathered}
\Psi^{\mathfrak{T}}:=\left(\left(\psi\left(\check{w}_{K}\right)\right)_{K \in \mathfrak{M}^{o}},\left(\psi\left(\check{w}_{K^{*}}\right)\right)_{K^{*} \in \mathfrak{M}^{*}}\right), \\
\check{w}_{K}:=\frac{1}{3} w^{K}+\frac{2}{3} \sum_{K^{*} \in \overline{\mathfrak{M}^{*}}} \frac{\operatorname{Vol}\left(K \cap K^{*}\right)}{\operatorname{Vol}(K)} w_{K^{*}}, \quad \check{w}_{K^{*}}:=\frac{1}{3} \sum_{K \in \overline{\mathfrak{M}^{*}}} \frac{\operatorname{Vol}\left(K \cap K^{*}\right)}{\operatorname{Vol}\left(K^{*}\right)} w^{K}+\frac{2}{3} w_{K^{*}}
\end{gathered}
$$

In other words, $\check{w}_{K}$ and $\check{w}_{K^{*}}$ are the mean values of the function $\frac{1}{3} w^{\mathfrak{M}^{o}}+\frac{2}{3} w^{\mathfrak{M}^{*}}$ on $K$ and on $K^{*}$, respectively. With this choice, we have for all $w^{\mathfrak{T}} \in \mathbb{R}_{0}^{\overline{\mathfrak{T}}}$, for all $\varphi^{\mathfrak{T}} \in \mathbb{R}^{\mathfrak{T}}$,

$$
\left.\llbracket\left[(\psi(w))^{\mathfrak{T}}, \varphi^{\mathfrak{T}}\right]\right]_{\Omega}=\int_{\Omega} \psi\left(\frac{1}{3} w^{\mathfrak{M}^{o}}+\frac{2}{3} w^{\mathfrak{M}^{*}}\right)\left(\frac{1}{3} \varphi^{\mathfrak{M}^{o}}+\frac{2}{3} \varphi^{\mathfrak{M}^{*}}\right) .
$$

Notice that in the case of general meshes, such treatment of reaction terms does not enlarge the stencil of a DDFV scheme used for the discretization of a diffusion operator.

One example of the use of (56) is given in [7].

Notice that this approach to discretization of reaction terms is particularly natural if the DDFV scheme is viewed as a "gradient scheme" in the sense of Eymard, Guichard and Herbin [46], because in this case the unknown discrete solution in the scheme is approximated via a lifted function that, in our case, takes precisely the form $\frac{1}{3} w^{\mathfrak{M}^{o}}+\frac{2}{3} w^{\mathfrak{M}^{*}}$.

Remark 6. Note that, instead of adding the penalization operator in the scheme (42), one could treat the time evolution term in (42) using the discretization of type (56) for $b(u)$. 


\section{D. Appendix C: The coordination-decomposition algorithm}

The goal is to solve the nonlinear system

$$
b\left(u^{\mathfrak{T}, n}\right)-\Delta t \operatorname{div}^{\mathfrak{T}} \varphi\left(\nabla^{\mathfrak{T}} u^{\overline{\mathfrak{s}}, n}\right)=b\left(u^{\mathfrak{T}, n-1}\right)+\Delta t f^{\mathfrak{T}, n}
$$

(in the numerical tests, we drop the penalization operator needed for the theoretical justification of convergence of the scheme); recall that $u^{\overline{\mathfrak{s}}, n} \in R_{0}^{\overline{\mathbf{s}}}$, i.e., the boundary values of $u^{\overline{\mathfrak{s}}, n}$ are set to be zero. The algorithm, which is a simplified version of the one of [20], follows the guidelines of Glowinski and Marrocco $[53,54]$. Note that a convergence analysis of such an algorithm can be found in [20], for the more involved case of m-DDFV schemes.

We fix two parameters: $r>0$ and $\gamma>0$, and a tolerance threshold tol.

\section{Initialization of the algorithm}

- $k=0$

- $u_{0}^{\mathfrak{z}}=u^{\mathfrak{T}, n-1}$ (in the case $b$ is not invertible, one may pick $u_{0}^{\mathfrak{\tau}}=(b+\varepsilon I)^{-1}\left(b^{\mathfrak{T}, 0}\right)$ )

- errit $=1$

- $\operatorname{source}=b\left(u^{\mathfrak{T}, n-1}\right)+\Delta t f^{\mathfrak{T}, n}$

- $g_{0}^{\mathfrak{T}}=\nabla^{\mathfrak{T}} u_{0}^{\overline{\mathfrak{T}}}$

- $\lambda_{0}^{\mathfrak{T}}=-\varphi\left(\nabla^{\mathfrak{T}} u_{0}^{\overline{\mathfrak{\tau}}}\right)$.

While $($ errit $>t o l)$ do iterations of the algorithm $\left(u_{k-1}^{\mathfrak{T}}, g_{k-1}^{\mathfrak{T}}, \lambda_{k-1}^{\mathfrak{T}}\right) \rightarrow\left(u_{k}^{\mathfrak{T}}, g_{k}^{\mathfrak{T}}, \lambda_{k}^{\mathfrak{T}}\right)$

- First step Evaluation of $u_{k}^{\overline{\mathfrak{s}}} \in \mathbb{R}_{0}^{\overline{\mathfrak{s}}}$ solution of

$$
b\left(u_{k}^{\mathfrak{T}}\right)-\Delta t \operatorname{div}^{\mathfrak{T}}\left(r\left(\nabla^{\mathfrak{T}} u_{k}^{\overline{\mathfrak{T}}}-g_{k-1}^{\mathfrak{T}}\right)-\lambda_{k-1}^{\mathfrak{T}}\right)=\text { source }
$$

or, equivalently, of

$$
b\left(u_{k}^{\mathfrak{T}}\right)-r \Delta t \operatorname{div}^{\mathfrak{T}}\left(\nabla^{\mathfrak{T}} u_{k}^{\overline{\mathfrak{T}}}\right)=-\Delta t \operatorname{div}^{\mathfrak{T}}\left(r g_{k-1}^{\mathfrak{T}}+\lambda_{k-1}^{\mathfrak{T}}\right)+\text { source }
$$

(we can denote by source $_{k}$ the right-hand side of the above expression).

Thus we use the Newton method to solve this system; notice that it takes the form

$$
b\left(u_{k}^{\mathfrak{T}}\right)+r \Delta t A_{0} u_{k}^{\mathfrak{T}}=\text { source }_{k}
$$

where $A_{0}$ is the matrix corresponding to the CeVe-DDFV discretization of the linear $-\Delta$ operator $^{10}$ on the mesh $\boldsymbol{T}$. For the sake of completeness, we write the algorithm:

- Initialization : $X^{0}=u_{k-1}^{\mathfrak{T}}$

- While $\| b\left(X^{l}\right)+r \Delta t A_{0} X^{l}-$ source $_{k} \|>$ tol do

$$
X^{l+1}=X^{l}-\left(\operatorname{diag} b^{\prime}\left(X^{l}\right)+r \Delta t A_{0}\right)^{-1}\left(b\left(X^{l}\right)+r \Delta t A_{0} X^{l}-\text { source }_{k}\right)
$$

where diag · is the diagonal matrix with the prescribed vector · of diagonal entries.

\footnotetext{
${ }^{10}$ In the case of non-homogeneous Dirichlet boundary condition, we still take the zero conditions for the discrete Laplace operator, and the discrete boundary condition contributes to the source term of (57))
} 
$-u_{k}^{\mathfrak{s}}$ is assigned to be the final value of $X^{l}$.

- Second step Evaluation of $g_{k}^{\mathfrak{T}}=\left(g_{D, k}\right)_{D} \in\left(\mathbb{R}^{\mathfrak{D}}\right)^{3}$.

Here we have $\operatorname{card}(\mathfrak{D})$ decoupled nonlinear problems in $\mathbb{R}^{3}$ to be solved. In every diamond $D$ we have to solve the following problem:

$$
\varphi\left(g_{D, k}\right)+\lambda_{D, k-1}+r\left(g_{D, k}-\nabla_{D} u_{k}^{\overline{\bar{\tau}}}\right)=0
$$

Once more, we use the Newton method:

- Initialization : $Y^{0}=g_{D, k-1}$

- While $\left\|\varphi\left(Y^{l}\right)+\lambda_{D, k-1}+r\left(Y^{l}-\nabla_{D} u_{k}^{\bar{\tau}}\right)\right\|>$ tol do

$$
Y^{l+1}=Y^{l}-\left(D \varphi\left(Y^{l}\right)+r I_{3}\right)^{-1}\left(\varphi\left(Y^{l}\right)+\lambda_{D, k-1}+r\left(Y^{l}-\nabla_{D} u_{k}^{\bar{\tau}}\right)\right)
$$

where $I_{3}$ is the $3 \times 3$ identity matrix, and $D \varphi$ denotes the jacobian matrix of $\varphi: \mathbb{R}^{3} \rightarrow \mathbb{R}^{3}$. Notice that this $3 \times 3$ system can be solved manually, offline.

$-g_{D, k}$ is assigned to be the final value of $Y^{l}$.

- Third step Evaluation of $\lambda_{k}^{\mathfrak{T}} \in\left(\mathbb{R}^{\mathfrak{D}}\right)^{3}$.

$$
\lambda_{k}^{\mathfrak{T}}=\lambda_{k-1}^{\mathfrak{T}}+r \gamma\left(g_{k}^{\mathfrak{T}}-\nabla^{\mathfrak{T}} u_{k}^{\overline{\mathfrak{T}}}\right)
$$

\section{- Update of the stopping criterion.}

$$
\text { errit }=\| b\left(u_{k}^{\mathfrak{T}}\right)-\Delta t \operatorname{div}^{\mathfrak{T}} \varphi\left(\nabla^{\mathfrak{T}} u_{k}^{\overline{\mathfrak{T}}}\right)-\text { source } \| .
$$

\section{End of $k^{\prime}$ th iteration}

- At the end of the iterative procedure, assign $u^{\mathfrak{\tau}, n}$ to be the final value $u_{k}^{\mathfrak{\tau}}$.

\section{References}

[1] H.W. Alt and S. Luckhaus. Quasilinear elliptic-parabolic differential equations. Mat. Z. 183(1983), pp. 311-341.

[2] B. Andreianov. Time compactness tools for discretized evolution equations and applications to degenerate parabolic PDEs. In Proceedings of Finite Volumes for Complex Applications VI in Prague, pp.21-29. Springer Proc. Math., 4, Springer, Heidelberg, 2011.

[3] B. Andreianov, M. Bendahmane, F. Hubert and S. Krell. On 3D DDFV discretization of gradient and divergence operators. I. Meshing, operators and discrete duality. IMA J. Numer. Anal., 32(4):1574-1603, 2012.

[4] B. Andreianov, M. Bendahmane, and K.H. Karlsen. Discrete duality finite volume schemes for doubly nonlinear degenerate hyperbolic-parabolic equations. J. Hyp. Diff. Equ. 7(1):1-67, 2010.

[5] B. Andreianov, M. Bendahmane and K.H. Karlsen. A gradient reconstruction formula for finite volume schemes and discrete duality. In R. Eymard and J.-M. Hérard, editors, Finite Volume For Complex Applications, Problems And Perspectives. 5th International Conference, 161-168. London (UK) Wiley, 2008. 
[6] B. Andreianov, M. Bendahmane, K.H. Karlsen and S. Ouaro. Structural stability for variable exponent elliptic problems. II. The p(u)-Laplacian and coupled problems. Nonlinear Anal. Ser. A, 72(12):46494660, 2010.

[7] B. Andreianov, M. Bendahmane, K.H. Karlsen and Ch. Pierre. Convergence of discrete duality finite volume schemes for the cardiac bidomain model. Netw. Heterog. Media 6(2):195-240, 2011.

[8] B. Andreianov, M. Bendahmane and R. Ruiz Baier. Analysis of a finite volume method to solve a cross-diffusion population system. M3AS Math. Models Meth. Appl. Sci. 21(2):307-344, 2011.

[9] B. Andreianov, F. Boyer, and F. Hubert. Finite volume schemes for the $p$-Laplacian on Cartesian meshes. M2AN Math. Model. Numer. Anal. 38(6):931-959, 2004.

[10] B. Andreianov, F. Boyer and F. Hubert. Besov regularity and new error estimates for finite volume approximations of the p-laplacian. Numer. Math., 100(4):565-592, 2005.

[11] B. Andreianov, F. Boyer and F. Hubert. On finite volume approximation of regular solutions of the p-laplacian. IMA J.Numer. Anal., 26(3):472-502, 2006.

[12] B. Andreianov, F. Boyer, and F. Hubert. Discrete duality finite volume schemes for Leray-Lions type elliptic problems on general 2D meshes. Numer. Meth. PDE, 23(1):145-195, 2007.

[13] B. Andreianov, M. Gutnic, and P. Wittbold. Convergence of finite volume approximations for a nonlinear elliptic-parabolic problem: a "continuous" approach. SIAM J. Numer. Anal. 42(1):228-251, 2004.

[14] B. Andreianov, F. Hubert and S. Krell. Benchmark 3D: a version of the DDFV scheme with cell/vertex unknowns on general meshes. In Proceedings of Finite Volumes for Complex Applications VI in Prague, pp.937-948. Springer Proc. Math., 4, Springer, Heidelberg, 2011.

[15] B. Andreianov and P. Wittbold. Convergence of approximate solutions to an ellipticparabolic equation without the structure condition. NoDEA Nonlin. Differ. Equ. Appl. 19:695-717, 2012.

[16] M. Bendahmane and K.H. Karlsen. Analysis of a class of degenerate reaction-diffusion systems and the bidomain model of cardiac tissue. Netw. Heterog. Media 1:185-218, 2006.

[17] M. Bendahmane and K.H. Karlsen. Convergence of a finite volume scheme for the bidomain model of cardiac tissue. Appl. Numer. Math., 59(9):2266-2284, 2009.

[18] Ph. Bénilan and P. Wittbold. Sur un problème parabolique-elliptique. (French) M2AN Math. Model. Num. Anal. 33(1):121-127, 1999.

[19] C. Berthon, Y. Coudière and V. Desveaux. Development of DDFV methods for the Euler equations. In Proceedings of Finite Volumes for Complex Applications VI in Prague, pp.117-124. Springer Proc. Math., 4, Springer, Heidelberg, 2011

[20] F. Boyer and F. Hubert. Finite volume method for 2D linear and nonlinear elliptic problems with discontinuities. SIAM J. Num. Anal. 46(6):3032-3070, 2008.

[21] F. Boyer, F. Hubert and S. Krell. Non-overlapping Schwarz algorithm for solving 2D-mDDFV schemes. IMA J. Num. Anal. 30(4):1062-1100, 2010.

[22] M. Bessemoulin-Chatard, C. Chainais-Hillairet, F. Filbet. On discrete functional inequalities for some finite volume schemes. HAL preprint http://hal.archives-ouvertes.fr/hal-00672591

[23] C. Chainais-Hillairet, S. Krell and A. Mouton. Study of discrete duality finite volume schemes for the Peaceman model. HAL preprint http://hal.archives-ouvertes.fr/hal-00790449

[24] Y. Coudière, Th. Gallouët and R. Herbin. Discrete Sobolev inequalities and $L^{p}$ error estimates for finite volume solutions of convection diffusion equations. M2AN Math. Model. Numer. Anal. 35(4) (2001) 767-778.

[25] Y. Coudière and F. Hubert. A 3D Discrete Duality Finite Volume method for nonlinear elliptic equations. In: A. Handlovičovà, P. Frolkovič, K. Mikula, D. Ševčovič (Eds.), Proceedings of Algoritmy 2009, pp. 51-60, 2009.

[26] Y. Coudière and F. Hubert. A 3D discrete duality finite volume method for nonlinear elliptic equation. SIAM J. Sci. Computing 33(4):1739-1764, 2011.

[27] Y. Coudière, F. Hubert and G. Manzini. Benchmark 3D: CeVeFE-DDFV, a discrete duality scheme with cell/vertex/face+edge unknowns. In Proceedings of Finite Volumes for Complex Applications VI in Prague, pp.177-184. Springer Proc. Math., 4, Springer, Heidelberg, 2011.

[28] Y. Coudière and G. Manzini. The Discrete Duality Finite Volume Method for Convection-diffusion Problems SIAM J. Numer. Anal. Volume 47(6):4163-4192, 2010. 
[29] Y. Coudière and Ch. Pierre. Benchmark 3D: CeVe-DDFV, a discrete duality scheme with cell/vertex unknowns. In Proceedings of Finite Volumes for Complex Applications VI in Prague, pp.234-254. Springer Proc. Math., 4, Springer, Heidelberg, 2011.

[30] Y. Coudière, Ch. Pierre and R. Turpault. Solving the fully coupled heart and torso problems of electrocardiology with a 3D discrete duality finite volume method. Preprint HAL 2009, http://hal.archivesouvertes.fr/hal-00016825

[31] Y. Coudière, Ch. Pierre, O. Rousseau and R. Turpault. 2D/3D discrete duality finite volume scheme. Application to ECG simulation. Int. J. Finite Vol. 6(1):1-24, 2009.

[32] Y. Coudière, Ch. Pierre, O. Rousseau and R. Turpault. 2D/3D discrete duality finite volume scheme (DDFV) applied to ECG simulation. In R. Eymard and J.-M. Hérard, editors, Finite Volume For Complex Applications, Problems And Perspectives. 5th International Conference, 313-320. London (UK) Wiley, 2008.

[33] Y. Coudière, J.-P. Vila, Ph. Villedieu. Convergence rate of a finite volume scheme for a two-dimensional convection-diffusion problem. M2AN Math. Model. Numer. Anal. 33(3):493-516, 1999.

[34] S. Delcourte. Développement de méthodes de volumes finis pour la mécanique de fluides. Ph.D. thesis, Univ. Paul Sabatier, Toulouse, 2007.

[35] K. Domelevo, S. Delcourte and P. Omnes. A discrete duality finite volume approach to Hodge decomposition and div-curl problems on almost arbitrary two-dimensional meshes. SIAM J. Numer. Anal. 45(3):1142-1174, 2007.

[36] K. Domelevo and P. Omnès. A finite volume method for the Laplace equation on almost arbitrary two-dimensional grids. M2AN Math. Model. Numer. Anal. 39(6):1203-1249, 2005.

[37] J. Droniou. Finite volume approximations for fully nonlinear elliptic equations in divergence form. M2AN Math. Model. Numer. Anal. 40(6):1069-1100, 2006.

[38] J. Droniou. Finite volume methods for diffusion equations: introduction to and review of different methods. Preprint submitted to M3AS Math. Models Meth. Appl. Sci., 2013.

[39] J. Droniou, T. Gallouët and R. Herbin. A finite volume scheme for a noncoercive elliptic equation with measure data. SIAM J. Numer. Anal. 41, no. 6, 1997-2031, 2003.

[40] R. Eymard, T. Gallouët, M. Gutnic, R. Herbin and D. Hilhorst. Approximation by the finite volume method of an elliptic-parabolic equation arising in environmental studies. M3AS Math. Models Meth. Appl. Sci. 11(9):1505-1528, 2001

[41] R. Eymard, T. Gallouët, and R. Herbin. Finite Volume Methods. Handbook of Numerical Analysis, Vol. VII, P. Ciarlet, J.-L. Lions, eds., North-Holland, 2000.

[42] R. Eymard, T. Gallouët, and R. Herbin. Discretisation of heterogeneous and anisotropic diffusion problems on general non-conforming meshes. SUSHI: a scheme using stabilisation and hybrid interfaces, IMA J. Numer. Anal. 30(4)1009-1043, 2010.

[43] R. Eymard, T. Gallouët, and R. Herbin. Cell centered discretisation of non linear elliptic problems on general multidimensional polyhedral grids. J. Numer. Math. 17(3):173-193, 2009.

[44] R. Eymard, T. Gallouët, R. Herbin and A. Michel. Convergence of a finite volume scheme for nonlinear degenerate parabolic equations. Numer. Math. 92(1):41-82, 2002.

[45] R. Eymard, G. Henry, R. Herbin, F. Hubert, R. Klöfkorn, and G. Manzini, 3D Benchmark on discretization schemes for anisotropic diffusion problems on general grids, In Proc. of Finite Volumes for Complex Applications VI in Prague, pp.95-130. Springer Proc. Math., 4, Springer, Heidelberg, 2011.

[46] R. Eymard, C. Guichard and R. Herbin. Small-stencil 3D schemes for diffusive flows in porous media. M2AN, 46:265-290, 2012.

[47] R. Eymard, M. Gutnic and D. Hilhorst. The finite volume method for Richards equation. Comput. Geosci. 3:259-294, 1999.

[48] R. Eymard, A. Handlovičová and K. Mikula. Approximation of nonlinear parabolic equations using a family of conformal and non-conformal schemes. Commun. Pure Appl. Anal. 11(1):147-172, 2012.

[49] F. Filbet. A finite volume scheme for the Patlak-Keller-Segel chemotaxis model. Numer. Math. 104:457$488,2006$.

[50] T. Gallouët and R. Herbin. Finite volume approximation of elliptic problems with irregular data. In: Finite volumes for complex applications. Problems and Perspectives. II, F. Benkhaldoun, M. Hänel and R. Vilsmeier eds, Hermès, 155-162, 1999. 
[51] T. Gallouët and J.-C. Latché. Compactness of discrete approximate solutions to parabolic pdes - application to a turbulence model. Comm. Pure Appl. Anal. 11(6),2012.

[52] A. Glitzky and J.A. Griepentrog. Discrete Sobolev-Poincare inequalities for Voronoï finite volume approximations. SIAM J. Numer. Anal. 48(1):372-391, 2010.

[53] R. Glowinski and A. Marrocco. Sur lapproximation, par éléments finis dordre un, et la résolution, par pénalisation-dualité, dune classe de problèmes de Dirichlet non linéaires. RAIRO Rev. Française Automat. Informat. Recherche Opérationnelle, 9(R-2):41-76, 1975.

[54] R. Glowinski. Numerical methods for nonlinear variational problems. Scientific Computation. SpringerVerlag, Berlin, 1984, 2008.

[55] R. Herbin and F. Hubert. Benchmark on discretization schemes for anisotropic diffusion problems on general grids. In R. Eymard and J.-M. Hérard, editors, Finite Volume For Complex Applications, Problems And Perspectives. 5th International Conference, 659-692. London (UK) Wiley, 2008.

[56] F. Hermeline. Une méthode de volumes finis pour les équations elliptiques du second ordre. (French) C. R. Math. Acad. Sci. Paris 326(12):1433-1436, 1998.

[57] F. Hermeline. A finite volume method for the approximation of diffusion operators on distorted meshes. J. Comput. Phys. 160(2):481-499, 2000.

[58] F. Hermeline. A finite volume method for solving Maxwell equations in inhomogeneous media on arbitrary meshes. C. R. Math. Acad. Sci. Paris 339(12):893-898, 2004.

[59] F. Hermeline. Approximation of 2D and 3D diffusion operators with discontinuous full-tensor coefficients on arbitrary meshes. Comput. Methods Appl. Mech. Engrg., 196(21-24):2497-2526, 2007.

[60] F. Hermeline. A finite volume method for approximating 3D diffusion operators on general meshes. $J$. Comput. Phys. 228(16):5763-5786, 2009.

[61] D. Kotorová. Discrete Duality Finite Volume scheme for the curvature-driven level set equation. Acta Polytechnica Hungarica 8(3):7-12, 2011.

[62] S. Krell. Schémas Volumes Finis en mécanique des fluides complexes. (French) Ph.D. Thesis, Univ. de Provence, Marseilles, 2010.

[63] S. Krell. Stabilized DDFV schemes for Stokes problem with variable viscosity on general 2D meshes. Num. Meth. PDEs 27(6):1666-1706, 2011.

[64] S. Krell and G. Manzini. The Discrete Duality Finite Volume method for the Stokes equations on 3D polyhedral meshes. SIAM J. Numer. Anal. 50(2):808-837, 2012.

[65] S.N. Kruzhkov. Results on the nature of the continuity of solutions of parabolic equations and some of their applications. Mat. Zametki 6(1):97-108, 1969; english tr. in Math. Notes 6(1):517-523, 1969.

[66] A. H. Le, P. Omnès. Discrete Poincaré inequalities for arbitrary meshes in the discrete duality finite volume context. HAL-CEA preprint http://hal-cea.archives-ouvertes.fr/cea-00726543

[67] A. H. Le, P. Omnès. An a Posteriori Error Estimation for the Discrete Duality Finite Volume Discretization of the Stokes Equations. HAL-CEA preprint http://hal-cea.archives-ouvertes.fr/cea-00726531

[68] B. Martin and F. Pascal. Discrete duality finite volume method applied to linear elasticity. In Proceedings of Finite Volumes for Complex Applications VI in Prague, pp.663-671. Springer Proc. Math., 4, Springer, Heidelberg, 2011.

[69] R.A. Nicolaides. Direct discretization of planar div-curl problems. SIAM J. Numer. Anal., 29:32-56, 1992.

[70] P. Omnès, Y. Penel and Y. Rosenbaum. A Posteriori Error Estimation for the Discrete Duality Finite Volume Discretization of the Laplace Equation. SIAM J. Numer. Anal. 47(4):2782-2807, 2009.

[71] F. Otto. $L^{1}$ contraction and uniqueness for quasilinear elliptic-parabolic equations. J. Diff. Eq., 131:20$38,1996$.

[72] Ch. Pierre. Modélisation et simulation de l'activité électrique du coeur dans le thorax, analyse numérique et méthodes de volumes finis. (French) Ph.D. Thesis, Université de Nantes, 2005. 